UNEXPOSED, UNEXPLORED, AND UNRESOLVED CONFLICT AS PRECURSORS TO THE FAILURE OF HIGH TECHNOLOGY PROJECTS

A Dissertation
Presented to
The Faculty of the Graduate School
University of Missouri-Columbia
In Partial Fulfillment
Of the Requirements for the Degree
Doctor of Philosophy
William Allan Reed
Dr. Michael Kramer, Dissertation Supervisor

May 2006 
The undersigned, appointed by the Dean of the Graduate School, Have examined the dissertation entitled.

\section{UNEXPOSED, UNEXPLORED, AND UNRESOLVED CONFLICT AS PRECURSORS TO THE FAILURE OF HIGH TECHNOLOGY PROJECTS}

Presented by William A. Reed

A candidate for the degree of Doctor of Philosophy

And hereby certify that in their opinion it is worthy of acceptance. 
Dedicated to my dad

\section{Glenn Harold Reed}

who gave me this opportunity 


\section{ACKNOWLEDGEMENTS}

This project represents a life milestone that was only possible with the support of those who believed in me and were gracious enough to endure through the months when my abilities to see outside of my work were so limited. I am truly indebted to those individuals.

My doctoral journey began when my daughter encouraged me to explore new possibilities and then shared many of the small steps with me along the way. Because of her maturity and self-reliance, I was able to turn my attention to this grand adventure. She continues to be my major inspiration and is clearly destined to achieve that which she strives for. The ongoing support of my immediate and extended family were also central to getting through each day when there was little time to conduct a normal life outside of the academy.

I join many others who have been profoundly humbled by their doctoral education, being continually reminded that there are many teachers in life and much to learn. I was very fortunate to have had Dr. Kramer as an advisor who supported and encouraged me from my first inquiry about the program through the final draft of this dissertation. Dr. Kramer was always available for consultation and provided consistent detailed feedback, which were key factors in my academic growth. In terms of teaching, there are few professors that can reach Dr. Kramer’s level, and I'm sure there is no better teacher of quantitative methods. I also was very fortunate to have strong scholars and encouraging teachers for my committee members. Dr. Dougherty earned my respect from the first day of classes when she assigned more work than I thought possible, but never wasted my time. She crafted every reading assignment, project, and discussion to challenge me with new ideas and concepts. And she often could see more potential in her students then we saw in ourselves. Dr. McKinney taught me more about philosophy than I would have ever expected to know. His classes were always over too soon, which speaks highly of his 
ability to focus obscure concepts and make them interesting and relevant to his students' own research programs. My program would have also been incomplete without Dr. Hess who taught me how to teach in the college environment and provided encouragement at every step of my program. His expert knowledge in the interpersonal arena was often helpful and he frequently volunteered articles from his own research library. Dr. Turban was my outside advisor who was invaluable in helping me develop a more robust understanding of current management literature and perspectives, especially in regard to the assessment of qualitative and quantitative research data in business applications. His energy and willingness to help students outside of his department was much appreciated.

Finally, while there is little time for friendships during a doctoral program, I am thankful for having many friends and colleagues who would find time for an occasional lunch or coffee break and provide encouragement and support during my many weekends and late nights of study. 


\section{TABLE OF CONTENTS}

ACKNOWLEDGEMENTS ............................................... ii

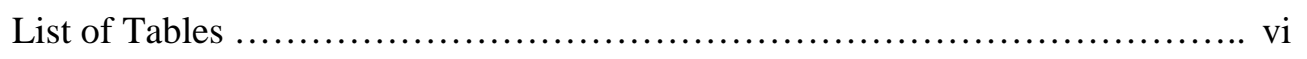

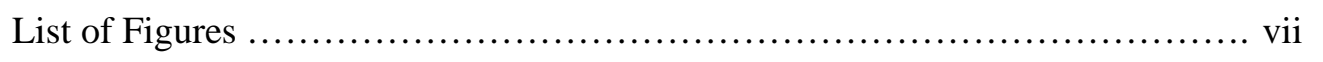

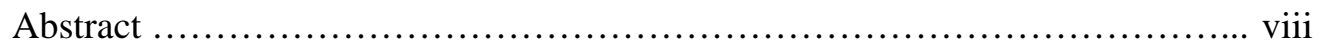

Chapter

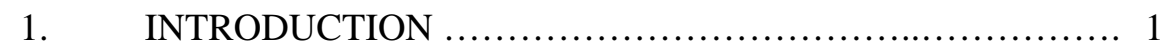

2. REVIEW OF LITERATURE .............................. 12

Research questions .................................. 66

3. METHODOLOGY .................................... 69

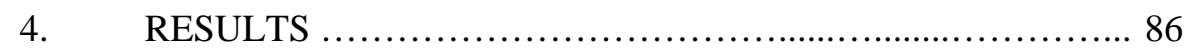

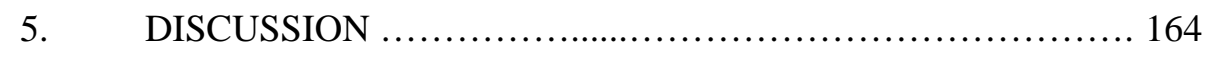

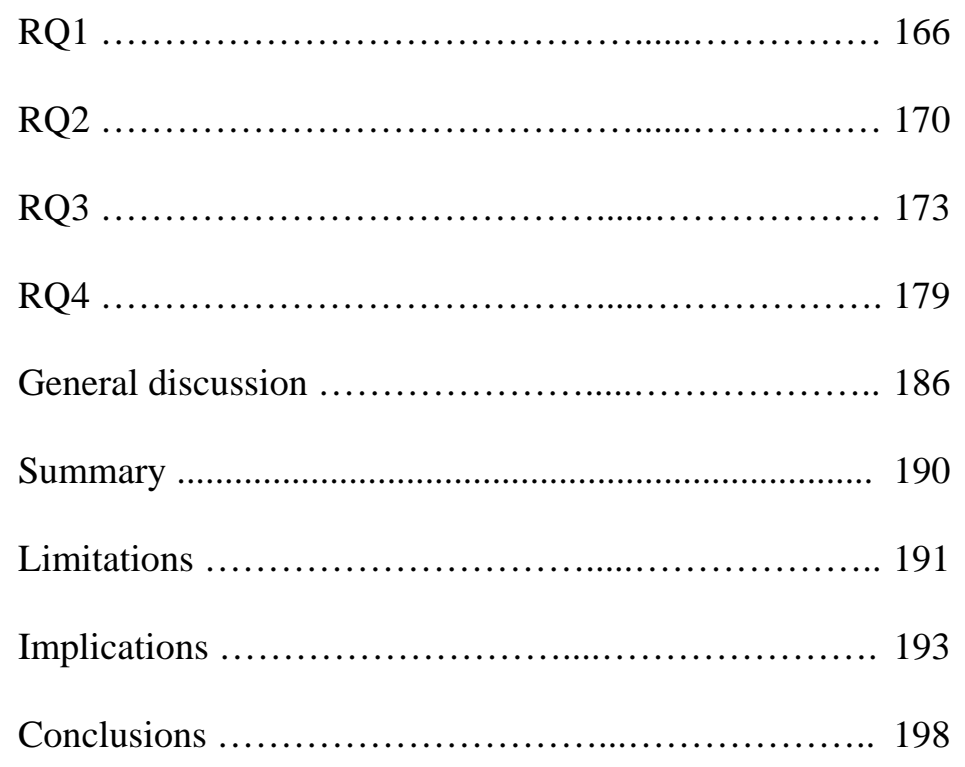

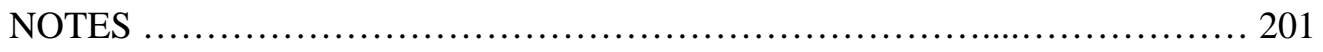

APPENDIX

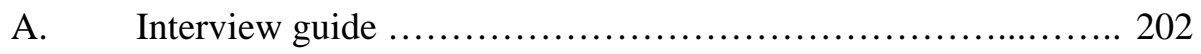




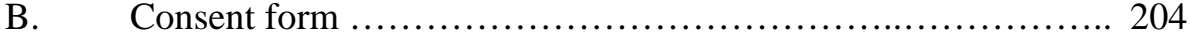

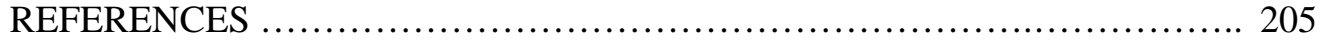

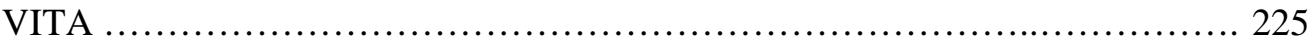




\section{LIST OF TABLES}

Table

Page

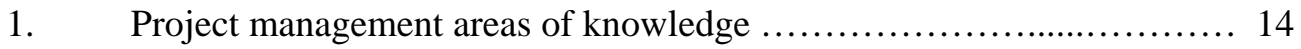

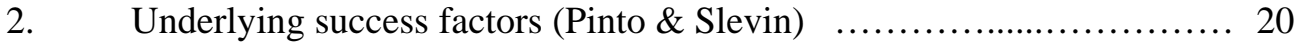

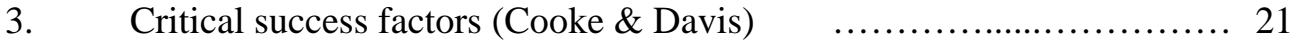

4. Decision errors (McCray, Purvis, \& McCray) ........................ 24

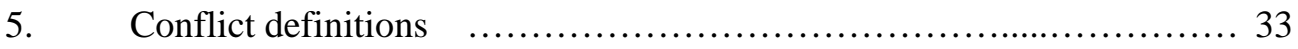

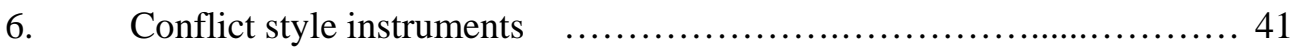

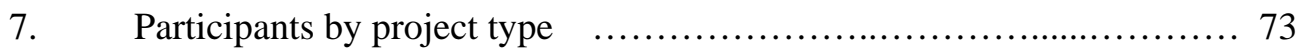

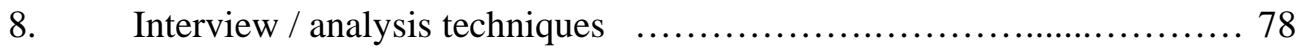

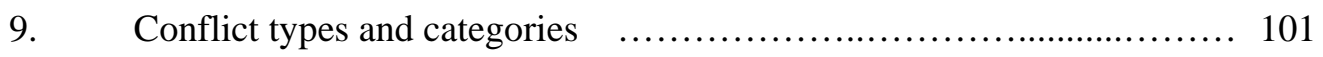

10. Sensemaking variations by conflict type ............................ 185 


\section{LIST OF FIGURES}

Figure

Page

1. $\quad$ Blake \& Mouton grid .............................................. 36

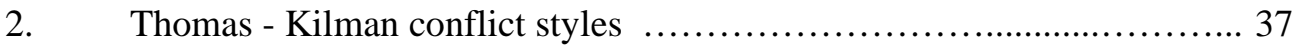




\begin{abstract}
This qualitative study of high-technology projects investigated how enduring conflict can operate as a precursor to project failure. Three types of conflict were found to be indicative to the outcome of projects. Unexposed conflict represented problems with obtaining knowledge and coherency for important project-related differences. Unexplored conflict reflected issues with power and voice, where important communication about known differences was consistently inhibited or suppressed. Finally, unresolved conflict centered on issues of timing and action which involved difficulties with adapting to change, negotiating solutions to problems, and agreeing on priorities and scope. Two contextual factors, project complexity and the ambiguity of success provided a substantial contribution to the experience of these conflicts among the participants. The theoretical construct of sensemaking (Weick, 1995) provided a framework to understand how each type of conflict was constructed and had the potential to become enduring. Implications are discussed with emphasis on the potential for qualitative assessments to provide important insights into the status of complex high technology projects.
\end{abstract}




\section{UNEXPOSED, UNEXPLORED, AND UNRESOLVED CONFLICT AS PRECURSORS}

TO THE FAILURE OF HIGH TECHNOLOGY PROJECTS

\section{CHAPTER 1}

High technology products and services have increasingly been developed and implemented through formal project teams requiring intensive cross-functional collaboration and coordination with multiple stakeholders. In addition, the emergence of rapidly changing technology and increasingly competitive pressures have resulted in exceptionally complex and risk-laden projects being pursued under extremely challenging time and cost constraints. Many of these projects fail.

In 1987, Marriott, Hilton, and Budget Rent-a-Car entered into a joint venture with AMRIS [AMR Information Services] to develop a state-of-the-art $\$ 55.7$ million dollar computerized reservation system called CONFIRM that would allow consumers to make airline, hotel, and car rental reservations through a single system. At the end of April 1992, the AMRIS chairperson wrote the three partners saying: "Unfortunately, things have not gone as planned ... the individuals whom we gave responsibility for managing CONFIRM ... have apparently deliberately concealed a number of important technical and performance problems.” On May 1, 1992, AMRIS’ vice-chairperson circulated a letter internally stating that "some people who have been part of CONFIRM'S management did not disclose the true status of the project in a timely manner. This has created more difficult problems - of both business ethics and finance than would have existed if those people had come forward with accurate information.” In July 1992, after spending 3.5 years and \$125 million on CONFIRM, the project was canceled (Smith, Keil, \& Depledge, 2001, p. 190) citing (Oz, 1994).

This is not a rare event. Failed projects in the United States have been estimated to cost industry in excess of \$145 billon per year (Field, 1997). In the information technology (IT) arena where most work is performed through the formal project management approach (Sauer, Liu, \& Johnston, 2001), some estimates have suggested that less than 25 percent of IT projects meet all of their cost, schedule, and functionality targets (Martin \& Chan, 1996). Similarly, an industry survey reports that only 26 percent of software projects were completed on time and on budget, while 28 percent were canceled and the remaining 46 percent were over time and budget with 
fewer features than promised (Standish Group International, 1999). In speaking about the ongoing problems with IT projects, Lyytinen and Robey (1999) exhort that IT groups have not just failed to learn, but that they have "learned to fail." This culture has created projects that are routinely over budget, behind schedule, and implemented with fewer features than promised (Gibbs, 1994). This has also become a global issue. For example, a KPMG survey of 120 organizations in the UK found that 62 percent had experienced similar problems (KPMG, 1995).

Reports of major project failures abound from numerous organizational entities. In an example from the insurance industry, the project failure was more implementation related than technical. The Canadian life insurance company Integra (a pseudonym) developed the Banking and Loan Insurance Software System (BLISS) to expand their loan business operations by providing the software free of charge to credit unions in exchange for exclusive marketing rights (Roy \& Aubert, 2003). In partnership with an IT consulting firm (INTEX, also a pseudonym) known for their expertise in the banking industry, Integra launched the project in December 1995. Although the project was organized around a formal structure that included a steering committee, list of work requirements, prototypes, and user groups, it quickly ran into a number of problems. These included a more complex than anticipated integration of legacy file systems, insufficient computing power of the workstations, and a lack of credit union participation at critical points in the development cycle. However, the project proceeded until the software was ready to demonstrate to the end user clients.

The first clear sign that something was seriously amiss with the project materialized when, in September 1996, the marketing team returned from a field trip empty-handed. Although the Credit Unions’ managers generally expressed a genuine interest in the new system, they seemed somewhat reluctant to commit themselves in any meaningful way to the project. This initial disquieting perception crystallized when, a few weeks later, at the end of the development phase, the project managers tried to enlist pilot sites to field-test the new system. As reported by Jim Cochran, the project seemed to hit a brick wall: "How many do you think that I [have] sold? I have ... even when we offered them the system for free, not a single one wanted to become a test site, not even those that had participated in the User Group. They didn't want any part of it!" (Roy \& Aubert, 2003, p. 106). 
Ultimately, “Integra’s management canceled the project, and negotiated an acceptable settlement with Intex Consulting to put an end to the development effort. In reviewing the project, the participants were perceived to have made rational decisions and the CEO of the organization was not able to assign responsibility for the costly outcome (Roy \& Aubert, 2003).

Perhaps the most widely known high technology project failure involved the baggage handling system at the Denver International Airport (DIA). Because DIA was designed to be the United States largest airport, the operation of the baggage handling system was critical. BAE Automated Systems was contracted to develop an integrated computer-based system that would be substantially more efficient than similar systems. “An information system composed of 55 networked computers, 5,000 electric eyes, 400 radio frequency receivers, and 56 bar code scanners was to orchestrate the safe and timely arrival of every suitcase and ski bag at DIA” (Montealegre \& Keil, 2000, p. 418). It was the failure of this very system that prevented the entire airport from opening in October, 1993. The first public indication of a critical problem in the DIA baggage system occurred in late April, 1994 when reporters were invited to observe a test of 7000 bags that were to be moved to Continental's Concourse A and United's Concourse B. After reporters noticed clothing and personal items strewn beneath the tracks of the baggage cars the testing was halted (Montealegre \& Keil, 2000). Further investigation of the operational problems with the system found them to be pervasive and enduring. When DIA finally opened in February 1995 it was close to \$2 billion over budget. Even then, it opened with two concourses operating with a manual baggage handling system.

\section{Formal Project Management}

Increasingly, the response to the high rate of project failures among high technology projects has been to emphasize and strengthen formal project management practices (Ives, 2005). This has been institutionalized by the establishment of professional organizations, official journals, standards, training, and certification. More recently, those who systematically perform project management have been identified as part of a developing profession (Kenny, 2003; Wang, 
2003), although some have questioned its overall acceptance and legitimacy (Urli \& Urli, 2000). However, at present (2006), membership in the Project Management Institute (PMI) is reported to exceed 150,000 members (PMI, 2005b) and PMI certification has become a common requirement for those employed as project managers in the United States.

The Project Management Institute compiles a collection of information about project management practices and publishes a guide described as a "body of knowledge” (PMBOK ${ }^{\circledR}$ Guide $^{1}$ ) to disseminate best practices to its members. The PMBOK defines a standard project management framework to include 37 component processes (PMI, 2005a), which include categories such as planning tools, cost estimation, risk analysis, and control techniques that are general enough to be applied to "most projects most of the time” (PMI, 2005a, p. 3). These processes were obtained from the practices of highly diverse firms resulting in what some have called more of a "heterogeneous toolbox than a body of knowledge” (Urli \& Urli, 2000, p. 33). Nevertheless, this document has become highly influential in scripting and justifying project management practices. ${ }^{2}$

Complex high-technology projects have numerous sub-tasks, each with specific requirements, deliverables, capability demonstrations, and delivery dates. When the practitioners follow the PMBOK guidelines, the projects also include immense documentation, specific procedures, and well defined processes to coordinate and communicate activities. So with such formalized processes, why do high technology projects fail at such an alarming rate and so late in the project cycle?

Numerous scholars and consultants have weighed in on the reasons for project failure. Perhaps the most frequently cited reason is project escalation, where organizations remain committed to a failing course of action although little hope remains for its success (M. Keil, 1995; M. Keil \& Robey, 1999; M. Keil \& Robey, 2001; Montealegre \& Keil, 2000; Smith et al., 2001). However, many other causes have been claimed. Whittaker (1999) surveyed Canada’s 1,450 leading public and private institutions about IT project failure. The three most common reasons 
given were poor project planning, a weak business case, and lack of top management involvement and support. From this same study other reasons included schedule and budget overruns, unproven technology, poor estimates, and vendors’ inability to meet their commitments. Similarly, Field (1997) suggests that projects fail because organizations do not properly address the users' needs or adequately define the project’s scope. Pinto and Kharbanda (1996) take a broader perspective in identifying what they describe as twelve important contributing actions that promote project failure. These are:

1. Ignore the project environment (including stakeholders)

2. Push a new technology to market too quickly.

3. Don't bother building in fallback options.

4. When problems occur, shoot the one most visible.

5. Let new ideas starve to death from inertia.

6. Don’t bother conducting feasibility studies.

7. Never admit a project is a failure.

8. Over-manage project managers and their teams.

9. Never, never conduct post-failure reviews.

10. Never bother to understand project trade-offs.

11. Allow political expediency and infighting to dictate crucial project decisions. 12. Maker sure the project is run by a weak leader.

Pinto and Kharbanda (1996, p. 46)

While much of the heuristic advice is too abstract to provide specific guidance, it has often implicated management practices for failing to adequately control the project activities throughout the project. For example, Glaser (2005) argues that senior leadership often fails to respect uncertainty, under-nourish initiatives, anticipate short-term disruptions, value invisible progress, and disregard the stability of technology. Consequently, a significant emphasis has been placed on management strategies to avoid project failures. These have frequently been 
described in terms of heuristics and tips (Gardner, 2000) or in-depth approaches toward risk management (Royer, 2000). Risk is the general category for identified concerns that through experience or insight may lead to project failure. Therefore, risk management has been established in the PMBOK as a structured practice to be conducted throughout a formal project.

Risk management provides a systematic and often statistical approach toward estimating resource consumption and functional performance along with planning activities to neutralize the risks. However, the risk management approach has a number of limitations. First, risks have to be recognized to be addressed. Risks that are unknown or unexpected may affect the project at any phase of its course. Second, when risks are recognized, they still may have a high degree of uncertainty that makes them difficult to abate. In general, risks are addressed by implementing either a mitigation plan or a contingency plan (Royer, 2000). Either of these require additional resources and introduce their own complexity and risk to the project. Finally, risks may be hidden in unrecognized assumptions about the project and its stakeholders. These are difficult to surface because they are embedded in the organization's culture (Schein, 1992). However, certain risks seem to be universal. In a study of project managers from three different countries (Hong Kong, Finland, U.S.), there was a strong consensus for eleven risk factors in IT projects, which were:

1. Lack of top management commitment to the project

2. Failure to gain user commitment

3. Misunderstanding the requirements

4. Lack of adequate user involvement

5. Failure to meet end user expectations

6. Changing scope/objectives

7. Lack of required knowledge/skills in the proper personnel

8. Lack of frozen requirements

9. Introduction of new technology 


\section{Insufficient/inappropriate staffing}

\section{Conflict between user departments}

(M. Keil, Cule, Lyytinen, \& Schmidt, 1998, p. 78)

The researchers noted that only one of these factors involved technology and it was not particularly prominent when compared to the other risks. The study participants generally felt that the technical problems were controllable and could be accounted for in a project plan. However, the non-technical issues were the predominant risks because they were not perceived to be adequately controllable.

The common element in all of these "reasons" for project failure is that a potentially problematic issue must first be known and recognized as significant before it can be addressed by management. This assumes that project information has the same meaning across participants and becomes available when it is discovered by the project members. However, this is not always the case. Some have argued that evidence of a failing project frequently exists at the lower ranks of a project, yet often fails to move up the hierarchy (M. Keil \& Robey, 2001). A number of reasons have been posited for this. First, a mum effect has been described where individuals demonstrate a reluctance to share bad news on projects primarily because of the risk of feared consequences (Smith et al., 2001). The opposite of the mum effect is the act of whistle-blowing. While this idea of reporting the perceived wrongdoings of others might be officially rewarded, it is frequently not seen as legitimate activity in most organizations (Dozier \& Miceli, 1985; M. Keil \& Robey, 1999). That is, individuals are reluctant to share the known misdeeds of others for fear of being punished or ostracized (Weinstein, 1979). A third reported barrier in communicating negative project status has been described as the deaf effect. "By remaining deaf in the presence of trouble, actors may hope to avoid dealing with difficult problems. They may also remain deaf to disassociate themselves from a failing endeavor” (M. Keil \& Robey, 1999, p. 82). While this is routinely attributed to managers, other stakeholders may selectively become deaf to specific information that impinges on their own individual or group interests. Finally, 
project team members may deliberately misinform the leadership regarding project status (Sims, 1993). This may be described as a misinforming effect, and is perceived to occur for several reasons including lack of effort, timing, social niceties, self-defense, incompetence, and politicking. Eventually, information about failing projects can no longer be managed (suppressed) by these techniques and becomes a visible source of open conflict as illustrated by the press demonstration at the Denver airport.

The present study explores these and other aspects of project communication as both a complex and central issue relating to project success. Yet, this stands in sharp contrast with the PMBOK and other project management texts, which represents project communication as a basic exchange of information using a sender-receiver model (Axley, 1984). The consequence of accepting such a limited model of communication is to deemphasize its role in how project problems come to exist and influence outcomes. Thus, the purpose of the present study is to explore project communication issues in more detail and with a more complex model of communication. To that end, this study adopts an interpretive paradigm that frames project management as a socially constructed environment where individual stakeholders vie for their perspectives to prevail. The management of conflict then becomes a critical factor that has the potential to reveal important differences among the participants, surface underlying expectations, and identify sources of power, which may then be a significant indicator of project outcomes.

The existence of conflict in highly complex and ambiguous situations is explained by the theory of sensemaking (Weick, 1995), which suggests that individuals actively seek to reduce uncertainty by developing plausible explanations for their experiences (Volkema, Farquhar, \& Bergmann, 1996). High technology projects are expected to be an especially rich source of sensemaking because the complexity of technological development has been directly equated with ambiguity in the form of organizational equivoque (Weick, 2001). Weick defined an equivoque as "something that admits of several possible or plausible interpretations and therefore can be esoteric, subject to misunderstandings, uncertain, complex, and recondite” (p. 148). This 
is expected to occur throughout the stages of high technology projects, as each project phase represents attempts to accomplish novel solutions to engineering problems under restricted time, cost, quality, and performance constraints.

The constructionist perspective (Burr, 1995) adopted in this study provides the opportunity to explore how individuals uniquely and collectively make sense of their experiences in projects. The meanings developed for these experiences may be reflected by competing narratives among stakeholders as the participants become committed to their interpretations of the project situation. Therefore, access to these alternative stories can become an important source of information since they may represent the rationale for decisions and behaviors leading to eventual project failure. Thus, the definition of conflict developed in the current study evolves from a constructionist paradigm to suggest that conflict may be viewed as incongruent sensemaking about the project. That is, the interlocutors come into conflict as they develop incompatible meanings for important project issues. Three important considerations emerge from this approach. First, these alternate meanings may contain valuable information that could be used to identify problems earlier in the project than might otherwise occur, resulting in more successful projects. For example, situations like the mum effect and the deaf effect may be better understood and avoided if these alternative meanings for project situations are routinely given space for consideration. Second, if conflict as an incongruent system of meanings becomes enduring, it may exert a constitutive force, in the vein of a self-fulfilling prophesy. That is, as individuals become committed to a competing version of the project situation, they may be increasingly likely to use their discretionary power to act in service of those commitments (Weick, 1995). In that way, conflicting beliefs about the trajectory of the project may result in discordant actions that effectively diffuse the project efforts. For example, prior research has suggested that when members perceive a low likelihood of success on a project, they may increasingly take risks to improve their chances of success (Case \& Shane, 1998). If these activities and risks are outside of the existing project plan they may detract from official tasks and create additional chances for 
project failure. Finally, ongoing conflict may go unresolved throughout the project, inhibiting the ability to reach consensus and, consequently, preventing important actions from occurring at appropriate times.

The significance of this study is that it introduces a more complex understanding of communication as a framework for understanding project conflict and explores the ways that enduring conflict may be a precursor to project outcomes. That is, the current study explores how conflict in projects occurs as the existence of multiple versions of the "facts" that each makes sense to the various stakeholders. Because project resources are limited and individuals bring diverse needs and perspectives to their projects, such differences should be expected to result in conflict about important project decisions and activities. In this way, as conflict is constructed through communication and affects project decisions and activities, it may become a significant precursor to project outcomes. This contrasts with most existing research, where conflict is viewed as a characteristic of people to be overcome rather than multiple sources of meaning to be managed. For example, Frame (1995) suggests using the Myers-Briggs type indicator to diagnose the roots of conflict. From this approach conflict is understood to be a destructive force that emerges from a mismatch of personality traits. Project managers and their staffs are presumed to have (or even be selected because of) different traits and, thus, wise managers are instructed to surround themselves with staff that can "cover their weak points” (p. 73). However, such a belief that conflict is trait-driven and simply the consequence of a personality mismatch may serve to neutralize any motivation to understand the communicative nature of the conflict and, thus, forfeits the benefits that might be derived from its management. In addition, it fails to comprehend the positive outcomes that may emerge when conflict is framed as the source of important information to be managed.

In sum, while practitioners, consultants, and academicians have provided numerous behavioral heuristics and institutionalized "best practices” of project management into formal industry standards, an alarming number of high technology projects continue to fail. These failed 
projects appear to be managed under close scrutiny and under highly regulated processes. Yet, intractable problems still occur, and when discovered they often are recognized late in the project when few corrective actions remain viable. The current research effort addresses this problem by exploring how individuals create different systems of meaning for their project experiences and how those differences may come into conflict about important project topics. Through incongruent sensemaking these conflicts may become entrenched as the members begin to act in justification of their beliefs (Weick, 2001), potentially affecting the outcomes of their projects. In this way, enduring conflict is posited to reveal important underlying issues in high technology projects and possibly contribute a constitutive force in their outcomes. To this end, the current research intends to demonstrate how conflict can be recast as an opportunity to recognize and manage alternate meanings for important project issues, and to identify potential problems that may lead to project failure. 


\section{CHAPTER 2}

\section{Review of Literature}

This study explores how conflict operates as a precursor to failure in high-technology projects. The review of literature begins by describing the nature of the project management environment and then integrates the relevant literature on intraorganizational conflict. It then develops an interpretive framework to conceptualize conflict in a new way. That is, conflict is modeled as an incongruent sensemaking process where disparate meanings are constructed from the distinct perspectives of the participants that then become problematic during their involvement on projects. Finally, it provides the research questions to clarify the role of conflict in actual projects that may be indicative of their outcomes.

\section{Project Management}

The rapid trend toward globalization and technological advancement has ushered in to organizations a growing instability, driving them towards more open, complex, and political institutions (Heracleous, 2000; Thompson \& Richardson, 1996). This has resulted in greater uncertainty and more competitive pressures creating an incongruency with existing management structures that constrain information in hierarchical pathways and emphasize individual performance over group success (Ives, 2005). Consequently, organizations have searched for more effective management processes. To that end, project management has emerged and gained momentum in recent years as organizations have attempted to adapt to these new environments with greater cross-functional collaboration (Randolph \& Posner, 1992). As a first step in transitioning to a project-centric system, organizations have typically implemented a matrix structure of management (Thamhain \& Wilemon, 1975). Matrix management in organizations is characterized by dual lines of authority and responsibility where project and personnel management are separated and given different objectives (Goodman, 1967). Functional (line) managers have responsibility for a limited area of expertise and supervise employee specialists who perform specific skills. Conversely, project managers are accountable for project success 
and all of the component activities related to a specific project. Their objectives are typically to facilitate project completion by managing the three primary project metrics of time, cost, and quality (S. M. Davis \& Lawrence, 1977).

The matrix management approach has been successful because it is credited with a number of advantages in completing large projects. For example, it has allowed departments to maintain high levels of functional expertise while, simultaneously, permitting project managers to coordinate large numbers of diverse activities (Ford \& Randolph, 1992). Also, the matrix structure has been credited directly with facilitating the ability for experts to better solve complex technical problems (Knight, 1976). More generally, the matrix structure has been associated with improved project communication and coordination (Joyce, 1986; Larson \& Gobeli, 1987; Randolph \& Posner, 1992). This includes effective communication among the team members themselves, more contact across departments, quicker responses to problems, and better decisionmaking (S. M. Davis \& Lawrence, 1977; Denis, 1986; Joyce, 1986; Kolodny, 1979). Such improved communication helps organizations more efficiently adapt to changing conditions or take advantage of new opportunities (Jerkovsky, 1983; Stuckenbruck, 1982). Finally, matrix management has been attributed to greater employee satisfaction and improved motivation. For example, in a study of engineering managers, Denis (1986) concluded that matrix organizations result in greater teamwork, autonomy, participative decision making and greater individual development.

While the matrix concept is one component of project management, other elements have become adopted by many organizations through the creation of project management standards and commonly practiced in organizations. In the United States, the Project Management Institute (PMI) has become the de-facto standards organization representing the project management profession. It maintains a 'body of knowledge' that is officially distributed to members in the form of a guide known as the guide to the PMBOK (PMI, 2005a). According to this guide, projects are defined as 
A temporary endeavor undertaken to create a unique product or service. Temporary means that every project has a definite beginning and a definite end. Unique means that the product or service is different in some distinguishing way from all other products or services (PMI Standards Committee, 1996, p. 4).

The PMBOK identifies the project management function as existing within a hierarchal context and often driven by a strategic plan or portfolio of sub-projects arranged to achieve its sponsor's overall goals. Organizations that manage a number of ongoing projects may institute a project management office (PMO) to establish uniform practices and to coordinate the work performed by a number of project managers (PMI, 2005a).

According to the PMBOK standards, project managers are responsible for all aspects of a project including directing the resources of the project, its scope, quality, schedule and cost. The PMBOK guide assists this effort by defining 44 project management processes that are grouped in nine knowledge areas that represent the required tasks to effectively manage projects. The nine knowledge area categories are shown in Table 1.

Table 1: PMI - Project Management Areas of Knowledge

\begin{tabular}{lll}
\hline Integration Management & Scope Management & Time Management \\
Cost Management & Quality Management & Human Resource Mgt. \\
Communications Mgt. & Risk Management & Procurement Mgt. \\
\hline
\end{tabular}

Many of these processes such as scope planning, cost estimating, and risk identification are recognized as functions that are required in most projects. However, the PMBOK only provides a cursory framework for these processes by outlining basic terminology and approaches. Thus, the PMBOK (2005a) asserts that successful projects will also require the use of application specific tools and techniques to effectively perform each of the 44 defined processes. 
The PMBOK structure is limited to the activities associated with projects, but recognizes several overlapping functions that are involved in project success. To that end, the PMBOK (2005a) outlines three additional areas of expertise that it claims are required of project managers. These are: (1) knowledge of the project environment, (2) general management skills, and (3) interpersonal skills. Thus, the project manager is expected to possess a variety of organizational skills and orchestrate them successfully throughout the life of the project. This may involve different skills at different phases of the project based on its specific characteristics.

Projects are frequently composed of sequential phases that when taken together, make up the project life cycle (PMI, 2005a). Distinct phases within the life cycle are established to improve management control and cope with the uncertainty that exists throughout the development process (Cicmil, 1999). Typical phases might be: initiation, planning, execution, control, and closeout (PMI, 2005a). However, organizations adapt these basic phases and may include others to address their specific organizational requirements. For example, the pharmaceutical industry might include phases that apply to regulatory approval and clinical trials for drug development projects (PMI, 2005a). During a typical project, phase completions are marked by a specific deliverable that demonstrates the successful accomplishment of the phase requirements. Organizations frequently recognize these milestones through formal phase-release meetings and document each phase transition event. The final phase of the project represents deliverables which often define the successful completion of the project.

In spite of these highly formalized practices, Ives (2005), citing Peled (2000) has argued that:

Technical skills and tools associated with project processes will be unlikely to lead to success. Rather, success is determined by the project manager's skill in organizational politics, which are often the secret weapon for achieving the desired outcome. These political savvy project managers manage upward and outward and tailor their technological visions to the day to day reality of the organization (p. 40). 
This idea that important interpersonal processes and political considerations have more salience in project success than technical practices is not substantially addressed in the PMBOK nor reflected by its orientation. In addition, research has increasingly begun to challenge the dominant discourse of project management by questioning its widely accepted wisdom. For example, Williams (2005) has asserted that conventional project management concepts are based on three underlying assumptions that are supported by the PMBOK. First, is the assumption that traditional project management practices are rational and self-evidently correct. That is, the appropriate ways of managing projects follow generally accepted hierarchical control-oriented business models. This is said to result in a set of normative techniques that are automatically accepted and, therefore, do not require justification. Second, is an ontologically positivist orientation that posits a fixed truth applicable to all aspects of projects. Following this paradigm, the observer is independent of the observed and can be situated in a position that allows for an objective ability to examine and uncover the facts. The third assumption is that project management is fundamentally about managing scope, which can be deconstructed into smaller chunks with predetermined sequential dependencies. The idea of breaking down complex projects into simpler smaller tasks is seen by the PMBOK as an unproblematic method for resolving ambiguity. Williams (2005) argued that these three assumptions create a general orientation toward project management that results in the emphasis on very specific types of project behavior and leadership. These are (1) a heavy emphasis on planning, (2) a focus on the conventional modes of control, and (3) the view of projects as generally decoupled from the environment. Taken together, these establish a rationale for treating project management as a closed system of practices and procedures that can be scientifically examined for efficacy and, ultimately, optimized for success.

Others have concurred and suggested that the traditional approaches to project management represent an over-simplification of the project environment and a "lack of critical thinking by the practitioners who complacently accept seemingly reasonable answers, even if 
these answers lead to major failures” (Bredillet, 2004, p. 4). It is an over-simplification in that project management is modeled as a deterministic system with static operating parameters, a knowable scope, and unaffected by context. Winch (2004) argued that this represents a major limitation in project management theory.

Perhaps the major limitation of the systems analysis approach, which has been predominant theory in project management for the past 30 years, is that is it fundamentally deterministic. That is to say, it assumes that the scope of the project is completely knowable in advance, that an appropriate plan can be developed to deliver that scope, and that the problem of control is simply to keep the project delivery to plan (p. 42).

Essentially, he argues that actual projects are more complex than this and exist within a social structure that includes numerous dynamic and environmental variables. From this broader perspective, project management might be seen as having evolved into its own culture as its members have created a common identity defined and reified through their own ongoing organizing behaviors.

Project Management Culture

Beginning at the individual project level, participants are increasingly socialized into project management through formalized training and certification programs where they learn ‘appropriate’ project management behaviors. Further, as individuals participate in projects, their prior normative beliefs may have the consequence of creating a "presumption of logic" (Weick, 1987, p. 225), which encourages participants to assume that an underlying orderliness is at the root of their observations. According to Weick,

Most managerial situations contain gaps, discontinuities, loose ties among people and events, indeterminacies, and uncertainties. These are the gaps that managers have to bridge. It is the contention of this argument that managers first think their way across these gaps and then, having tied the elements together cognitively, actually tie them together when they act (p. 225). 
In this way, self-fulfilling prophesies are developed that explain prior beliefs, which over time become acculturated as basic assumptions about project management. Hatch (1993) described this as a shaping process of "proactive manifestation" (p. 662), where

prior assumptions provide expectations that influence perceptions, thoughts, and feelings about the world and the organization. These perceptions, thoughts and feelings are then experienced as reflecting the world and the organization (p. $662)$.

In her terminology, proactive manifestation is one of four interrelated processes that she described as constructing organizational culture and is built on Weick’s (1979) concept of enactment which is described elsewhere in the present paper. The growing standardization of project management practices has fueled this cultural element through the auspices of the PMBOK, the capability maturity model (McBride, Henderson-Sellers, \& Zowghi, 2004) and other standards which then lead to fundamental assumptions about how successful projects should operate. These standards then become self-perpetuating through the establishment of “communities of practice” (Garcia, 2005, p. 27) which adopt and promote them. Thus, a growing project management culture has emerged, largely atheoretically, and with its own assumptions about success.

Clearly a major purpose for adopting project management standards is the belief that 'best practices' can be applied across contexts to improve the lackluster results of project practices. According to Garcia (2005),

Why should we even try to normalize how different people approach an activity such as project management? Simply this: most software-intensive projects don't finish when they're expected to, they cost more than expected, and they don't do what the customer expected. By finding and codifying good project management practices, as a community we aim to increase future projects' success... Good standards can enable this process (p. 23).

However, Garcia clearly cautions that standards are often applied and are manipulated for organizational goals other than project effectiveness. Therefore, there is a need both to benefit from standard practices and, simultaneously, avoid the political and pragmatic risks associated 
with standardization. Overall, the abundant literature on project management has been functionally grounded and directed toward developing standardized practices and processes to guide projects toward success with an underlying assumption that 'success' is a relatively unproblematic construct. However, a growing body of research has questioned this assumption, which is a critical topic for the current study.

\section{Project Success}

While the PMBOK establishes clear procedures to define and document project goals, there has been a growing controversy about how goals are established and understood. For example, Pinto and Slevin (1987) have suggested that project success can be ambiguous because the project stakeholders have varying perspectives. Others have argued that the traditional view of projects being optimally successful by balancing the mix of time, quality, and cost is too simple to be realistic (Briner, Geddes, \& Hastings, 1990; Kleim \& Landin, 1992). For instance, some have argued that actual project success may have more to do with customer satisfaction and meeting users actual needs than in meeting specific contractual clauses (Avots, 1984). In other cases client satisfaction may be achieved while management objectives fail (Belassi \& Tukel, 1996). Similarly, Pinto and Mantel (1990) describe three aspects of project success that are distinct from the traditional cost, quality and time dimensions. These include (1) the implementation itself, (2) the perceived value of the project, and (3) client satisfaction. In this way, the ambiguity of actual project success may be related to factors that are not explicitly expressed in the formalized project plan.

Much of the prior research exploring success factors of complex projects has been developed from the underlying assumption that organizational practices and project manager characteristics are primarily responsible for project success (Belassi \& Tukel, 1996). In that regard, scholars have suggested any number of heuristics to guide practitioners toward successful projects. For example, Pinto and Slevin (1987) have published ten factors that underlie successful project completion (Table 2). 
Table 2. Underlying Project Success Factors

\begin{tabular}{|c|c|}
\hline Success Factor & Description \\
\hline 1. Project Mission & $\begin{array}{l}\text { Initial, clearly defined goals and general } \\
\text { directions }\end{array}$ \\
\hline 2. Top Management Support & $\begin{array}{l}\text { Willingness of top management to provide the } \\
\text { necessary resources and authority/power for } \\
\text { project success }\end{array}$ \\
\hline 3. Project Schedule / Plan & $\begin{array}{l}\text { A detailed specification of the individual action } \\
\text { steps for project implementation }\end{array}$ \\
\hline 4. Client Consultation & $\begin{array}{l}\text { Communications, consultation, and active } \\
\text { listening to all impacted parties }\end{array}$ \\
\hline 5. Personnel & $\begin{array}{l}\text { Recruitment, selection, and training of the } \\
\text { necessary personnel for the project team }\end{array}$ \\
\hline 6. Technical Tasks & $\begin{array}{l}\text { Availability of the required technology and } \\
\text { expertise to accomplish the specific technical } \\
\text { action steps }\end{array}$ \\
\hline 7. Client Acceptance & $\begin{array}{l}\text { The act of "selling" the final project to its } \\
\text { ultimate intended users }\end{array}$ \\
\hline 8. Monitoring and Feedback & $\begin{array}{l}\text { Timely provision of comprehensive control } \\
\text { information at each stage in the implementation } \\
\text { process }\end{array}$ \\
\hline 9. Communication & $\begin{array}{l}\text { The provision of an appropriate network and } \\
\text { necessary data to all key members in the project } \\
\text { implementation }\end{array}$ \\
\hline 10. Trouble-Shooting & $\begin{array}{l}\text { Ability to handle unexpected crises and } \\
\text { deviations from the plan }\end{array}$ \\
\hline
\end{tabular}

Others have assumed that all projects will have obstacles to success that can either be forestalled or overcome as the project unfolds. This belief fosters more emphasis on identifying and coping with risk and intervening in projects during problematic episodes to ensure their success. For example, as shown in Table 3, Cooke-Davies (2002) provides 12 factors that focus on risk management, monitoring, and intervention: 
Table 3. 12 Critical Success Factors

\begin{tabular}{|c|c|}
\hline Success Factor & Description \\
\hline 1. Education of Risk Management & $\begin{array}{l}\text { Adequacy of company-wide education on the } \\
\text { concepts of risk management }\end{array}$ \\
\hline 2. Organization Processes that Assign Risk & $\begin{array}{l}\text { Maturity of an organization's processes for } \\
\text { assign ownership of risks. }\end{array}$ \\
\hline 3. Proper Assignment of Risk & $\begin{array}{l}\text { Adequacy with which visible risks register is } \\
\text { maintained. }\end{array}$ \\
\hline 4. Risk Management Plan & $\begin{array}{l}\text { Adequacy of an up-to-date risk management } \\
\text { plan }\end{array}$ \\
\hline 5. Defined Organizational Responsibility & $\begin{array}{l}\text { Adequacy of documentation of organizational } \\
\text { responsibilities on the project. }\end{array}$ \\
\hline 6. Limit Project or Project Stage Duration & $\begin{array}{l}\text { Keep project (or project stage duration) as far } \\
\text { below } 3 \text { years as possible ( } 1 \text { year is better.) }\end{array}$ \\
\hline 7. Mature Scope Change Process & $\begin{array}{l}\text { Allow changes to scope only through a mature } \\
\text { scope change control process }\end{array}$ \\
\hline 8. Performance Measurement Integrity & $\begin{array}{l}\text { Maintain the integrity of the performance } \\
\text { measurement baseline. }\end{array}$ \\
\hline $\begin{array}{l}\text { 9. Effective Processes Between Project and } \\
\text { Functional Management }\end{array}$ & $\begin{array}{l}\text { The existence of an effective benefits delivery } \\
\text { and management process that involves the } \\
\text { mutual cooperation of project management and } \\
\text { line management functions. }\end{array}$ \\
\hline 10. Program / Project Practices & $\begin{array}{l}\text { Portfolio and program management practices } \\
\text { that allow the enterprise to resource fully a } \\
\text { suite of projects that are thoughtfully and } \\
\text { dynamically matched to the corporate strategy } \\
\text { and business objectives. }\end{array}$ \\
\hline 11. Program / Project Metrics & $\begin{array}{l}\text { A suite of project and program metrics that } \\
\text { provide direct feedback on current project } \\
\text { performance, and anticipated future successes, } \\
\text { so that project, program, and corporate } \\
\text { decisions can be aligned. }\end{array}$ \\
\hline 12. Lessons Learned Education & $\begin{array}{l}\text { An effective means of learning from } \\
\text { experience on projects that combines explicit } \\
\text { knowledge with tacit knowledge in a way that } \\
\text { encourages people to learn. }\end{array}$ \\
\hline
\end{tabular}


While numerous lists and heuristics have been developed from this perspective, the studies have been incomplete in their failure to more thoroughly account for the individual differences in project, team member characteristics, and the external environmental influences that occur (Belassi \& Tukel, 1996).

Taking a broader perspective, Belassi and Tukel (1996) have presented a framework of four factors related to project success. The researchers model success factors as interrelated characteristics and behaviors that exert a systemic influence on project outcomes. Their structure includes factors relating to the (1) project manager, (2) project, (3) organization, and (4) external environment. This work exemplifies an important shift in understanding project success by virtue of its focus on the interrelatedness of numerous causal agents. Some research has begun to appear that follows this broader and more systemic perspective. For example, Gray (2001) found five organizational characteristics that were positively associated with successful projects. These were: a type of climate or culture where individuals could engage in defining goals, questioning, free-expression, innovation, and individual satisfaction in the work. However, while such studies offer promise in their encompassing of individual and cultural variables, the static and contextually lean nature of their design fails to consider the significance of process and evolving meaning as issues to be negotiated. This may be particularly apparent in the context of decisionmaking meetings where project managers and their team members actually perform the prioritization or quantification of activities and make resource trade-offs for their project contingencies. The negotiation of interests during these meetings can be understood as an evolving process of decision-making exacerbated by high levels of complexity, uncertainty, and change. As a consequence, project complexity and decision-errors have been cited as mutually constituent factors that threaten project success (McCray, Purvis, \& McCray, 2002).

\section{Project Complexity and Decision Making}


Project managers are given the difficult and highly ambiguous task of managing a wide variety of resources and assets against the overarching constraints of scope, time, cost, quality, and stakeholder needs (PMI Standards Committee, 1996). Because high technology projects operate within an organizational context, these constraints are directly related to the larger organizational environment. Following Nguyen (2006), a number of traditional management issues impact the ability for project managers to make optimal project decisions. These include unmatched structures between the project team and the organization, a gap between processcentric and inter-process centric activities, and a mismatch of management scope to project resources. Collectively, these domains of misalignment are perceived to constrain information along with more tangible resources and limit the project team's ability to optimize their outcomes.

While a number of project management tools and practices are available, project success is still perceived to be predicated on the ability of project managers to make informed and valid decisions about these project tradeoffs (McCray et al., 2002). However, the voluminous workload and limited time encourages project managers to frequently rely on heuristics or rules-of-thumb to make most project decisions, which can foster a number of decision errors in projects. Because of this, McCray, Purvis, and McCray (2002) have noted that "project managers are surprisingly ineffective decision-makers” (p. 49). To that end, McCray, et al. have adapted Tversky and Kahneman’s (1974) typology to categorize the types of errors frequently occurring in complex projects. These are shown in Table 4. 
Table 4 - Decision Errors found among project managers

\begin{tabular}{|c|c|}
\hline Decision Error Type & Explanation \\
\hline Inappropriate comparisons & $\begin{array}{l}\text { Excluding important factors that were associated with } \\
\text { the prior objects of comparison. }\end{array}$ \\
\hline Misinterpretation of data & $\begin{array}{l}\text { Failing to identify and appreciate the variations } \\
\text { associated with previous data collection and its } \\
\text { outcomes. }\end{array}$ \\
\hline Reliance on profound events & $\begin{array}{l}\text { Profound events are more easily recalled and can } \\
\text { focus undo influence on their associated conditions. }\end{array}$ \\
\hline Misapplied risk & $\begin{array}{l}\text { Projects are erroneously viewed as more risky when a } \\
\text { large number of negative outcomes are identified. }\end{array}$ \\
\hline Anchoring & $\begin{array}{l}\text { An initial estimate becomes the 'anchor' } \\
\text { inappropriately affecting judgments about later } \\
\text { estimates. }\end{array}$ \\
\hline Preference for intuition & $\begin{array}{l}\text { Individuals tend to alter data based on their own prior } \\
\text { experience, even when the current situation is } \\
\text { dissimilar and there is no rational basis to do so. }\end{array}$ \\
\hline Bounded rationality & $\begin{array}{l}\text { Decision-makers have limited time and resources to } \\
\text { make decisions and, therefore, use a highly simplified } \\
\text { model of reality to make decisions about complex } \\
\text { events. }\end{array}$ \\
\hline Narrow beliefs or doctrine & $\begin{array}{l}\text { Beliefs often persist even after they have been } \\
\text { demonstrated to be false. Doctrinal beliefs are those } \\
\text { so valued, that they are closed to discussion. }\end{array}$ \\
\hline Failure to consider alternatives & $\begin{array}{l}\text { Scarce resources often are not allocated to } \\
\text { considering alternatives. Therefore, improved } \\
\text { solutions are forgone. }\end{array}$ \\
\hline Personal preference driving the approach & $\begin{array}{l}\text { Personal preferences are derived from past } \\
\text { experiences and individual factors that may prove } \\
\text { counter-productive in a current project. }\end{array}$ \\
\hline Succumbing to the gambler's fallacy & $\begin{array}{l}\text { The false idea that a positive outcome is 'overdue' if } \\
\text { a number of negative outcomes have preceded the } \\
\text { current situation. }\end{array}$ \\
\hline Tendency to be average & $\begin{array}{l}\text { Also, known as 'return-to-the-mean', this is failing to } \\
\text { accept that extreme performances are often rare, and } \\
\text { subsequent outcomes will be closer to average. }\end{array}$ \\
\hline
\end{tabular}


Escalating commitment

Preference for details

Overconfidence

Hindsight bias
Additional resources are often poured into a failing project as it continues to show greater signs of failure.

Individuals frequently are persuaded by evidence that is highly detailed, even if it is less advantageous than a solution that has less detail.

A misplaced belief that problems known now can be solved in the future, delaying their assessment and remedy.

The belief that a project who's outcome is known, could have been predicted all along.

Each of these 16 sources of decision errors is said to put projects at risk by negatively impacting time, cost, performance, quality, or functionality features. However, Purvis, McCray, and Roberts (2002) argue that such decision biases may be overcome by learning to recognizing them. In doing so, they argue that heuristics can be useful in providing simple approaches to problems but, simultaneously, the same heuristics can have both subtle and profound impacts. Such advice fails to provide convincing evidence that heuristics can be adequately managed to cope with project complexity. For the current study, the propensity for decision errors in projects is informative because it reveals the problematic nature of complexity in high technology projects and highlights the limitations of risk management as a solution.

The formal project management structure outlined by PMBOK is fundamentally deterministic (Winch, 2004) and follows the traditional organizational model of a cause-effect structure toward activities that is reminiscent of industrial-era technologies (L. E. Davis \& Taylor, 1976). In contrast, high technology projects require "people [to] operate in an environment whose ‘important events’ are randomly occurring and unpredictable” (p. 388). It follows that understanding how to interpret and respond to evolving technology is also confusing for the participants. According to Weick (2001),

new technologies are hard to diagnose because of the substantial mental demands they make on operators and the many ways in which surprises can 
occur; but new technologies are also hard to control because of interactive complexity, and they are hard to measure because people disagree about what constitutes effective performance (p. 153).

This is consistent with some recent research on IT projects, where project complexity affected group communication (Roberts, Cheney, Sweeney, \& Hightower, 2005). Less complex tasks involved more participation and interaction about both task and relational issues. Also, roles were better defined and more emphasis was placed on organizing. For moderately complex tasks, the researchers speculated that increased communication occurred because more members were comfortable with their roles and task knowledge, which led to greater participation. In this study, the concept of task complexity follows the definition by Wood (1986) where "complexity is a direct function of the number of distinct acts that need to be executed in the performance of the task and the number of distinct cues that must be processed in the performance of those acts” (p. 66). Wood's construct of task complexity portrays a direct relationship between the number of acts that must be performed and the knowledge and skill requirements that the team must collectively possess to be successful. Thus, project complexity would seem to construct the environment for conflict to become a central issue in project outcomes. This is essentially the argument of Ivory and Alderman (2005) when they describe complex organizational projects.

Projects are inherently internally fragmented, multi-nodal, and rife with numerous contradictory demands. Even at the simplest level, there are inevitably conflicting demands in projects; for example, between the demands of low cost, high quality, and quick delivery... whereby the simultaneous achievement of all three objectives is rarely possible without some compromise (p. 7).

They question the traditional wisdom about managing project complexity, which would suggest that complex projects can be broken down into smaller and smaller subprojects and tasks until they are manageable. The researchers argue that rather than eliminating complexity, this approach shifts the complexity to later stages that focus on integrating and coordinating a larger number of sub-tasks. The consequence of following such a strategy, is that the complexity of the 
project becomes hidden from management's attention until the later stages of project completion.

Further, these hidden issues may not appear until the customer has possession of the end product.

Recently, some research has suggested that project complexity should to be considered

within the context of environmental complexity creating four types of project management

models (Jaafari, 2003). This research suggests that project complexity interacts with

environmental complexity to create four models of project management. The four models are

described as (1) the ad hoc model, where project and environmental complexity are both low, (2)

the bureaucratic model where project complexity is low but environmental complexity is high,

(3) the normative model where project complexity is high but environmental complexity is low,

and (4) the creative-reflective model where project complexity and environmental complexity are

both high. In this research, the models represent different underlying approaches that guide

professional development, planning, and project practices. For the focus of the current study

where projects are inherently complex in and of themselves, the most salient models are the

normative model and the creative-reflective model. The normative model is described as the

rational approach and assumes that there is sufficient certainty and stability in the environment that it will be possible to define a set of goals and a framework for orderly planning and delivery of projects. Nearly all published bodies of knowledge, approaches by professional bodies, and their certification schemes underlie this model... Its limitation has already been reflected in reported project failures in complex IT and software systems, new complex products and organizational transformation (to name a few) (p. 55).

In sharp contrast is the creative-reflective model that recognizes how environmental complexity affects project outcomes.

This model relies on the principles of self-organization, and the insights and competence of the players in the project value chain. Practitioners in the model are empowered individuals, who possess a high degree of self-referential skills and are partial to change. Their functions transcend that of a rational processdriven approach to that of a creative approach through which they are able to achieve breakthrough solutions to optimally respond to both environmental and project complexity (p. 55). 
In regard to the current study, the significance of Jaafari's observations is in the discrepancy of how high technology projects are managed given differing considerations to the significance of environmental complexity. The difference in these two viewpoints is played out in the current study, which includes an exploration of how environmental complexity along with project complexity contributes to overall project conflict.

In sum, the project complexity that is associated with high technology projects serves to promote an environment where conflict emerges exclusive of the traditional view of conflict as a consequence of rationing limited resources. The more traditional view of conflict was articulated by Butler (1973) who argued that "conflict is inevitable in the complex social systems we call organizations because they have limited means with which to satisfy the divergent interests of their various publics” (p. 86). The distinction is important because the traditional view of conflict is derived from a functionalist perspective that represents a fixed-sum game where individuals vie for the same resources. In contrast, the present study adopts an interpretative approach, placing conflict in a broader role as representing incongruous meanings that project participants have for their project experiences as they unfold in changing environments. However, before expanding beyond this "limited resources” paradigm for conflict, it is important to review how conflict has been envisioned in organizations and specifically in the project management environment.

\section{Project Conflict}

Conflict has been seen as especially apparent in project management because of the matrix form described earlier. This form of management has been known to be more conflictladen than typical hierarchal management structures since it creates the environment where workers are taking instruction from two different authorities (Kirchof \& Adams, 1989). Some have even suggested that the matrix management system is built around introducing deliberate conflict between the functional and project managers (Cleland, 1968, 1974).

Three antecedent conditions have been claimed for conflict in projects. These are (1) ambiguous jurisdictions, (2) conflicts of interest, and (3) communication barriers and unresolved 
prior conflicts (Kirchof \& Adams, 1989). First, the ambiguous nature of projects has included uncertainty about technical issues (Katz \& Allen, 1985) and power struggles over areas of accountability and responsibility (Denis, 1986; Larson \& Gobeli, 1987). Much of this ambiguity emanates from team members who are working for two different individuals with diverse goals. Thus, even when each manager has clearly established objectives, ambiguity can exist among the team members who must know how to satisfy both superiors.

Second, conflicts of interest exist because project team members frequently have to make decisions based on tradeoffs from multiple stakeholders. Frequently, the project manager is at the center of conflicts over resources because he/she must negotiate with a number of functional managers / executives who all represent diverse organizational interests (Stuckenbruck, 1982). This can be further complicated in situations where a conflict of interest exists over the established processes and relationships among the members and managers (Katz \& Allen, 1985). For example, because the functional managers or executives retain responsibility for the everyday business of the organization based on standardized costs and budgeted resources, they are motivated toward maintaining stable processes. However, "the PM tries to overcome organizational inertia by changing existing patterns and relationships to serve the needs of his project” (Butler, 1973, p. 85).

Finally, communication barriers often exist among teams because they interact in an environment of prior relationships. Essentially, projects are conducted in bona-fide groups (Putnam \& Stohl, 1990) where existing relationships are affected by the members’ prior experiences with one another. Because prior conflicts may not be completely resolved, they may exert influence in existing projects (Kirchof \& Adams, 1989). The opportunities to resolve such conflicts may be mediated by the communication context of project teams.

The opportunities for effective communication to exist among team members have been debated. For example, Davis and Lawrence (1977) posited that the matrix structures should improve both the quantity and quality of messages communicated. However, some research has 
indicated that while the quantity of communicated messages increases, the quality may actually be decreased in a matrix project environment (Joyce, 1986). This combination of increased quantity and decreased quality follows Weick (1995) in that ambiguity occurs because there is too much information, not too little, and individuals must work to make sense of the information they receive. Also, because individuals participate and occupy distinct positions within their environment, they likely have access to different information and may come to adopt meanings that are role specific. In this way, it’s plausible that role conflict may emerge from differences among individuals based on their roles in a project or organization.

\section{Role conflict}

Role conflict has been widely discussed in organizational literature. It has been defined as consisting of three components (1) inconsistency of self and other expectations for role behavior, (2) disparate requirements of multiple roles, and (3) role overload (Rizzo, House, \& Lirtzman, 1970). In the project environment, this conflict is presumably greater because there are two authorities that control the role of the team member. In addition, the levels of authority are ambiguous in the project environment because a temporary role-reversal has been said to occur during projects (Butler, 1973). That is, functional managers may have to yield their power to the project manager during certain aspects of the project that their employees are engaged in. This may be exacerbated when departments operate with dual reward systems and dual accounting systems in an attempt to motivate their employees and track project costs (Kolodny, 1979). These uncertainties would suggest that role conflict would be more significant in the project management structure. However, Joyce (1986) found no differences between role conflict in traditional versus project structured management. This may result from the propensity of organizations to carefully prescribe roles for individuals in projects, in which they spell out minute details of their responsibilities (Knight, 1976). However, Goodman (1967) questioned the degree to which organizations may work to clarify roles and even suggested that roles may be left 
deliberately ambiguous. Thus, additional research needs to clarify the issue of how role conflict occurs in matrix organizations.

In sum, organizations have widely adopted a project management strategy that is based on a matrix-management platform and created an atmosphere where team members are highly interdependent, yet limited by project complexity and ambiguous criteria for success. This has resulted in experts offering heuristic advice implying that specific prescriptive behaviors can avoid most project problems and the corresponding project conflict. This approach has several underlying assumptions about the general nature of conflict that is addressed by the more theoretical and comprehensive discussion that follows.

\section{Conflict Research}

Conflict research can be divided into three conceptual arenas (Nicotera, Rodriguez, Hall, \& Jackson II, 1995). First, macro-level research covers institutional, geo-political, and social interests. It often includes longstanding historical claims along with ongoing cultural, religious, and political disputes. From this perspective, conflict operates between groups or entire nations. At this level, there has been an extant literature about the philosophy of conflict and an ongoing debate about its theoretical underpinnings. Fink (1968) provides an exhaustive discussion on this topic.

Second, the game-theoretic research is the analysis of rationally conducted conflict between "players” (participants or opponents), each of whom pursues well-defined interests and chooses among alternative courses of action. (Steinfatt \& Miller, 1974). It provides a mathematical and logical basis for conflict and develops solution sets based on sequences of moves and counter-moves. This view assumes that the players are consistently rational and will continually make decisions in their own best interest. The Prisoner's Dilemma is an example of a logical conflict game that requires individuals to make choices between competing and cooperating in a conflict situation (Steinfatt \& Miller, 1974). However, a major limitation of this approach has been its failure to examine the role of communication and social behavior as a 
factor in conflict (Bonacich, 1970; Shubik, 1970). For example, game theory generally does not consider limited rationality, the role of emotions in decisions, or altruistic cooperation.

The third research area, which is relevant to the current study, is the micro-level behaviors that form a cognitive and communication basis for conflict behaviors. The preponderance of research on interpersonal and organizational conflict originates from the microlevel perspective. At this level, researchers have varied considerably in what conflict is.

According to Pondy (1966)

Conflict is a concept with many meanings. It is quite legitimate to think of conflict as interpersonal hostility; but it is equally legitimate to think of it as a disagreement or perception of disagreement between two persons on some choice or preference; or as the inability to resolve such disagreements; or merely as incompatibilities among several formally defined jobs. (p. 246)

This variety of thinking about conflict has led to a broad and diffuse research agenda where scholars have approached conflict from a number of different directions. Much of this research has evolved from a functional positivistic perspective, which proceeds from an underlying assumption that conflict is primarily negative and destructive (Nicotera, 1995).

Functional Perspective

Researchers who have investigated conflict from the functional perspective have generally viewed conflict as the consequence of either a personality trait or as a consistent style of behavior that emerges from contesting limited resources. Such a fixed-pie approach predisposes at least one party to fail and, thus, creates the tension and strife among the participants that many people equate with conflict. Many of the definitions for conflict proceed from this basis and some typical examples are listed in Table 5. 
Table 5. Conflict Definitions

$\begin{array}{lc}\text { Conflict Definition } & \text { Source }\end{array}$
Any social situation or process in which two or more social entities
are linked by at least one form of antagonistic interaction.

A struggle over values and claims to scarce status, power, and resources in which the aims of the opponents are to neutralize, (L. A. Coser, 1967, p. 8) injure, or eliminate the rivals.

Conflict exists whenever incompatible activities occur . . . an action which, prevents, obstructs, interferes with, injures, or in some way makes it less likely or less effective.

A situation in which interdependent people express (manifest or latent) differences in satisfying their individual needs and interests, and they experience interference from each other in accomplishing these goals.

Conflict arises when a difference between two (or more) people necessitates change in at least one person in order for their engagement to continue and develop. The differences cannot coexist without some adjustment.

Conflict is an expressed struggle between at least two interdependent parties who perceive incompatible goals, scarce

(Deutsch, 1973, p. 156) resources, and interference from others in achieving their goals.

Definitions such as these privilege a functional understanding of conflict for a number of reasons. For instance, these definitions tend to be reductionistic in nature focusing on one or two essential ingredients, thus, excluding the complexity of situational variables and recognizing how context alters the essential meaning of an event (Rubin, 1978). They are also static, in failing to acknowledge the dynamic and unfolding nature of reality (Hawes \& Smith, 1973). Collectively, such simplifying assumptions predispose researchers to fix experimental conditions and search for stable differences in individual responses. This focus has led conflict researchers to identify and emphasize individual traits as the salient constituents of conflict. 
Conflict as Individual Traits. While scholars have frequently studied communication variables as the consequence of personality traits (Levine \& Boster, 1996). Such research has frequently failed to explain substantial amounts of variance in human behavior (L. Ross \& Nisbett, 1991). Consequently, its proponents have emphasized its value in developing theoretical constructs. From this viewpoint, a significant body of conflict research has investigated argumentativeness (Infante \& Rancer, 1982) and verbal aggressiveness (Infante \& Wigley, 1986) as personality traits leading to conflict.

Argumentativeness has long been seen as a communication trait characteristic which influences message selection and use (Boster \& Levine, 1988; Boster, Levine, \& Kazoleas, 1993; Infante, 1981). Infante and Rancer (1982) defined argumentativeness as “ a generally stable trait which predisposes the individual in communication situations to advocate positions on controversial issue and to attack verbally the positions which other people take on these issues” (p. 72). When compared to "low argumentatives," individuals who are labeled as "high argumentatives” are perceived to argue more, be more interested in the issues of the argument and less flexible to change their minds. Other findings suggest that observers will perceive "high argumentatives” as higher in expertise (Infante, 1981) and more credible and competent (Onyekwere, Rubin, \& Infante, 1991).

Verbal aggressiveness has also been considered a personality trait and distinct from the concept of argumentativeness (Infante \& Rancer, 1982). Verbal aggressiveness is "a personality trait that predisposes persons to attack the self-concepts of other people instead of, or in addition to, their positions on topics of communication” (Infante \& Wigley, 1986, p. 61). This distinction describes whether individuals will exhibit a predisposition toward attacking the positions that others hold (argumentative) or make personal attacks on their character (aggressive).

Presumably, these trait characteristics provide utility by offering individuals a method to understand, describe, and predict the behaviors of others. However, some have argued that individuals may simply take on the identity of a specific trait, and then they will act in its service. 
For example, Hocker and Wilmot (1995) describe how individuals develop certain conflict predispositions: "People who perceive themselves as powerless usually do not talk effectively about their own needs and, after a while, may adopt a self-defeating, accommodating style that becomes fixed” (p. 90). This is similar to Cooley’s (1964) concept of the looking-glass self where perceived social reactions are sufficient for individuals to preemptively adopt specific communication patterns. Thus, a shortcoming of using the personality approach as a predictive tool is that it generally fails to consider the social and emergent nature of conflict.

Since dyadic communication situates two individuals in the conflict exchange, some scholars who have suggested a personality basis for communication assert that the personality of both parties contribute to the interaction (Thorne, 1987). In this way, considering a transactional approach toward communication "offers a dynamic and more realistic alternative approach to the study of personality” (p.718). For example, using the personality construct of introversionextroversion, Thorne found that matched extrovert dyads spent significantly more time on pleasure than problem talk, when compared with mixed dyads. By demonstrating that extroverts actually change their communication patterns based on the partner's characteristics, such a finding challenges a predominately trait-based approach to conflict. This is consistent with other studies on conflict where the personality of both participants has been posited to contribute to the outcomes of conflict situations (Herrmann \& Kogan, 1977; Oltman, Goodenough, Witkin, Freedman, \& Friedman, 1975; Slack \& Cook, 1973). The cumulative weight of such findings tends to deemphasize internal processes and implicates communication and behavior as important components in conflict. Following this path, social scientists have searched for consistent patterns of conflict behavior that people select independently of or in concert with specific others. This has led to the concept of conflict styles as a two dimensional construct where both an individual's concerns and the other participant concerns are accounted for.

Conflict Styles. Peoples’ conflict behaviors have been typed and classified in many different ways resulting in the classification of frequent behaviors into specific styles (Putnam, 
1988). Conflict styles have been defined as "patterned responses or clusters of behavior that people use in conflict” (Hocker \& Wilmot, 1995, p. 96). However, the use of this term varies among researchers. Some reserve style for trait-like dispositions (Conrad, 1991) while others use style as a behavioral construct (Wall Jr., Galanes, \& Love, 1987). Folger, Poole, and Stutman (1993) reported that conflict styles could be viewed from three perspectives (1) as a property of the person, much like personality, (2) categories of behavior, and (3) communication orientations toward conflict.

Most current conceptions of conflict style have evolved from Blake and Mouton’s (1964) Managerial Grid (Figure 1), which was established to represent the conflict that managers experience with their subordinates.

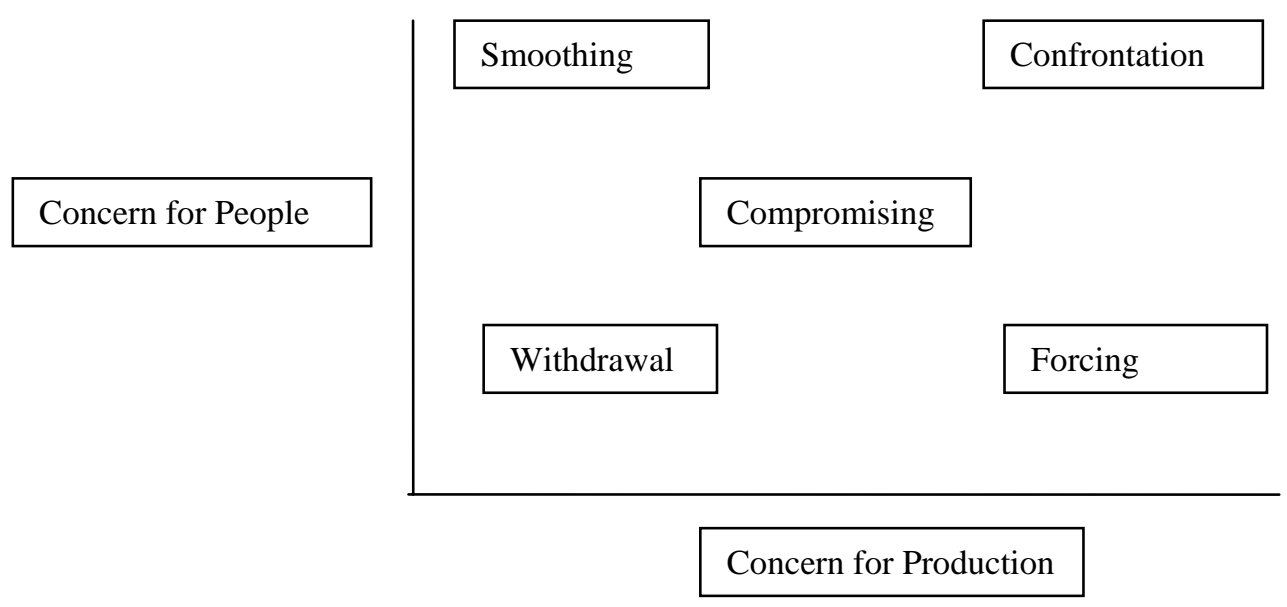

Figure 1: Blake and Mouton Grid

In this grid, managers are typed based on differences they exhibit in two orthogonal dimensions, concern for people and concern for production. In this model forcing corresponds to a high concern for production and a low concern for people. Confrontation is the label for managers scoring high on both dimensions. Withdrawal represents a low score on both dimensions. 
Smoothing is a low concern for production and a high concern for people. Finally, compromise is the middle area on the two dimensional chart. According to Blake and Mouton, managers will develop a consistent preferred style which represents their dominant approach toward interacting with subordinates. However, they may resort to a backup style if the dominant style proves to be ineffective in a particular case.

This two-dimensional approach toward conflict became the framework for Thomas and Kilmann’s (1974) conflict modes. This modification of the Blake and Mouton grid provided a generalized explanation for the existence of conflict between individuals. In the ThomasKilmann configuration, one axis represents a concern for self while the second axis represents concern for another. Over time, this became a historically important theoretical framework for conflict by its positing of a two-dimensional structure for the existence of interpersonal conflict (Ruble \& Thomas, 1976).

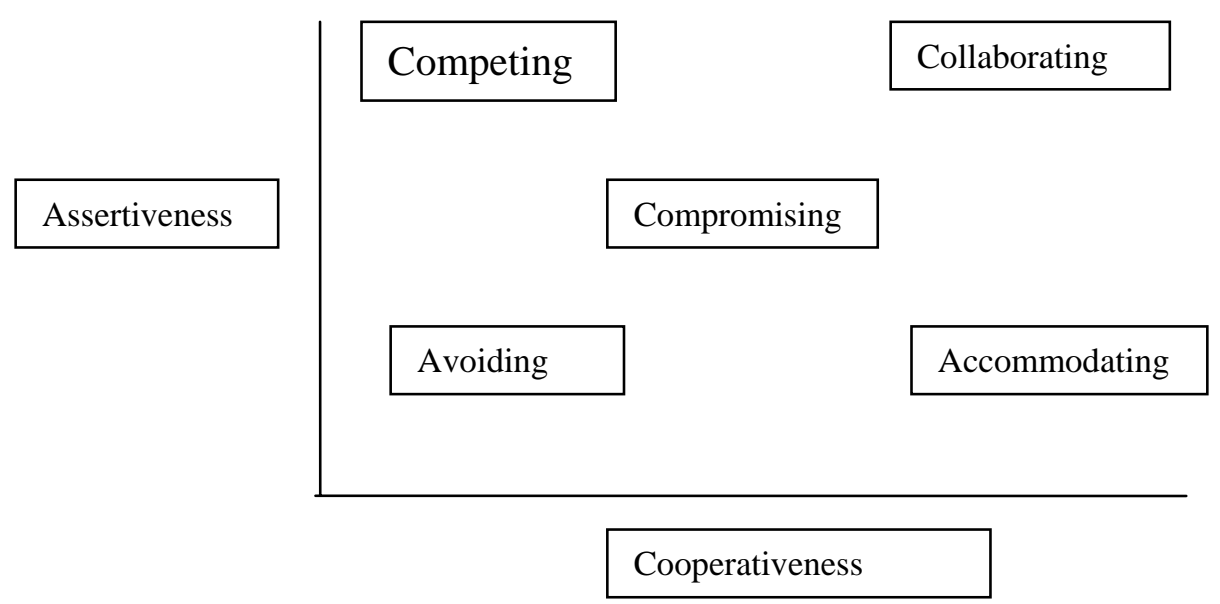

Figure 2 - Thomas - Kilmann Conflict Styles

The Thomas-Kilmann (1974) instrument provides a means for placing individuals into one of five categories based on two underlying dimensions. The dimension of assertiveness represents one's 
concern for self while the dimension of cooperativeness represents one's concern for the other. There are five distinct types of individuals represented by the two dimensions (Thomas \& Kilmann, 1974, p 8). Being low on both dimensions represents the avoiding type. Avoiders are perceived to be unassertive and uncooperative often delaying an issue for a future time. They are also characterized by the propensity to withdraw from an uncomfortable or threatening situation.

Individuals who score high on the assertive dimension and low on the cooperative dimension are labeled as competing. These individuals pursue their own interests at the expense of the other. It has been identified as a power-oriented mode where individuals use whatever resources the deem appropriate to meet their own needs (Thomas \& Kilmann, 1974, p. 8).

Individuals who are low on assertiveness but high on cooperativeness are described as accommodating. Accommodators are those who neglect their own concerns in a self-sacrificing way to meet the needs of others. This may take the form of generosity, charity, or obedience to yield to the other point of view (Thomas \& Kilmann, 1974, p. 8).

Individuals who score high on both assertiveness and cooperativeness are described as collaborators. These individuals attempt to find solutions to conflict that meet the needs of both parties. This involves "digging into an issue to identify the underlying concerns of the two individuals and to find an alternative that meets both sets of concerns” (Thomas \& Kilmann, 1974, p. 8).

The middle area represents compromising behaviors. Compromising is an attempt to meet some of the needs of each party, although neither party will be fully satisfied. Its advantage is to find and expedient solution, although doing so may overlook a fully satisfactory solution . (Thomas \& Kilmann, 1974, p. 8).

It is important to note that the Thomas-Kilmann conflict modes are theoretically distinct from the Blake and Mouton grid concept. Blake and Mouton envisioned their grid of conflict styles to be quite stable based on power differences, personal values, and personality factors 
(Womack, 1988a). In contrast, the Thomas-Kilmann Mode styles included both personality and situational factors such that individuals could shift more easily to alternate styles.

The elegant conceptual appeal of the Thomas-Kilmann grid found its roots in prior work and generated numerous variations. For instance, Walton and McKersie’s (1965) framework provides integrative and distributive dimensions. The distributive dimension represents choices between competing and cooperating while the integrative dimension ranges from collaborating to avoiding. Hall’s (1969; 1986) Conflict Management Survey (CMS) described the two dimensions as concern for personal goals and concern for relationships. These dimensions result in the styles: win-lose, yield-lose, lose-leave, compromise, and synergistic. Hall's style instrument was based on the concept that all five styles are equally available to all participants and that everyone uses all of the styles at different times. However, because people are perceived to have patterned behavioral predispositions, individuals are expected to use some styles more frequently than others (Schockley-Zalabak, 1988).

Other versions have included Pruitt and Rubin's (1986) four style configuration that leaves out compromise, Rahim’s (1983) five style conflict inventory that includes assessing the amounts of conflict at different levels, and Canary and Spitzberg’s (1987) three conflict styles relating to communication competence. Canary and Spitzberg indicate that individuals adopt integrative, distributive, or avoidant styles, which are based on effectiveness and appropriateness in specific communication conflict interactions. The criticism that these configurations leave out an important emotional component was remedied by Nicotera who introduced emotional intent as a third dimension to the conflict space (Nicotera, 1993). According to Nicotera, the two dimensional structure serves to limit theoretical understandings and may not provide the basic mechanisms that drive behavior. For example, individuals may adopt a specific interaction style such as avoiding, not only because it represents a low concern for the other, but also because the particular situation is emotionally uncomfortable for the individual. In this way, affect becomes 
an important factor that is perceived to mediate the expression of conflict styles in actual conflict behavior.

This assumption that relatively stable conflict styles or traits exist and become important factors in conflict behaviors has fostered considerable research toward their measurement. Typical measures involve self-report survey instruments that ask participants to code their own behaviors. However, many of these have been criticized for a number of weaknesses. In response, Putnam and Wilson (1982) developed the Organizational Communication Conflict Instrument (OCCI) to improve both conceptual and methodological weaknesses of the prior conflict instruments (Wilson \& Waltman, 1988). Specifically, they noted the importance of administering conflict instruments within the context of a specific conflict situation. Through factor analysis, and using hypothetical situations, the researchers found three styles that they identified as categories of conflict strategies. The resulting strategies are non-confrontation strategies, solution-oriented strategies, and control strategies. While the OCCI data results suggest that individuals within organizations use solution-oriented strategies most frequently, Wilson and Waltman (1988) indicate that social desirability bias may skew the results and that in actual conflict situations, anti-social strategies may dominate when identities or important tasks are threatened. However, OCCI is significant in recognizing the influence of situational factors in conflict behavior.

Another variation is the Ross-DeWine (1982) conflict management message style (CMMS) that utilizes verbal messages as items. The CMMS identifies three types of messages: self-oriented, issue-oriented, and other-oriented (R. G. Ross \& DeWine, 1988). This instrument requires the subject to participate in a critical incident recall by writing a description of their “most common behavior in conflict” (p. 392). Through a comparison process with various types of conflict messages, individuals are rated on three conflict sub-scales. While unique in its methodology, this instrument deemphasizes context to assign a specific style. Interestingly, the authors report that trainees using this instrument often complain about its content sterility, 
indicating that how they would respond to an actual conflict situation 'depends on where I am, and with whom I'm talking' (p. 407).

In sum, a number of researchers have developed conflict style instruments in association with their theoretical approaches toward conflict, which are shown in Table 6.

Table 6 - Conflict Style Instruments

\begin{tabular}{ll}
\hline Instrument & Source \\
\hline Conflict Management Survey & (Hall, 1969) \\
MODE Survey & (Thomas \& Kilmann, 1977) \\
Organizational Conflict Inventory - II & (Rahim, 1983) \\
$\begin{array}{l}\text { Organizational Communication Conflict } \\
\text { Instrument }\end{array}$ & (Putnam \& Wilson, 1982) \\
$\begin{array}{l}\text { Conflict Management Message Style } \\
\text { Instrument }\end{array}$ & (R. Ross \& DeWine, 1982) \\
\hline
\end{tabular}

Womack (1988b) reviewed each of these and provided instrument reliability comparisons and a summary of strengths and weaknesses. She concludes that all the instruments suffer from a theoretical weakness that confounds situational and personality factors as the underlying component of measures.

Envisioning conflict as a style of behavior has also had other criticisms. Even from a functional perspective, measuring individuals' conflict styles has been problematic. For example, Hocker and Wilmot (1995) articulate several limitations for those who would use the conflict style instruments. These include (1) self-reporting biases rather than actual behaviors, (2) lack of process orientation, (3) a false impression of situational stability, and that (4) measuring styles ignore the relationship dimension of an actual conflict (exception of Nicotera, 1993). Consequently, such issues call into question whether the style concept is consistent enough to be widely effective. 
The consistency of conflict style behaviors has been investigated by a number of researchers (Sternberg \& Dobson, 1987; Sternberg \& Soriano, 1984). For example, in a study of hypothetical conflict situations, Sternberg and Soriano studied 32 college students to ascertain their preferred resolution styles given seven possible alternatives (physical action, economic action, wait and see, accept the situation, step down, third party interventions, and undermine self-esteem). The researchers indicated that their results demonstrated consistency of participant preferences across a number of conflict scenarios. However, while Pearson product correlations ranged from 0 to .64 across the seven response alternatives for the organizational context and only four of the seven were significant. Nevertheless, the authors concluded that these represented "more or less preferred styles of conflict resolution” (p. 215). Overall, the relatively weak evidence supporting the style concept appears to contradict its popular usage but has been frequently subsumed by its normative appeal. Still other problems related to the use of conflict styles are more conceptual in nature.

Some conceptual difficulties with the style concepts focus on whether the styles actually are outcomes of the underlying dimensions. For example, the Thomas-Kilmann MODE instrument has been questioned on this basis (Womack, 1988a). Contrary to the MODE designation that avoidance should correspond to a low concern for self and others, it is plausible that individuals avoid conflict in other cases as well. For instance, some individuals may avoid conflict simply because they have a very high concern for others and perceive the resulting interaction would be relationally damaging. Because of this, some scholars have ironically suggested that the notion of conflict styles does not reflect "the way it really is" and, therefore, use the construct only as a teaching device (Hocker \& Wilmot, 1995, p. 97). Another issue is that conflict styles have been defined from a decidedly Western perspective. In other words, the conceptualization of self and other has been highly differentiated in Western cultures (Gibson, 1997) and fails to appreciate the values associated with a collectivistic culture. Some research which is attempting to recover the principle of conflict styles from this broader intercultural 
perspective challenges the basic construction of the self-other distinction (Brew \& Cairns, 2004; Leung, Koch, \& Lu, 2002).

Since the publication of the original Thomas-Kilmann instrument, Thomas (1992) has refined the concept in terms of the meaning given to the conflict-handling modes. In a subtle change, Thomas suggests that the modes should be thought of as "strategic intentions" (p. 269) rather than behaviors or orientations. Another refinement is his emphasis on the two-dimensional model being purely a classification scheme or taxonomy. The significance of these changes is to distance the model from claims that it can explain or predict actual behaviors. Rather, these represent motivations that are combined with other factors to express behaviors. Thomas (1988) expressed concern that the causal models such as Blake and Mouton (1964), Hall (1969), Rahim and Bonoma (1979), and Pruitt (1983) all emphasize one’s valence (i.e. level of concern) for possible conflict outcomes. As causal determinants, Thomas suggests that the two dimensional conceptualizations are overly simplistic and, therefore, of limited use. Consequently, Thomas models conflict outcomes in terms of both cognitions and intentions, along with contextually dependent judgments and emotions. Thus, according to Thomas (1988) “during the episode [of conflict], these cognitions can also take different courses as a result of the other's behavior, so that one's conflict modes can change or evolve during interaction” (p. 434). This is supported by Baxter (1980) who found that individuals use different styles during different phases of small group decision making. Therefore, even when viewing conflict from a style framework there is likely to be considerable variation in how individuals handle conflict across different situations. Because of this, Thomas (1988) has since preferred to avoid the term style, in lieu of mode. In addition, he suggests that "it seems more accurate to discuss an individual's 'profile' or 'mix' of modes” than a singular mode (p. 435). Thomas argues that this conclusion is supported by the construction of the conflict style measures:

The instructions on instruments that measure the modes tend to ask for these mixes-asking individuals for aggregated judgments of their modes across different episodes and relationships. It is important to realize that these mixes 
(and thus the scores on these instruments) do not solely reflect individuals' predispositions, but also differences in the kinds of situations faced by the individuals (Thomas, 1988, p. 435).

In sum, the extant literature on conflict styles is misleading to the extent that it appears to provide a causal explanation for conflict behaviors. Also, it is especially misleading in equating conflict styles with behavioral predispositions or personality traits that operate independently of relational or contextual factors. This is supported by Knapp, Putnam, and Davis (1988) who argue that researchers need to establish methods that uncover how conflict behaviors are shaped by evolving interactions that are contextually dependent and relationally constrained. To that end, some have argued that the trajectory of conflict in actual relationships is primarily directed by the interpersonal competence of the participants.

Conflict as Interpersonal Competence. Competence based approaches to organizational conflict have examined interpersonal and individual interaction processes (Papa \& Canary, 1995), which has led to a number of observations and theories about specific communication characteristics that are indicative of conflict (Spitzberg, Canary, \& Cupach, 1994). First, conflict emerges when individuals form impressions about messages in a specific context (Spitzberg, 1983). Second, competence based approaches have primarily been known for relating the factors of effectiveness and appropriateness to the conflict situation (Canary \& Spitzberg, 1987, 1989; Spitzberg \& Canary, 1985). Both variables are framed within a relational context where the dyadic participants jointly agree on what messages are both appropriate and effective.

According to Papa and Canary (1995) a competence based approach to conflict differs from the style concept in at least two ways. First, competence implies an evaluation of intrapersonal behavior in contrast to styles, which is simply descriptive. This suggests that the evaluation process is useful to gain insights that provide behavioral choices during the conflict situation. Second, the competence construct incorporates the significance of context such that different conflict behaviors are appropriate for different situations. However, both the competence and the style approach tend to be static models, which suggest that conflict behaviors 
can be predicted in advance. Also, these two models of conflict appear to be somewhat related in that some studies have indicated that certain conflict styles appear to be more competent than others in most situations. For example, cooperative and collaborative styles have been found to significantly correlate with both appropriateness and effectiveness (Canary \& Spitzberg, 1987, 1989, 1990). This may occur because there are norms or scripts for behavior that occur within specific cultural environments, which will be discussed in detail in the next section.

Appropriateness is defined as consisting of behaviors that do not violate relational or situational rules while effectiveness is generally seen as meeting relational objectives. However, since relational goals are often unspoken (Wilmot, 1987), competence is highly subjective and represented by private meaning systems of the participants. This creates both a conceptual and a methodological problem for envisioning conflict competence as a static message-centric skill. From a methodological perspective, the communication competence construct has been problematic because it has often been measured from a priori assumptions even though it is considered a contextually dependent variable (Wellmon, 1988). From a conceptual standpoint, conflict competence instruments are problematic because they fail to measure the negotiation of meaning that evolves during an unfolding conversation. Evidence for this includes Lakey and Canary’s (2002) study which linked competence assessments with an actor’s own goal achievement as well as his / her sensitivity to a partner's goals. Actors who were more sensitive to their partners’ goals and achieved their own goals assessed conflict as more competent. Partner sensitivity alone proved to be a strong predictor while the actor's own goal achievement was a moderate independent predictor. These findings provide a challenge to a purely intrapersonal reading of the competence model because they take on a context dependent form that is no longer limited to the intrapersonal performance evaluation described by Papa and Canary (1995).

This criticism is somewhat abated for the updated competence model where "episodic factors” (p. 79) operate to mediate the conflict relationship link (Canary, Cupach, \& Serpe, 2001). 
This newer model posits that both contextual and relational factors should be included in interpreting the meaning of an episodic exchange. In effect, "because interpretations of behavior are qualified by and derived from the context, the same behavior can take on different meanings in different episodes” (p. 81). This model also attempts to incorporate partner responses as part of the competence construct. In addition to appropriateness and effectiveness the researchers include “one’s [partner’s] felt satisfaction with the interaction” (Canary et al., 2001, p. 82) as a factor in conflict communication competence. This latter factor seems curiously discordant with the concept of competence unless partner satisfaction can be reduced to determinant variables relating one person's behavior to another person's feelings. Also, felt satisfaction may only occur after the fact and could be related to the outcome of the conflict rather than the process itself. However, it seems reasonable in a global statistical sense for some behaviors to be more satisfying than others in most situations, which would provide support for this model. Another limitation of this model is that it does not consider the evolving interaction processes that occur during a single episode.

In sum, the competence perspective serves to introduce the significance of situational effects as variables influencing the conflict behaviors of the participants. However, this approach is static and still represents an oversimplification of individual behaviors for conflict in situ, primarily because it fails to address the evolving processes of negotiated meanings as individuals participate in shared experiences. This deficit is also demonstrated by considering the role of culture in conflict behavior, which is ignored by the traditional focus on behavioral predispositions.

Conflict and culture. The lens of culture provides an opportunity for researchers to incorporate less tangible criteria in understanding the nature of conflict. While cultural insights often lend themselves to a constructionist ontology, much of the cultural investigation into organizational behavior has nevertheless followed the functional path. This work has ranged 
from investigating micro-cultures formed at the small group level to the influence of nations and ethnic groups on communication.

The impact of national culture on conflict has provided theoretical perspectives such as Ting-Toomey’s (1988) face-negotiation theory of conflict. Her theory argues that conflict represents a potentially threatening situation and individuals are motivated to protect their identities in such circumstances by engaging in specific communication exchanges referred to as facework (Goffman, 1959). According to Ting-Toomey, national cultures produce individuals who vary in their need for face and, therefore, culture becomes predictive of disparate conflict handling styles. This is consistent with Hofestede (1980), who suggested that individualist and collectivist cultures foster different values for the significance of self in a conflict with others and, consequently, differences my be expected in preferred conflict styles. For example, in a study of Anglo-Australians and Chinese university students, Brew and Cairns (2004) found confirming differences between groups in the value of assertive conflict styles, with the more individualistic group (Anglos) rating assertive conflict as more valued. The dominant styles adopted by the Anglos were results-oriented, (labeled as a direct style) and solution-oriented (labeled as a diplomatic style). In contrast, for the Chinese, the styles which emerged were passive (labeled as an indirect style) and solution-oriented (direct). A third prediction based on Ting-Toomey and Kurogi’s (1998) theory that power distance (another Hofestede variable) would be implicated in conflict style behavior was not supported. However, the authors speculated that this could have been a methodological artifact. Others have also found mixed support for the relationship between culture and conflict style. In one study, conflict style preferences were measured among students representing 31 different countries (Cai \& Fink, 2002). According to Cai and Fink, among the five conflict styles that were measured (avoiding, obliging, integrating, compromising, dominating) the avoiding style was preferred more by "individualists” while the compromising and integrating style were preferred more by “collectivists.” However, there were no significant differences for the obliging style. Most notably, Cai and Fink found that (except for the 
dominating style) the meaning of each style of conflict was interpreted differently between collectivists and individualists. Other researchers concur with this methodological difficulty and argue that the individualism-collectivism construct actually conflates a number of underlying attitude and valued driven constructs (Leung, 1988; M. W. Morris et al., 1998). These studies illustrate how individuals bring their national and ethnic backgrounds into organizations and how those cultural characteristics appear to alter both the meaning and the course of conflict.

While culture has often been viewed at national and ethnic levels, researchers have also suggested that cultures exist in organizational and sub-organizational units (Hofestede, 1997; Schein, 1992). At the organizational level, culture may be thought of as "a historically grounded system of shared assumptions, ideas, beliefs, and related patterns of behaviors learned by a group of people over time, resulting from their collective experiences” (Baba, Falkenburg, \& Hill, 1996, p. 44). Similarly, Deal and Kennedy (1982) defined corporate culture in terms of the values, heroes, rites, rituals, and communication networks that exist in organizations. However, numerous scholars have focused on the development and effects of cultural segments within organizations. For example, in describing organizational sub-cultures, Schein (1992) detailed how management and IT subcultures can collide by possessing incongruous assumptions that guide their actions. For Schein, underlying assumptions represent the most basic level of culture, which he defined as "unconscious, taken for granted beliefs, perceptions, thoughts, and feelings" (p. 17). Because this level of culture is not directly accessible, it is only indirectly observed through espoused values and, in turn, observable artifacts. These three levels create enduring differences between groups that are sometimes difficult to identify and are resistant to change. Hansen and Kahnweiler (1997) have documented how organizational executives represent a distinct group with shared perspectives that transcend organizational boundaries. In this way, certain members within organizations who have common roles and perspectives are posited to construct occupational cultures through a process of "subcultural patterning” (p. 118). This occurs when individuals believe they have the exclusive right to perform a given set of 
interrelated tasks. Through their collective experiences these individuals establish routines and norms that exist apart from those who occupy distant positions.

Cultural differences are important in conflict because they create schematic perceptions that filter user experience (Gertz, 1973; Rice, 1980). Such activities are constitutive of a culture to the extent that they combine and reinforce a cohesive meaning, in contrast to others who bring different backgrounds and diverse experiences to their positions. Within each group these cultural values may serve to produce a rhetorical vision for the participants, where a rhetorical vision is defined as "a unified putting-together of the various scripts that gives the participants a broader view of things” (Bormann, 1985, p. 133). Conflict emerges from this environment because projects require the participation of numerous groups who have diverse needs represented by contrasting patterns and visions. This was supported by a case study of project management adopted by an IT group in a manufacturing organization. Kendra and Taplin (2004) found that the organization studied had an identifiable culture that existed at the macro level. Their findings also indicated that fragmented sub-cultures existed within the organization, and confirmed that the IT project team represented a distinct sub-culture with shared values and beliefs about the project. Everyone on the project was said to have agreed on the social, technical, and structural aspects of the project. However, there were also individual differences among team members that resulted in conflict in the team. For instance, while some members viewed success as achieving their individual objectives others took a more collective stance and focused on overall project metrics.

In sum, when organizational conflict has been viewed from a cultural lens, it has traditionally emphasized tensions that occur from different underlying assumptions between groups with diverse backgrounds and interests. While such differences are rooted in deep structures of culturally prescribed meanings for individual experiences (Schein, 1992), much of the research on culturally based conflict has attempted to classify types of cultures and establish cultural predispositions reminiscent of the traditional conflict style literature. Although this 
perspective is limited by failing to account for situational and individual differences, it may offer additional insights for understanding diverse belief systems, and open space fo conflict ot have a beneficial impact on individuals whose lives intersect.

Benefits of Conflict. A number of benefits of conflict have been documented in the literature. Conflict has been shown to promote cohesiveness (L.A. Coser, 1956), maintain power balances (Blake, Shepard, \& Mouton, 1964), facilitate change (Darling \& Brownlee, 1984; Litterer, 1966), and promote creative problem-solving (Hall, 1986). From a broader perspective, conflict may have beneficial effects on the organization itself. Litterer (1966) indicated that conflict serves to "initiate a search" (p. 180) for solutions that lead to innovation and change. According to Rubin (1978) "conflict is not only essential to the growth, change, and evolutions of living systems, but it is, as well, a system’s primary defense against stagnation, detachment, entropy, and eventual extinction” (p. 206). This argument is furthered by Putnam (1995) who posits that "conflict is not a breakdown of a cooperative purposeful system. Rather, conflict is central to what an organization is” (p. 184). Thus, conflict among individuals and between groups provides a mechanism to identify and act on diverse interests and goals.

Intergroup conflict has been seen as beneficial due to its role in unifying common beliefs and values (Putnam \& Poole, 1987). For example, in a study of negotiation in teachers' bargaining Putnam (1995) found four categories of benefits from the conflict process. These were “(1) exchanging information and signaling problems, (2) managing differences, (3) balancing power, and (4) promoting flexibility through intergroup memberships” (p. 187). In another study of 105 work groups, Jehn (1995) found a difference in perceived benefit based on the type of conflict that individuals experienced. In this study individuals participated in routine and non-routine tasks. For both task types, relational conflict was always perceived as detrimental to the group. However, task conflict was found to be beneficial, but only for nonroutine tasks. For routine tasks, the participants felt that conflict only interfered with the effective task completion. To foster productive conflict and reduce the potential for its transition into 
personal and relational conflict, some have suggested that organizations should implement outside auditors or utilize “devils advocates” (Cosier, Dalton, \& Taylor, 1991). In a study of multicultural groups Kirchmeyer and Cohen (1992) found that constructive conflict could be fostered through specific strategies such as "searching out a variety of ideas and opinions, fully sharing information, openly confronting differences, and carefully critiquing alternatives” (p. 155). According to the study, such practices resulted in more important and valid assumptions as well as more commitment to the group.

Other benefits of conflict in organizations involve the process of integrative negotiation. This occurs when parties in conflict seek a creative solution which is a "win-win" agreement that benefits both groups (Blake \& Mouton, 1981; Fisher, Ury, \& Patton, 1991). Such active problem solving approaches have often been missed in studies that measured styles in hypothetical conflicts. Van de Vliert, Nauta, Giebels, and Janssen (1999) have proposed a "complexity theory” (p. 476) which asserts that individuals actually exhibit multiple behavioral responses to conflict situations rather than pure styles that have been ubiquitous in the literature. In two studies of a simulated dyadic negotiation, they report that joint effectiveness was improved by a combination of forcing and problem-solving behaviors.

Positive effects of conflict have also been found in new product development teams (Xi, Song, \& Stringfellow, 1998). Conflict emerged as a beneficial element in three aspects of the product development process. First, benefits occurred because new product development requires innovation that is facilitated by surfacing different and contrasting viewpoints. Second, conflict stimulates a more thorough evaluation of alternatives. Third, conflict motivates the mobilization and sharing of information. This follows Baron (1991) who argues that task conflicts encourage new ideas and approaches toward problem solving.

Overall, these benefits from conflict stem from a different focus than provided by the great preponderance of conflict research which asserts that it is a destructive force to be resolved. Because traditional definitions of conflict emphasize the competition for limited resources, this 
view of conflict has emphasized traits and strategies that map the boundaries between self and other. In contrast, studies which identify the benefits of conflict have directed attention toward more interactive and integrative communication behaviors which holistically integrate contextual and relational factors. The present study adopts this latter orientation toward conflict to take a constructionist perspective in understanding conflict and its effects on project teams. To that end, it specifically explores how unique meanings for individual experience can emerge and become enduring as individuals seek out and value information that reinforces their current belief systems while ignoring other equally compelling information.

\section{Constructionist Perspective}

Research on organizational conflict has been criticized for being too positivistic and reductionistic (Nicotera, 1995). Suggesting a constructionist perspective toward conflict, Nicotera argues that conflict is something to be managed, not resolved. Thus, "conflict, if handled appropriately is an important vehicle through which the work of organizations gets accomplished” (p. 4). This follows Butler’s (1973) comments about conflict in projects: “it seems more appropriate for critics to investigate the foundations of perceived conflict with a view to its constructive management rather than its extermination” (p. 86). In this way, managing conflict occurs through communication where communication is understood as the process of developing and negotiating what reality is for specific situations. It is important to understand how this differs from more message-centric views of communication that assumes meaning resides in the messages expressed.

The message-centric view of communication follows the information transmission model (Shannon \& Weaver, 1949) where information is encoded into words and transmitted through a specific medium to a receiver. Potential problems are limited to a few variables such as noise and interference (Littlejohn, 1983). From this perspective, communication can 'break-down' if a message gets distorted by interference. While this model is routinely and ubiquitously used to represent human communication (including the PMBOK), it has serious limitations such as 
failing to account for emergent and dynamic aspects of communication. More importantly, it assumes that only one meaning is present in the message that is open to objective interpretation (Cronen, Pearce, \& Xi, 1990). In contrast, more complex models of human communication posit that communication exists in all forms of social interaction. According to Pearce (1989) "the communication perspective sees all forms of human activity as a recurring, reflexive process in which resources are expressed in practices and in which practices (re)construct resources” (p. 23). This approach argues that communication is not simply a message exchange but a transactional process where meaning emerges and is shaped simultaneously by the participants. According to Shotter (1990) "there are an indefinite number of ways in which the connection between an utterance and its circumstances is, or can literally, be 'made’” (p. 54). Thus, meaning might be said to emerge as individuals construct and negotiate the contours of a specific situation, as they bring different experiences and resources to the conversation, and as they perceive different needs and goals. In this mix, participants attempt to develop commensurable understandings for their common situation. However, because individuals have diverse experiences and backgrounds, the extent to which individual can communicate commensurably is arguably limited, resulting in the potential for conflict.

(In)commensurability. Individuals enter into communication situations with unique combinations of resources which provide them different capacities to participate and contribute. Following Pearce (1989), resources are "the stories, images, symbols, and institutions that persons use to make their world meaningful” (p. 23). The diversity of individuals in terms of age, gender, culture, skills and a host of other characteristics provides the essence of these resources which become particularly important in conflict situations where meaning is being contested. The result of such differences is that the significance of an event and its potential consequences may be invisible to some participants because from their perspective the resulting scenario would not be believable (Perrow, 1984). This suggests that highly diverse groups may be prone to conflict due to their inability to converge on a unity of meaning for the situations they experience. 
The issue of whether a unity of meaning can exist has been well established by the concept of incommensurability (Kuhn, 1996; 2000). Kuhn described human experiences as truly idiosyncratic such that communication is paradigmatic and translation can at its best only approximate the experience of another. Accepting incommensurability as a component of human experience would suggest that a constructionist perspective rather than an objectivist perspective best represent the conceptualization of reality and, therefore, the experience of conflict among individuals. This is supported by Bernstein (1983) in suggesting that conflict is multifaceted and includes incommensurable aspects.

According to Bernstein's (1983) incommensurability thesis, conflict may be thought to occur in three domains representing individual or group differences that are: incompatible, incomparable, and incommensurable. Incompatible differences are those where the participants acknowledge a similar event but disagree as to the appropriate course of action. This follows the traditional definitions of conflict that presume individuals possess the same meaning for the items under consideration, but are in competition for scarce resources. Incomparable differences are those where no basis of logical comparison can be found to adjudicate the viewpoints. In other words, no absolute standard exists that could be objectively applied to resolve the difference. For example, conflict over the "actual” value of an heirloom might be considered an incomparable difference because there is no objective means to establish its value. Finally, incommensurable differences are those where no direct point-by-point comparison can be made because meanings are constructed from fundamentally different ingredients. For these differences, individuals apply completely different meaning systems to comprehend the situation. Thus, participants often rely on multiple comparisons to communicate about these differences and describe their viewpoints. From a constructionist perspective, incomparable and incommensurable differences occur because each individual's available resources and experiences create unique meanings that cannot be precisely understood by others. Consequently, there is no perfect communication of ideas from one person to another (Kuhn, 2000) limiting the ability for individuals to understand and 
resolve their differences. This parallels the schema perspective where conflict results from individuals using different language classification systems to understand and describe their experiences (Pierce, 1979) and follows the Kantian idea that categorization itself constructs meaning (Kant, 1929). Consequently, conflict emerges both from different conceptual meanings and because of incongruent communication about those differences.

This provides the groundwork for the argument that conflict emerges from disparate meanings that individuals construct to make sense of their experiences. These meanings are then communicated in story form to provide plausible explanations for reality. Because, individuals occupy different worlds, their "stories are seldom factual. Instead, stories reflect what people believe should be true” (Hanssen \& Kahnweiler, 1997, p. 120). In this way, individuals reveal their underlying concerns about what is important in what they remember and tell (Schrank, 1990). This is essentially an interpretative paradigm of human communication as it relates to the nature of conflict. As used in the current study interpretive is defined as:

any approach that (1) accepts that knowledge is an inventive, reflexive, socially inscribed symbolic process and (2) seeks to elaborate and enrich understanding rather than establish parsimonious, correspondence validity claims that serve as generalization about prediction and control (Presnell, 1994, p. 18).

This interpretive approach to knowledge is consistent with writings suggesting that what appears as natural in social life is the result of social practices and meaning-making among individuals (Berger \& Luckmann, 1966). Second, it supports the constructionist claims that all knowledge is culturally and historically situated. That is, meaning cannot exist free of context and is constructed from specific experiential elements. This also elevates the importance of memory, emotion, and prior interpretations of events as important constituents in constructing current experience. Third, following Derrida (1988), words themselves have no essential meaning other than what is given to them in a specific context and as negotiated among relevant others. Finally, it suggests that reality is socially constructed through communication, where communication is 
the process of negotiating meanings for their common experiences. Importantly, this process of negotiation does not occur free from the influence of power among the participants.

Organizational power. Because power operates to influence what can be told (Foucault, 1980) participants construct and shape meanings for their audiences through their discourse. Within organizational projects, a structural form of power operates to privilege organizational narratives by providing time and resources to their dispersion. The dominant narrative may be said to represent a discursive formulation (Foucault, 1972) where knowledge and power are intertwined and are constructed together in the official discourse. In this way narratives about technological preferences provide a sense of legitimacy for power to be exercised because it is framed as normative. Within the field of project management, “A PMM [project management model] therefore becomes one of the means by which an organization can create new hegemonic networks of power relations and accountabilities” (Raisanen \& Linde, 2004, p. 103). These researchers argue further that

A project-management model is a socializing force capable of defining, aligning and disaligning heterogeneous actor groups, work tasks, relations and responsibilities. It is a means by which discourse technologists can redesign work processes, turning them into norms, rules and prescriptions, representing established consensual praxis. A PMM is a powerful textual inscription in the 'new' bureaucratization process of multi-project organization, a means of creating a new hegemony by consensus. (p. 118)

This view is consistent with others who have argued that through the discourse of standard project management practices, certifications, and process controls, power has become institutionalized, resulting in an overly deterministic view of technology, which serves to obscure the underlying forces of power to shape project outcomes. Following Orlikowski (1997) "technology is socially constructed by actors through the different meanings they attach to it and the various features they emphasize and use” (p. 406). This parallels Kinsella’s (1999) study of how knowledge was constructed by the scientists in a nuclear fusion research laboratory:

Although scientific consensus has traditionally been viewed in terms of realism, objectivity, and methodical progress toward unitary truth, here I portray 
consensus as a more ambiguous phenomenon embedded in a larger social relations and discursive system (p. 172).

In this case study, the production of knowledge occurred in an atmosphere of high task uncertainty where entailed social power was exercised through normative discipline. "This normative discipline was not imposed coercively or overtly, rather it was a more subtle product of the laboratory discourse and culture” (Kinsella, 1999, p. 173). This culture created an atmosphere where:

Conflicting versions of the facts were reconciled through the production of consensus, a process that was at first resisted by the scientists involved but ultimately accepted under the pressure of a discursive control system embedded in the laboratory culture (p. 173).

However, while the high technology project management environment shares certain elements with a research laboratory, it also differs in several ways. First, project members are temporary members of a team that are pulled from diverse organizational functions and are accountable to different managers. This fosters a climate of mixed loyalties and limited cultural congruence. Second, many project tasks are sequentially performed activities with members entering and leaving the team at different times. Third, team members may only have accountability for their own sub-tasks, and not for the overall success of the project. These factors are posited to create a more equivocal environment with more diffuse and localized expressions of power than described by Kinsella (1999). Consequently, such an environment with diffuse power, ambiguous relationships, and technological uncertainty becomes problematic for individuals to interact and ensure their effectiveness as they collaborate on projects. To better understand how participants resolve these issues and act cooperatively in support of their project goals, the theoretical perspective of sensemaking (Weick, 1995) is provided and explained in detail.

Sensemaking. For the present study, the theoretical perspective of sensemaking (Weick, 1979, 1995, 2001) is presented to model the specific processes that individuals use to construct meaning from their experiences and explain how those meanings may serve to create conflict that is problematic in high technology projects. Sensemaking is defined here as a communication 
theory which describes the process where individuals and groups assign meaning to their organizational experiences by evaluating cues that they have awareness of and perceive as significant in their environments. Awareness and significance of cues represent important individual and group perspectives that are central to how the sensemaking framework informs the role of conflict in organizational projects. Overall, sensemaking is a social process that occurs at individual, group, organizational and environmental levels.

According to Weick (2001) "to make sense of something is to begin to provide a plausible platform for sharing mental models, coordinating activities, and interacting to produce relationships” (p. 95). In this way, sensemaking is about coping with and creating meaning for diverse, contradictory, incomplete, and ambiguous information. Ring and Rands (1989) viewed sensemaking as primarily a mental process that results in a cognitive map, which helps individuals reduce dissonance (Festinger, 1957) from the discrepancy between their beliefs and their experiences. In constrast, Weick $(1995 ; 2001)$ provided examples of how the discomfort with cognitive dissonance is fundamentally a social process that drives the underlying motivation for sensemaking to occur and influences the meanings which emerge.

Thus, sensemaking is more than just interpreting the meaning of an event. While interpretation is a component of sensemaking, the sensemaking construct also involves authoring, creation, and discovery. These elements are contained in the seven distinguishing characteristics of sensemaking as (1) grounded in identity construction, (2) retrospective, (3) enactive of sensible environments, (4) social, (5) ongoing, (6) focused on extracted cues, and (7) driven by plausibility not accuracy (Weick, 1995). These characteristics work together to describe how individuals actively create their experiences in a social environment. Weick (2001) summarized the process as follows:

We could say that when people in an ongoing social setting experience an interruption, they often enact something, retrospectively notice meaningful cues in what they previously enacted, interpret and retain meaningful versions of what the cues mean for their individual and collective identity, and apply or alter 
these plausible meanings in subsequent enactment and retrospective noticing (p. 95).

In this way, individuals are posited to take an active role in assigning meanings to their experiences so as to reduce the perception that their worlds are random and not controllable. Although the sensemaking process is visualized as ongoing because individuals constantly refine their understandings, it also becomes most apparent when people's routines are punctuated by an unexpected interruption.

Typically, the interruption that begins sensemaking is a shock to an otherwise apparently stable system, which has the effect of increasing cognitive dissonance and motivating action (Starbuck \& Milliken, 1988). However, sensemaking can also begin from many small shocks that are barely perceptible in an otherwise stable environment (Weick, 1995). The accumulation of these virtually imperceptible events must be noticed to initiate sensemaking. When a threshold of awareness is reached, individuals label the occurrence which, in turn, signifies that an interruption exists in the ongoing flow of events (Weick, 1995). Once this threshold is reached, people use sensemaking to search for cues that help explain the situation by making connections between individual events. This occurs because people construct interpretations that tend to “emphasize order rather than disorder” (Weick, 2001, p. 36) and have a "preoccupation with central tendencies (the mean) rather than dispersion (variation)” (Weick, 2001, p. 36). This need for order represents an important bias in how knowledge is often constructed from available information. That is, individuals find patterns where they do not exist by focusing on certain existing components and making unconscious inferences (Anderson, 1990). In this way, unrelated events may appear to have associative or causal relationships that form the basis of more global explanations for the unrelated initiating events.

Sensemaking can also emerge when an expected event fails to materialize. Because individuals often exaggerate the orderliness of organizations (Weick, 2001), the lack of an expected occurrence raises ambiguity and creates dissonance much like the emergence of an 
unexpected event. People anticipate organizational patterns and expect specific behaviors to occur. This facilitates a prospective initiation of sensemaking because "as they dwell on what might happen, people’s expectations become better articulated, stronger, and potentially more capable of being a potential force in their own validation” (Weick, 1995, p. 134). Thus, when an expected event then fails to occur, its absence may then become an initiating shock for Sensemaking.

Regardless of how sensemaking begins, the essence of sensemaking is embodied by the principle that individuals construct meaning for an interrupting event by selecting only certain cues from their experiences and ignoring others. Frequently, these cues are expressed as partial explanations or brief story segments which are offered as good reasons for the event to have occurred. Following Boje (1991), "it is not the fact that the story is terse and abbreviated that counts: it is the fact that the teller picks one aspect to abbreviate and another to accentuate (p. 124). Importantly, the construction of story elements is highly sensitive to existing beliefs, which then encourages a self-fulfilling bias toward avoiding even the consideration of other possible explanations. In effect, "to believe is to notice selectively. And to believe is to initiate actions capable of lending substance to that belief” (Weick, 1995, p. 133-134). In this way individuals tend to develop encompassing and enduring explanations for entire sets of organizational activities. As Watzlawick (1976) explained "once a tentative explanation has taken hold of our minds, information to the contrary may produce not corrections but elaborations of the explanation” (p. 50). The elaboration is more efficient and serves to maintain the status-quo. As a consequence, sensemaking in a self-referential environment may tend to short-circuit the corrective feedback system that might otherwise repair misinformation. In other words, one might ordinarily expect that when new information is introduced, mistaken assumptions would be corrected as individuals associate themselves with a more accurate story. However, in a cohesive organizational environment social commitments are a powerful force inducing cognitively consistent behaviors (Weick, 2001). This follows the notion that "the appearance of rational 
action legitimates the organization in the environment it faces, deflects criticism, and ensures a steady flow of resources into the organization” (Weick, 2001, p. 35).

Sensemaking emphasizes that when individuals take action in their organizations they produce part of the environment that they experience (Weick, 1995). Weick defined this phenomena as enactment, in which "people are very much part of their own environments. They act, and in doing so create the materials that become the constraints and opportunities they face" (p. 31). Weick (1995) states that sensemaking is more about a plausible story than an accurate one and emphasizes that "what people keep missing is that what they see is usually the outcome of their own prior actions. What they see is something of their own making” (p. 134). Thus, enactment is a reciprocal structurational-like (Giddens, 1984) activity where individuals act reflexively to (re)produce their environments. The conceptual significance of this is that the enacted environment represents an organizational structure that is constructed rather than reflective of a natural underlying order. This reveals how sensemaking creates meanings that transcend individual messages and reside in broader cultural narratives. It is this "residue of a sensemaking episode that is stored in the retention process as past wisdom” (Weick, 2001, p. 189). This past wisdom is visible in the form of acceptable institutionalized patterns of behavior that are deemed plausible by the organization (Brass \& Burkhardt, 1993). In this way, sensemaking is both an individual and an organizational process that often reifies the established cultural norms and patterns of behavior.

When these strong institutional patterns of acceptable meanings exist in a relatively closed environment, team members may experience "stunted enactment” (Weick, 1979). That is, team members may be embedded within a system of organizational meanings that fail to acknowledge the complexity of the situation and, consequently, rely on existing meanings and interpretations of events (Wright, Manning, Farmer, \& Gilbreath, 2000). This contributes to a “closed-loop quality” (Weick, 2001, p. 180) which is characterized by individuals and 
organizations failing to adequately incorporate disconfirming information and broader environmental information into the sensemaking process.

When individuals make a decision to remain committed to a course of action following an anomalous organizational event, the process of sensemaking may also be employed for postdecision validation (Weick, 2001). That is, individuals may exhibit more confidence and certainty after a decision. Prior to a decision, more attention is paid to the other alternatives than the one eventually selected. However, following a decision, individuals increase their intensity of arguments for the selected path and develop justifications, which also serve to reduce the plausibility of success with the forgone alternatives (Phillips, 2002). This final aspect of sensemaking serves to further decrease dissonance by removing the troubling remnants of alternative choices.

Taken together, these elements of sensemaking help individuals develop a "good story" (Weick, 1995 p. 61). Individuals construct narratives that formulate a reasonable explanation for their positions, attitudes, and behaviors, and justify the actions that they have taken. These narratives are an essential part of sensemaking because they represent both the proving grounds and the showroom for “reality.” Following Wright et al. (2000) "good stories are vehicles that hold together, lend coherence to, and make plausible, the many properties of sensemaking. That is, good stories make good sensemaking.” (p. 815). This aspect acknowledges both the individual and the collective nature of sensemaking. That is, because individuals require social support and share many common experiences, they generate many similar interpretations through a group sensemaking process. Following Freedman and Combs (1996):

People make sense of their lives through stories, both the cultural ones they are born into and the personal narratives they construct in relation to the cultural narratives. In any culture, certain narratives will come to be dominant over other narratives. These dominant narratives will specify the preferred and customary ways of believing and behaving with the particular culture (p. 32).

Weick and Browning (1986) suggest that because organizations are structured more around argument than narrative, individuals often have difficulty with sensemaking beyond a 
limited and localized arena. This is more clearly articulated by Cetina (1981) who comments about how scientific knowledge is constructed in a laboratory environment:

... Products of scientific research are fabricated and negotiated by particular agents at a particular time and place; that these products are carried by the particular interests of these agents, and by local rather than universally valid interpretations (p. 33).

This suggests that attempts at collective sensemaking may prove difficult when power operates to privilege some meanings over others and constrain the cues that are acceptable inputs to the sensemaking process. While the influence of elite group members is seen as providing rules and resources for meaning-making, group members can also interact more democratically to perform sensemaking. For example, Bormann (1985; 1990) demonstrates how groups can develop common narratives through a process of fantasy chaining that creates symbolic convergence among group participants. When this occurs, individuals add their own story segments to a narrative being told or influence its telling by challenging particular aspects. It becomes a group narrative as individuals converge on a story that is acceptable to the group. Importantly, this convergence of meaning then becomes an organizational resource for important organizational activities and future sensemaking processes as the participants seek relevant cues from their environment.

The cross-functional and segmented structure of projects requires participants to occupy different organizational sub-cultures, have access to different resources, share limited time together, and develop mixed loyalties. The complexity of this environment has been described as creating a natural propensity for conflict to arise and potentially affect the success of projects (Pinto \& Roughiainen, 2001). In addition, project teams are often faced with aggressive goals to optimize cost, time, and quality objectives with limited resources and unknown risks. This scenario is posited to contribute to the formation of conflict as multiple meanings emerge for project ambiguities that encourage team members to become committed to different and perhaps incompatible courses of action. The current study will seek to find examples and explore the 
nature of such conflict where discordant sensemaking is evidenced by incongruous meanings and enacted with counterproductive results.

\section{Summary}

This study has reviewed the relevant literature on organizational conflict and demonstrated its theoretically functional foundations. Primarily, conflict has been viewed as a trait characteristic, and artifact of competence, a consequence of cultural differences, or as a style of behavior (Blake \& Mouton, 1964; Kilmann \& Thomas, 1975; Putnam \& Wilson, 1982). Each of these approaches has primarily positioned conflict in deterministic terms and has resulted in reductionistic strategies emphasizing communication solutions based on sending and receiving appropriate messages. This has shaped organizational assumptions and expectations about how individuals can effectively function to accomplish institutional objectives. For example, some have suggested that project managers have different personality traits from functional managers that make them successful in their individual roles (Ives, 2005). Others provide training to improve communication by active listening and other techniques to avoid relational conflict. Overall, much of the research in organizational conflict has adopted some variation of Thomas and Kilmann's concept of conflict styles. The consequence of this has been a narrow focus on the cognitive rather than interactional modes of conflict. For instance, the emphasis on styles has encouraged a limited definition of conflict effectiveness with corresponding strategies toward making a correct assessment of the appropriate response (Conrad, 1991; Putnam \& Poole, 1987). In addition, the findings from this limited approach have been far from deterministic.

Equivocality in conflict findings may be associated with the research assumption that complex contextual variables can be reduced to a few salient factors that predict effective strategies or with management biases that direct research for personnel use rather than for construct exploration (Burrell, Buzzanell, \& McMillan, 1992, p. 121).

For these reasons, the current study has brought forth and incorporated an interpretive approach in conjunction with a sensemaking framework to broaden the understanding of conflict and its impact in organizations. 
The current study has applied this framework specifically to high technology projects for the purpose of investigating how conflict may operate as a precursor indicative of project failure. As detailed in Chapter 1, high technology project failures are both chronic and costly problems that continue to occur at alarming rates. In addressing high technology projects, the current study follows research arguing that the challenges of project management are not technical, but behavioral and organizational (Belassi \& Tukel, 1996; Thamhain, 2004). In addition, it responds to the limitations in much of the prior research that has framed project success primarily in terms of project manager characteristics and ignored the specific project characteristics, team member characteristics, and factors external to the project (Belassi \& Tukel, 1996). As a result, the current study strives to include a more holistic account of the project environment as it is experienced by the participants during important decisions and activities that influence project outcomes.

The current study begins with investigating high technology projects from the perspective of the project manager who is the central figure held accountable for project success. Projects will include those that were considered successful and unsuccessful as well as how the criteria for success were established and may have changed throughout the project. The focus of the study will be to uncover how sensemaking among stakeholders result in conflicts that when left unabated are indicative of project outcomes. To ensure a more complete understanding of this process, data will also be obtained from other stakeholders such as team members, project sponsors, and end users. Because perceptions can then become self-fulfilling prophesies as individuals enact their beliefs (Weick, 1995), the study will emphasize the connection between project conditions and the decisions and behaviors that were a direct consequence of participant beliefs. To that end, the research questions are designed to develop a richer understanding of how enduring conflict is indicative of project outcomes and how it occurs through the sensemaking process in high technology project teams. 


\section{Research Questions}

In selecting high technology projects and focusing on the sensemaking perceptions of the participants, the intention of this study is to better understand how conflict emerges and becomes enduring in projects and how its consequences may be reflective of project outcomes. Its interpretive constructionist approach has followed some recent project management research in arguing for the importance of context in understanding project failures (Hallgren \& MaaninenOlsson, 2005; Ives, 2005; Jaafari, 2003). Although Jaafari posits that both project and environmental complexity are significant factors leading to failure, the role of context has yet to be fully explored. For the present study, contextual factors are of interest because of their potential to create disparate sensemaking cues for individuals in different roles or who have different project interests. In that regard, the first research question explores the role of project context by addressing how it contributes to conflict.

RQ1: How do project members experience complexity as a factor in project conflict?

One aspect of project context which has been frequently cited is ambiguity over the criteria for project success. And, because the present study seeks to understand how conflict may be a precursor to project failure, it is important to also understand how projects are deemed successful by the participants. If differences emerge for the definition of project success or evolve throughout projects, these differences may have important consequences for how the participants communicate and coordinate their project plans. Therefore, the second research question is:

RQ2: How do the participants of high technology projects understand project success? 
Since conflict is usually envisioned as a unitary interpersonal and intraorganizational phenomena in which individuals vie for limited resources, this traditional perspective fails to address how different meanings may emerge and be open for contest. In addition, traditionally functionalist approaches can underemphasize the role of the project environment as well as the emergent nature of high technology projects. From this broader perspective, it is plausible that multiple forms of conflict may exist within the project and affect its outcomes. Therefore, the third research question is:

RQ3: What are the significant types of conflict that exist and become enduring in high technology projects?

Finally, to directly address the primary focus of the current study, the fourth research question explores the connection between enduring conflict in projects and project outcomes. That is, it seeks to reveal how ongoing conflict in its various forms can act as a precursor to project outcomes. It does so from the cumulative findings of the other research questions, which establish the types of conflict experienced in projects and how sensemaking works within actual project environments for individuals to construct meanings from their project experiences and take actions which influence project outcomes. Therefore, the final research question is:

RQ4: What is the role of conflict as a precursor to project outcomes?

Taken together, these research questions establish the basis to explore high technology project problems from a new perspective. That is, these questions work to uncover how different forms of conflict may emerge and become problematic as individuals use sensemaking to generate distinctive meanings and act in service of their beliefs about their project experiences. In this way, constitutive elements leading to project failure may be better understood. As 
Pentland (1999) indicated in citing Simon (1992) "as we move from our surface observations toward the underlying structure, we move from description to explanation” (p. 712). Thus, a more thorough understanding of how conflict may emerge in high technology projects and have meaningful consequences may contribute to more robust explanations for project outcomes. To that end, the current study invokes a qualitative methodology to answer the research questions in this study. 


\section{CHAPTER 3}

\section{Methodology}

The present study utilized an interpretative constructionist framework to understand the experiences of individuals on project teams especially as they perceived how conflict became problematic in their projects' eventual completion. This methodological approach supports the ability for researchers to uncover the contextually and culturally specific meanings that subjects hold for their experiences and communication behaviors. This is particularly important in the present study because its primary purpose is to examine how conflict emerges in specific project situations and may have important consequences in project outcomes.

To achieve this goal, the present study utilized participants who are actual members of project teams and have participated in the completion of multiple high technology projects. Investigating the experiences of individuals in actual projects is important because it addresses limitations frequently found in laboratory studies. Such problems include the use of subjects who have no prior or expected future relationship with one another, experimental outcomes that are inconsequential for people's lives, and a general absence of contextual relevance. In addition, examples from Bowers (1973) as cited in Schneider (1987) argue that natural groups overcome three characteristics of laboratory studies, which inappropriately inflate their claims to validity:

1. Experimenters play with treatment conditions until they have their desired effects.

2. Random assignment of participants to treatments fails to account for the tendency of humans to routinely select themselves into and out of settings.

3. Persons have an active role in creating their own environments as much as environments shape peoples' reactions.

These issues are found in much of the research on conflict topics, which has been frequently based on hypothetical and artificially controlled conflicts (W. A. Donohue, 1981; Putnam \& Jones, 1982). While such studies allow control over a number of variables, they also 
fail to incorporate important relational factors. For example, many of these studies are designed to have the participants only meet once and for a limited time. These individuals frequently have no history together prior to the experiment and are likely to not meet again following the experiment. The participants also have no choice in selecting their interaction partners. Therefore, it is difficult for experimental laboratory studies to replicate actual experiences that subjects encounter in their own ongoing group environments.

In contrast, the qualitative approach utilized in the present study opens space for understanding the unique perspectives of the participants. Qualitative methods can accomplish this because they primarily (1) are concerned with process rather than outcomes, (2) focus on meaning as understood by the participants, (3) incorporate the researcher as the essential instrument, (4) involve fieldwork to access and include the participants' environment, (5) orient toward the description rather than the cause of behaviors, and (6) are inductive, beginning with details of experience and working toward broader concepts (Creswell, 1997). In this way, qualitative methods support the goals of this study, which were to understand the participants’ experiences of conflict and its consequences from their own perspectives. Two methods were used in support of this goal and data from each served to provide more depth and breadth to the overall findings. The first method was participant observation applied to the researcher's own experiences in meetings and events of the Project Management Institute.

\section{Participant Observation}

The method of participant observation provided the opportunity to become familiar with and develop an in-depth understanding of current project management topics as understood by the practitioners of project management from a large number of organizations. As an active member of the Project Management Institute and by attending numerous chapter events, I met with over 100 project managers at 14 local chapter meetings in three states. The meeting format was typically structured to include time for networking and personal discussion followed by a 30 to 45 minute presentation. Typically, these were dinner meetings where additional opportunities were 
present for individuals to share their experiences and learn from one-another. The presentations were classified as officially sanctioned training sessions and the members could receive training credits for their attendance.

From my participation in these meetings and post-meeting discussions, I accumulated approximately 60 pages of field notes, which were primarily centered on the experiences and stories that project managers told about their own projects. In addition, most of the presenters made their power point slides available on the internet so that the meeting participants could avoid note taking during the presentations. In all, I downloaded over 200 presentation slides and these became additional research artifacts to reinforce the field notes. Many of these slides included speaker notes, which further clarified the intended meaning of the slides. The invited speakers were most frequently project managers or sponsors, but also included consultants, academic researchers and others who addressed current topics in project management. The focus of most presentations was on helping project managers be successful in their projects. Consequently, the presentations were highly practitioner-oriented and often provided detailed guidance for topics such as risk management, project planning, software support tools and the like.

The data from the participant observation research was categorized by topic and reviewed as it was collected. I attempted to transcribe the notes within 24 hours, which allowed me to provide a more structured format and add my impressions of my observation experiences. In addition, I referenced the presentation slides that were associated with each meeting. This proved useful because the informal conversations following each presentation often included references to particular aspects of the presentation. Later, during the final analysis, these participant observation transcripts were included with the interview transcripts to construct a more holistic picture of the topics being researched. The interviews were conducted in parallel with the meeting participation and included some of the same individuals. Because the interview method was the primary method of data collection for this study it is discussed next in significant detail. 


\section{Interviews}

The primary method for collecting data in the current study was conducting interviews with project team members and related stakeholders. This required a process of identifying the research participants who would have access to the relevant topics of the study and be accessible to the researcher. Thus, a plan was developed and implemented to acquire an appropriate constellation of participants for interviewing.

\section{Interview Method}

To obtain appropriate interview participants, the current study utilized the technique of non-random purposeful selection, which is traditionally used in qualitative research (Creswell, 1997). Following Holstein and Gubrium (1995), "the idea is not so much to capture a representative segment of the population as it is to continuously solicit and analyze representative horizons of meaning” (p. 74). These researchers define horizons of meaning as the contours of experience that occur for a particular area of human behavior. This suggests that researchers should purposely identify and solicit input from individuals who are not only prototypical participants in the specific topic of study, but also those who are less centric, and yet, still have important insights based on their more peripheral involvement. This was achieved in the present study by involving other project stakeholders, such as project sponsors, customers, and team members. For example, one participant was the director of the organization's program management office, who had the responsibility to set the standards for and oversee the operation of all projects within the organization. In that role, he was more interested in significant budgetary and legal issues that may arise from project activities, rather than how individual projects functioned. While he had little direct knowledge of project operation, he was an important informant regarding the senior management's perspective on overall project operation and success. He also participated in employee review and promotion activities for each of the project managers in his organization. Thus, individuals were selected for research subjects based 
on their unique standpoints and became informants to these experiences. This approach is supported by Warren (2001) who argued that:

because the object of qualitative interviewing is to discern meaningful patterns within thick descriptions, researchers may try to minimize or maximize differences among respondents - say, according to race or class - in order to highlight or contrast patterns (p. 87).

For the present study, participants were selected who were core members of high technology projects as well as more peripheral stakeholders. In addition to software and I.T. projects, the participants also represented other types of technology innovation such as electronic and medical products. Since many studies on high technology project management have been limited to software related projects, this broader focus was intended to potentially uncover differences that may be characteristic of other technologies. The variety of projects represented in this sample is shown in Table 7.

Table 7 - Participants by Project Type

\begin{tabular}{ll}
\hline Project Types & Number of participants \\
\hline Enterprise software & 11 \\
Telecommunications & 3 \\
Medical Devices & 2 \\
Computer hardware & 4 \\
Electronic security products & 2 \\
Navigation instrumentation & 2 \\
Nuclear physics & 1 \\
Industrial controls & 4 \\
Process implementation (ISO-9001) & 1 \\
Other & 6 \\
\hline
\end{tabular}


For access to participants, I used prior business and personal contacts to identify a few initial participants. Then, I utilized a snowball sampling technique, where some participants that I interviewed provided the names of other potential participants. A second method was to identify potential participants through my participation in the professional chapter of the Project Management Institute (PMI). Prior to this research project I was an active member of the PMI and frequently attended meetings and events. Thus, the final sample included PMI members from three different states and four different local chapters.

\section{Interview Participants}

The research participants in this study were professionally employed individuals, over the age of 21, who were willing to be interviewed relating to their participation in an organizational project. These included both team members and project managers who were responsible for project outcomes as well as other stakeholders, such as project sponsors, customers, and support staff. Because individuals are often involved in more than one project, the interviews inquired about multiple projects and responsibilities. I choose project managers with a variety of skills and organizational backgrounds and only selected those who had at least five years of experience and make their primary income from their work with project teams. This level of experience was requested to ensure that the participants could contrast their own experiences and possibly provide richer descriptions of unusual occurrences. In some cases I interviewed more than one member of the same organization when the situation became available and it appeared that doing so provided valuable information in support of the study. However, the research questions do not require information from multiple participants on the same projects because the goal is to better understand how perceived conflict among individual team members was indicative of project outcomes.

To begin the study, I focused on project managers, because they are held accountable for project success and their role is most centric to the project activities that occur. This also helped establish how role differences could become important mediators of meaning for project activities 
and helped determine who the other participants should be. This became important, because I quickly determined that team members were often transient participants and frequently had a limited vision of the project outside of their own contribution. Consequently, I began to include those who occupied levels of responsibility higher than or equal to project managers and who also could influence the project outcomes. However, these individuals who were primarily project sponsors or program managers offered little about specific project activities. Consistent with their role, these participants were more helpful in their overviews of problematic practices and organizational procedures. Two of the participants were the clients of large IT implementation projects and provided insights from their perspective as both team members and the beneficiaries of their projects.

In total, for the interview method, there were 36 participants who reported on 48 projects. Of the 36 participants, 21 (58\%) were male. While five participants declined to reveal their age, the remaining 31 ranged in age from 32 to 61, with a mean age of 44. Four participants were project sponsors or management personnel, five had only been team members and the remaining 27 were project managers. The final number of research participants was determined by “theoretical saturation” (Lindlof \& Taylor, 2002, p. 224), which is the point that additional data failed to provide new insights to the research questions. Theoretical saturation is a commonly accepted technique in qualitative analyses to determine the appropriate sample size. Because data were transcribed and tentatively coded following each interview, saturation became clear prior to the last three interviews (which were all project managers).

\section{Interview Philosophy and Process}

Because qualitative research is less familiar to many researchers than quantitative methods, the nature of interviews in qualitative research has frequently been misunderstood. Following Seidman (1998):

The purpose of in-depth interviewing is not to get answers to questions, nor to test hypotheses, and not to "evaluate" as the term is normally used. At the root 
of in-depth interviewing is an interest in understanding the experience of other people and the meaning they make of that experience (p. 3).

Therefore, the methodological focus applied to the interviews in this study is to help individuals articulate the meanings that they have constructed for their project experiences with emphasis on how project conflicts may have emerged and become problematic to their success. To accomplish these objectives, the current study follows Holstein and Gubrium’s (1995) constructionist approach to interview data. Their concept of the active interview suggests that every occasion for communication has the potential to change the facts and data that are produced. Holstein and Gubrium contrast the active interview with conventional interview approaches where the subjects are conceived as "vessels of answers” (p. 7) and "repositories of facts” (p. 8). Such a traditional perspective assumes that through carefully worded and emotionally neutral questions, the subjects' answers can be provided uncontaminated by outside influences. To ensure this, other procedures are used such as attempting to create question neutrality or asking the same number of questions on each side of an issue. Presumably, the careful use of these methods will produce the "correct” interpretation of data.

In contrast, the objective of an active interview "is not to dictate interpretation but to provide an environment conducive to the production of the range and complexity of meanings that address relevant issues, and not confined by predetermined agendas” (Holstein \& Gubrium, 1995, p. 17). Therefore, the interview process was loosely structured with questions that oriented the participants to the topics of interest. This process consisted of one or two interviews with each participant. The interview was focused on the background and overall experiences of the participants in regard to their perceptions of project conflict and its apparent consequences. Once the basic demographic and background factors were understood, I began following the interview guide in Appendix A. In one case a second interview was conducted at the request of the participant who, upon reflection, wanted to share more detailed and in-depth examples of his project experiences. 
Each interview session was approximately one-half hour to one hour in length and was conducted at a location convenient to the participant. In some cases this was the business office of the participant and in others a local coffee shop was selected. Three of the interviews were by phone at the request of the participants. The interviews were audiotaped with the knowledge and consent of the participants. Backup taping equipment including spare batteries and tapes was available if needed. The tapes were confidential and no names were recorded on the tape. All tapes are stored in a locked cabinet in accordance with the storage requirements outlined by the University of Missouri Institutional Review Board. The interviewer also took notes with the permission of the participant. The interview process began with a brief explanation of the study and included informed consent. Then brief demographic data were collected for the purpose of better understanding the study's participants. An interview guide was used to provide an organized but loosely structured approach toward accessing the experiences of the participants. Interview Guide and Techniques

The active interview process utilized in the present study is consistent with the constructionist approach to knowledge described previously. The primary role of these questions was to stimulate thematic and dynamic involvement of the subject on the research topic (Kvale, 1996). As such, it is inherently analytic along with being informative. For these active interviews, an interview guide is primarily "advisory, more of a conversational agenda than a procedural directive” (Holstein \& Gubrium, 1995, p. 76) indicating that its use will vary from interview to interview based on the unfolding interaction patterns. With these limitations stated, the interview guide is shown in Appendix A.

Beyond the questions themselves, the active interview process includes the purposeful dialogue of the researcher as an important aspect of the analysis (Freedman \& Combs, 1996). Rather than being concerned that the interviewer may distort the answers, the active approach coconstructs the interview data by using techniques that probe and even challenge the statements of the subject (Holstein \& Gubrium, 1995). Unlike positivistic interviews where the interviewers 
are concerned with contaminating the otherwise pure vessel of answers, the active interview asserts that knowledge revealed through the contribution of both participants in an energetic dialog. "The active view eschews the image of the vessel waiting to be tapped in favor of the notion that the subject's interpretive capabilities must be activated, stimulated, and cultivated” (Holstein \& Gubrium, 1995, p. 17). Thus, the goal of interview analysis is to uncover the "range and complexity of meanings” (p. 17) that subjects can generate from their experiences. In this way the subjects are viewed as constructors of meaning and organizers of experience. The rationale for this approach can be found in the objections voiced during traditional interviews. For example,

It is a common experience for investigators to craft interview questions carefully, only to have participants respond with lengthy accounts, long stories that on the surface have little to do with the questions. ... Subsequently, I realized that participants were resisting our efforts to fragment their experiences into thematic (codable) categories - our attempts, in effect to control meaning (Riessman, 2001, p. 695).

Such extraneous comments are often treated as unwanted artifacts in traditional interviews, but when viewed from a constructionist perspective, call attention to important individual meanings that may be otherwise lost. To accomplish the active interview, a number of authors have developed useful techniques to engage subjects, which are seen as helpful in creating the dialog that reveals participant experiences. The specific methods selected for use in the current study are described in Table 8 along with a brief explanation and referenced sources.

Table 8 - Interview /Analysis Techniques

\begin{tabular}{lll}
\hline Technique & Explanation & Reference page \\
\hline Variability and Contradiction & $\begin{array}{l}\text { Interviewer notes and may call attention } \\
\text { to variations and contradictions in the } \\
\text { subject's narratives to gain understanding } \\
\text { and explore the connections among } \\
\text { events. }\end{array}$ & $\begin{array}{l}\text { (Holstein \& Gubrium, } \\
\text { 1995, p. 32) }\end{array}$ \\
& $\begin{array}{l}\text { The interviewer explores a discrepancy by } \\
\text { challenging the plausibility of a claim in a } \\
\text { tentative, non-aggressive, and non- }\end{array}$ & $\begin{array}{l}\text { (Evans, Hearn, } \\
\text { Uhlemann, \& Ivey, } \\
\text { Confrontation }\end{array}$ \\
& &
\end{tabular}


judgmental manner.

Narrative Incitement \& Narrative Precedent

Introducing Specific Language

Contextual Shifts and Reflection

Uncovering Alternate Linkages

Promoting Multivocality

Sensitizing Concepts

Typification

Enlisting Cooperation
The interviewer actively provides precedent and may incite specific narratives through giving examples or asking for stories about specific topics.

The interviewer may introduce specific language and terminology to probe for meanings and notes the specific language usage by the subject.

The interviewer notes the subject's use of context and actively probes for the aspects of context that affect the situation.

The interviewer explores how the topic discussed might have alternate linkages to other events and probes for the horizons of meaning that are perceived by the subject.

The interviewer explores the complexity and range of meanings that the subject creates through suggesting the subject take different points of view, adopting the positions of others.

The interviewer searches for and explores the assumptions and issues that are held to be important by the participants.

The interviewer searches for and explores the categories that individuals assign to their experiences. This may include both prototypical and extreme instantiations that serve to establish categorical boundaries.

The interviewer solicits help from the participants in clarifying the meaning for their experiences.
(Holstein \& Gubrium, 1995, p. 39)

(Holstein \& Gubrium, 1995, p. 49)

(Holstein \& Gubrium, 1995, p. 55)

(Holstein \& Gubrium, 1995, p. 58)

(Holstein \& Gubrium, 1995, p. 77)

(Sass, 1994, p. 150)

(Freedman \& Combs, 1996, p. 24)

(Evans et al., 1998, p. 219)

These techniques were applied in an ad hoc fashion depending on how the interviewer perceived the information would fit within a plausible and holistic account of the events and how comfortable the subject appeared with the interaction. The nature of these techniques is 
decidedly constructive and stems from the underlying goal of exploring the depth and complexity of participant experiences. These techniques proved particularly effective as several participants initially offered clearly ambiguous and contradictory information about their experiences. Then, through techniques such as confrontation and variability and contradiction, the participants were probed for more in-depth explanations. In several cases this led to the surfacing of underlying assumptions about how project and organizational activities should occur. Thus, through these active interview techniques, the researcher was able to gain better access to the participants' own experiences and the influences of project context and environment as the participants perceived their effects on the projects. Following the interviews, further analysis and interpretation were conducted using a thematic post hoc analysis.

Post hoc Analysis and Interpretation

The interpretive interview positions participants as narrative resources who are better visualized as stocks of knowledge than as a vessel of answers (Holstein \& Gubrium, 1995). While the 'vessel of answers' approach presumes that fixed answers reside in the minds of the participants that must be carefully extracted to avoid biased data, the 'stocks of knowledge' concept suggests that specific instantiations of knowledge can emerge within an interview, which are tied to the subject's individual experiences and how they make sense of the information along with the researcher. Following the interview, data were assessed for the dynamic interrelatedness of what was described and how it became meaningful. Special attention was given to chronology and consistency markers that indicated how meaning evolved throughout the project. To accomplish this, the present study explored chronological accounts with thematic analysis to assess meanings developed by the participants. The topic of project management is a good candidate for this approach because projects are organized chronologically and have identifiable events that are usually marked by participants with respect to the project timeline. It was sometimes particularly productive to have the participants describe their project experiences chronologically with the project timeline. Themes that emerged were deemed important when 
they were described by multiple participants. In this way, confidence is developed that the specific experience may also occur in projects outside of the study. This propensity is enhanced by utilizing participants from varied organizations and who represent diverse project types.

Thematic analyses have been found to represent ways in which individuals interpret differences in their meanings in specific contexts and uncover the understandings they hold (J. T. Wood, Dendy, Dordek, Germany, \& Varallo, 1994). Thematic analysis not only reveals categories of experience, but also can illuminate:

patterns and meanings that are not visible from within a relationship, are most likely perceived when outsiders' perspectives are also voiced. Researchers who are not caught up in the inner logic of relationships and who are informed by theoretical and conceptual matters, have the capacity to situate local lives within larger settings whose structures and processes infuse particular partnerships (J. T. Wood et al., 1994, p. 132).

The basic thematic approach follows the work of Owen (1984), who indicated that an important theme may be indicated by any of three criteria. First, if a topic reoccurs. That is, the same meaning occurs at least twice in a thread of conversation. Second, repetition establishes a theme. This criterion requires specific key words to be repeated, indicating their significance in the conversation. Finally, the criterion of forcefulness indicates that a specific word or phrase occupies an important role for the speaker. This characteristic may be detected by paraverbal characteristics of the speaker, such as volume, intonation, or inflection. Thus, the application of thematic analysis is not to generate deterministic relationships but rather to map the contours of the participants' experiences.

Beyond the specific themes which emerge, this analysis also focused on the holistic aspects of the narratives that were co-constructed during the interviews themselves. The interactions between interviewer and participants produced thick-rich descriptions (Gertz, 1973) that are indicative of the participants' experiences from their actual environments. For the present study, the story components are particularly important in applying sensemaking (Weick, 1995) as the theoretical framework with which to understand how individuals experience project 
events and develop their own perspectives. As previously described, sensemaking is about developing plausible rather than accurate descriptions of events, and then are used by participants in justification of their actions. Consequently, the analysis of interview data was particularly sensitive to the sensemaking actions of the participants. To achieve this, I coded individual thematic elements on index cards, which were referenced with a code number for the interview. I used single phrase descriptors to best represent each thematic concept. For example, one of the common themes was "surprised by the customer's interpretation of a requirement". By using the traditional qualitative techniques of open coding and axial coding, I formed preliminary categories and then used constant comparison to re-entered the coding process from the beginning each time the categories were altered. Eventually, I converged on the final categories described in Table 9.

In sum, the active approach to interviewing requires the interviewer to "furnish precedence, incitement, restraint, and perspective” (Holstein \& Gubrium, 1995, p. 76) and engage the participants in lively dialogs for the purpose of uncovering their comprehensive accounts of experience. Therefore, it is a conversation with a guiding purpose and a plan where the concern for contamination is replaced with awareness for the idiosyncratic construction of knowledge. Analytic Tools

The active interview process is complex for a number of reasons. First, the role of the interviewer becomes an important aspect of the data. The responses of the subject may take on special meaning that is more accurately understood when it is directly tied to the prior comments of the interviewer. Second, there is the potential for multiple voices to be coded. For example, it may be informative to distinguish between a subject speaking as a team member versus his or her interests as a stockholder. In this way, one person may represent multiple subjects providing different nuances of meaning (Holstein \& Gubrium, 1995). Finally, a number of narrative artifacts such as framing or defensiveness may represent conversationally important cues for characterizing the nature of the commentary. Therefore, it is important to adequately manage a 
substantial body of data and include these considerations in the analysis. This process begins with transcribing the researcher’s notes and tape recorded interviews.

Transcription. The audio tape-recorded conversations were transcribed for analysis, resulting in 452 pages of single-spaced text. Identifying information was omitted during the transcribing process. Potentially identifying words were replaced by four x’s [xxxx] and sometimes followed by the word "name" or "organization" when needed for clarification. When necessary for privacy, phrases were redacted and noted appropriately. Interviewer notes were entered into the transcribed document and designated as such. Notes were added to indicate responses that may have been transparent to the audio tape. These included notes and sufficient transcription symbols to reflect the speakers' thematic emphasis. For example, loud voices were represented by all capital letters. Also, certain behaviors such as using hands to clarify meanings were coded in brackets. For instance, one participant routinely talked about levels of the organization as “up here” by placing her hand horizontally above her head. This was coded in brackets as [motions higher with hands]. Pauses and other subtle linguistic artifacts were coded only when they represented an exceptional and unusual aspect of the interview that appeared to be vital to understanding the intended meanings. Once transcribed, each interview was maintained in a separate data file that identified the participant by a code number.

\section{Verification}

Most business research has been conducted under a logical positivist or functional paradigm using quantitative statistical methods where reliability and validity are carefully defined terms that provide a uniform method for assessing confidence in the results. Under the functional paradigm interview reliability would determine the extent to which each question would generate consistent answers. Tests for validity would ensure that the interview questions actually measured what they intended to measure. However, the interview data gathered in this study is assessed from a constructionist perspective where reliability and validity are defined differently. Following Holstein and Gubrium (1995): 
When the interview is viewed as a dynamic, meaning-making occasion, however, different criteria apply, centered on how meaning is constructed, the circumstances of construction, and the meaningful linkages that are assembled for the occasion. ... One cannot expect answers on one occasion to replicate those on another because they emerge from different circumstances of production. Similarly, the validity of answers derives not from their correspondence to meanings held within the respondent but from their ability to convey situated experiential realities in terms that are locally comprehensible. (p. 9)

Thus, the objective of the constructionst or 'active' interview is not to "dictate interpretation, but to provide an environment conducive to the production of the range and complexity of meanings that address relevant issues, and not be confined to predetermined agendas” (p. 17). In this way, confidence in the analysis and interpretation of qualitative data is traditionally obtained by verification.

Verification is the process of examining data for the purpose of gaining confidence in its accuracy and has been accepted as a standard of quality in qualitative research (Creswell, 1997). For the present study, verification is provided in three ways. First, multiple sources of data were used. The interview method provided direct access to 36 participants who reported directly on their own experiences in projects. Except for two cases, each of the participants worked for different organizations and participated in different projects. In addition, the projects studied represented different types of businesses and technological issues. These projects included software, hardware, medical, industrial, and business process objectives. Additionally, the participants were located and worked in five different states. Thus, the themes that were consistently found across this wide variety of participants provide confidence in their reports.

Second, a participant observation method was used where the researcher attended numerous meetings of local Project Management Chapters in four different communities and three states. The documentation from this method included field notes and presentation slides to support the researcher experiences. While a small number of participants were common to both methods, this was primarily an independent source of data and used to reinforce the findings of the interview data. 
Third, a member check procedure was implemented to gain confidence that the researcher's conclusions are coherent representations of the participants' experiences. Eighteen of the 36 interview participants (50\% of the participants) were randomly selected and contacted to review their transcripts and selected research notes about their data, to determine if the researcher’s understandings were accurately represented. Incongruous differences were not found during the procedure, so no additional action was deemed necessary. However, one member insisted on changing her quotation to add additional information and remove one phrase that she described as sounding overly critical of her employer. Since this quotation was not used in the study, the changes were not problematic to the results. Another subject requested and was granted a second interview to add additional experiences which he deemed important to be represented in the study. He was quite prolific during the second interview which consumed over an hour. Overall, the member checking procedure provided participants with the opportunity to assess the degree to which the results actually represent their experiences. Their feedback was then used as evidence that their experiences were accurately represented in the interpretations and conclusions of the study.

Overall, the three verification methods work together to provide evidence and build confidence in the actual data reported in the study and, more importantly, how that the data are understood and assessed accurately by the researcher. Because this data and some of its verification involved contacting human subjects, the research was conducted under the auspices of an Institutional Review Board.

\section{Institutional Review Board}

For research involving human subjects, The University of Missouri - Institutional Review Board (IRB) requires submitting certain project documentation for review and obtaining researcher training prior to conducting research. I met those requirements and obtained prior approval for conducting this study. In addition, the interview guide and the consent form (included in Appendix A and Appendix B, respectively) were provided as required. 


\section{CHAPTER 4}

\section{Results}

The primary focus of the present study was to investigate the role of conflict as a precursor to high technology projects that failed to either partially or completely meet their objectives. In some cases, this meant that the project was canceled altogether, but more often projects were partially completed with less functionality, more cost, and/or later than originally intended in the project charter. Prior literature has suggested that high technology project failures have less to do with technology problems than a myriad of ongoing management and communication efforts that affect discretionary project orientation, activities, and decisionmaking. For example, Jaafari (2003) has argued that

The prevalence of failure currently experienced on many technology-based projects has been studied widely and found to be generally related to managerial approaches to human and organizational factors, rather than technology, per se (p. 53).

In that regard, the present study has used a qualitative approach to interview project managers and team members about their experiences on high technology projects that have experienced some degree of failure. The research participants were also asked about and often volunteered important information about their successful projects and contrasted those projects with their less successful ones. Because I primarily interviewed experienced project managers and team members, their insights were often reflective of numerous projects, which then became particularly helpful in documenting those differences and mapping the communication, activities, and events that were perceived as early indications of pending project failures. To provide a more comprehensive understanding of projects and how they operate in organizations, I also acted as a participant observer in professional meetings and events held by the Project Management Institute. Through these experiences, data were collected that provided additional breadth and depth for the topics addressed in the current study. 
In support of the first two research questions, the interview and participant observation research began with a concentration on the contextual and environmental factors relevant to projects and their outcomes as perceived by the participants of the study. The two topics of project complexity and the ambiguity of success became ubiquitous early in the data analysis and were pervasive themes throughout the research.

\section{Project Complexity and Success Criteria}

For the present study, the participants attributed numerous project related problems to the existence of project complexity. As they described project complexity, the term generally corresponded to the quantity and variety of external factors which were perceived to affect their projects, making them more difficult and potentially affecting their outcomes. For the most part, these were non-technical and non-task factors relating to the project itself, its personnel, its sponsoring organization, or its environment. These influences could combine in different ways to become problematic anytime throughout the project. Similarly, the criteria for project success were surprisingly ambiguous and often evolved as projects proceeded along their course. These two factors were consistent with the research described earlier that has suggested both have considerable effects in projects. Therefore, these topics were investigated further in answering the first two research questions of this study.

\section{Project complexity}

Project complexity is often equated with task complexity (PMBOK), indicating that problems arise from the management of a large quantity of tasks, highly interrelated tasks, or tasks that are difficult to complete. An underlying assumption for this traditional understanding is that projects are generally isolated from their environment, self-sufficient in terms of resources, and that the requirements are well specified and relatively stable. A vastly different picture emerged from the participants of the current study who described how both organizational and environmental issues were primarily what made their projects complex and problematic. They 
described several different types of project complexity along with examples of how it became problematic in their projects.

Member diversity. First, membership diversity was considered a significant form of complexity that could create project problems, especially at the later stages of projects where individuals were described as more interdependent. The actual project members were often said to be a diversity of individuals with different needs and perspectives, who interacted in unique ways to have considerable influence in their projects. For instance, some members came and went throughout the projects, some were customer representatives, and others were third party contractors who were actually employees of a third organization. One project manager working on an IT project in a public utility described some of their vendors as team members who became like employees, yet retained separate interests.

P: On big projects we will bring in vendors that have expertise in that application, and say “ok, let's work with you.” And they will have people on site, and they'll be here for a couple of years, whatever it takes to do it. Their folks will come in. After a while, we can't tell if they are contractors or employees because they've been here so long. They have a badge but they're not required to wear it. It's not uncommon for contractors to be on site for 3-4-5 years. And they will work with their company and be the liaison between us.

I: Does that cause any conflicts?

P: I would say that at the project management level where the project manager is torn between the company he works for and say "ok, if you want to add this requirement, how do I help the company I work for and give them, bring in revenue" versus "this is my customer and I want to do a quality job for them."

In this case, the supplier of a large software product provided technical resources to help customize and implement their product for this public utility. Part of this process was to loan out their employees as "contractors" to the client organization to assist in customizing the software. Clearly the contractors and client's own employees had somewhat different agendas, which were reported to create problems for the project when contractors took actions that were for the benefit of their own organization at the expense of their client. Such an arrangement creates an interesting boundary problem for the participants from the standpoint of conflicts of interest. 
That is, the organizations and their individual employees have both cooperative and competing goals to manage without clear boundaries regarding how that should be accomplished. The participants reported being concerned about this ambiguity and the uncertainty it produced in these situations, especially in regard to sharing and withholding information. Yet, these situations continued because there was no apparent solution.

Customer involvement. A second type of project complexity was represented by customer involvement on project teams. This is a common occurrence for organizations that offer enterprise software (software that operates across an organization's entire business network). Because of the complexity and cross-functional impact of these systems and the tremendous impact on the client organization, these software implementation projects will often involve a strong contingent of customer representatives. Typically, these participants are much more than observers and have important responsibilities that may include contributing to project specifications, functional priorities, and module testing. However, customer involvement also adds a variety of complexities. For example, organizations have little control over the behavior of their customer team members and may struggle to get the needed attention and cooperation from them. One project manager for this type of organization described her difficulties with simply getting customers to read the project requirements document:

P: ...I then sit down with my customer face to face and we actually walk through the SOW [statement of work] section by section. We read it together. I provide it to them in advance. Ask them to read it, typically they don't. And in the meeting I read it to them, and that's where we match up our expectations. Most of the time, the customer and I see eye to eye. But the challenge is to get their time and their attention, to get them to actually read the SOW and think about the impact it will have on their own organization. I can't make them do that, but I try.

In this example, the participant clearly indicated a history of frustration in trying to get customers to read and respond to the statement of work. The difficulty in completing this first step raised anxiety and ambiguity about the customer's commitment to fulfill their ongoing obligations as part of the project team. This makes the project more complex to the extent that the project 
manager felt obligated to seek out additional commitments and assurances for customer participation as well as more clarification of the proposed requirements and timeline. This is based on the premise that customers have, at least to some degree, different viewpoints, values, and cultural assumptions than the project organization that is responsible for delivering the needed product. When such differences are not fully understood, the gap may be a potential source of problems. Consequently, the ambiguity between the parties may produce a perceived need for additional risk assessments or contingency planning.

Another related complication of customer involvement is their level of willingness to make their project commitments a priority. Since customer team members typically have other primary responsibilities, several project managers complained the customer members were unreliable in fulfilling project tasks. For example,

P: Often a customer will not free their technician up from their other responsibilities. They'll tell them, well you also have project xyz and then the poor technician has to figure out, "do I work extra hours, do I give less priority to my current work, how do I' balance this" and it's not unusual for a technician to decide the $\mathrm{xxxx}$ project is less important than keeping those servers running.

Variations of this theme were commonly expressed by the participants. IT implementation projects often had customer members who were assigned to participate and, yet they were not relieved of other duties. However, since these participants were clients of the software provider, there were sensitive relational issues making it difficult for the project manager to hold the client members accountable for their full contribution. That is, the project managers understood how important it was not to offend or embarrass their clients, especially because the client possesses final project sign-off authority, which could be easily withheld or delayed.

Multiple customers. A third type of complexity reported by the participants was the existence of multiple customers for a single project. Typically, the customer is described as a monolithic entity that holds a privileged position as the funder of the project who is often the ultimate arbiter of success as well as the primary beneficiary of the project. With this model, there is a single point of contact to arbitrate customer requirements and acceptance. However, 
several of the projects in the present study had multiple customers who were in conflict among themselves or at least had competing interests in the project. This is exemplified in a large hardware and software development project where an air traffic control system was being developed for both a civilian and a military customer.

P: Our company had the contract for the xxxx [country] air traffic control system, for the civilian system, and then the military was responsible for terminal control around their air bases, so they wanted a system that would be compatible with the new civilian system and could be integrated with theirs.

I: I see.

P: So what we were doing is we were taking the terminal control part of the civilian system and modifying it for the specific military requirements.

I: OK...

P: All we did were the modifications that we felt were needed. The Air Force guys viewed it that they had a stand alone specification. And therefore, if something got changed on the civilian side, we still had to provide a, you know say a capability got deleted on the civilian side that we still had to provide it to them on the military side. And that is what happened because we were totally dependent on the work that was being done for the civilian system which was of course, ah twenty times larger system than the military one was.

In this example, the program manager was caught between the conflicting needs of two

customers. At the beginning of the project, the needs were aligned and both customers were contributing resources relative to their expected benefits. However, the project became more complex during the development phase when the larger customer needed to reduce costs, which they attempted to accomplish by reducing features. Unfortunately, some of these were needed by the military customers, resulting in an ongoing conflict between the two customers, creating significant complexity in having to manage a new and evolving mix of features and costs.

Organizational intrusion. A final and perhaps the most significant type of project complexity discussed by the participants was the intrusion of the organization that sponsors the project. While the idealized model of a matrix-management organization allows for a strong and independent project team with self-sufficient resources, seldom were projects described to actually exist in that form. In many cases, projects were interfered with by 'outsiders' in the 
organization who were seeking to promote their own agendas and had the power to affect the project or its outcome. For example, in one project referred to earlier, a corporate vice president had his own competing project and used his influence to place roadblocks in the path of the project team. In other cases, changing goals or business conditions within the organization altered project resources or priorities. Further, additional complexity was sometimes generated when projects were delayed substantially beyond their original targets. Such delays were reported to result in regulatory changes, technological obsolescence and the like which were not likely to have occurred within the original time frame. For the present study, the primary impact of project complexity was its introduction of additional ambiguity and uncertainty, with the result of making routine project communication and decision-making more difficult. With more ambiguity, sensemaking (Weick, 1995) became more difficult for the participants as they struggled over different interpretations for relevant events and the optimum responses. Overall, the numerous sources of project complexity created a more ambiguous than certain project environment which contributed to a dynamic and evolving definition of project success. Thus, along with complexity, the concept of success became problematic in these high technology projects.

\section{Project Success}

While the criteria for success were often formally documented at the outset, success in actuality was almost always more pliable and uncertain as the project progressed. The PMBOK (PMI, 2005a) describes a process where the success criteria for a project are well documented and measured against a baseline schedule that provides the means to report discrepancies affecting key project metrics such as time, budget, or functionality. However, projects seldom followed such static processes and the concept of success was often fluid throughout the project cycle. Several organizations that had formal project definitions and statements of work for their projects effectively ignored them or so frequently and drastically modified them, that their value became more symbolic than functional. In other cases, major project changes were made without updating the documents at all. For example, 
P: No sooner did we have the project requirements signed off, the project sponsor told us to make a major change to the project. But the sign-off and approval process took so long, with so much red tape, that there was no way he was going to go through that again. He just said we're going to add this other feature, but we're not going to go back and change the project. Just work it in.

I: So were the change and the project successful?

P: Well, which project are you asking about? We were funded with resources for plan A and then, plop, we were working with plan B. If you mean did the plan B work? - eventually. We made it work, but it cost a lot more, -- it took almost 8 months longer because we had to completely redesign the control circuitry and purchase new test equipment and there was a lot more testing involved.

I: So if it was 8 months late and more expensive, how could it be called a success?

P: Because, in reality, it was a different project. If it sells, people quickly forget about what the rules were. And it's like when the dust settles, there were so many undocumented changes, that nobody could really go back and say "why did you do this?”, - as long as it works.

Here project success was handled on a month to month basis and according to the project manager, the organization was tolerant of delays because it was driven by a powerful sponsor, it was a new technology, and the team members also had other project assignments. The participant went on to describe that because there was enough ambiguity among projects and personnel, the project sponsor could bury the excessive costs in other projects and hide the changes until the project was complete. Once it was successful, the changes and delays were justified by the outcome. In this way, the static concept of success was subverted by an organizational culture that valued results over process and vested the power in key individuals to simply ignore the official processes. While this approach was not sanctioned in writing, the project members understood that success could be defined by those in authority. These ad hoc managerial styles were often reported by project members who worked for small high technology firms.

One characteristic of the high technology business environment has been the emergence of many small entrepreneurial companies. These organizations often have engineer-owners who work side by side with their technical staffs developing new products. The next example was one such company where owners selected projects based on their own technology interests and would 
engage a project team to make it a successful product. However, for this project, the criteria for success were poorly defined.

P: But now if we are looking from the technical aspect of it, it was just, it was obviously a company initiated project, it was not built around an RFQ [request for proposal]; it was built around something the company felt like was a good and potentially profitable product, and ah but it definitely started off poorly.

I OK., so you were saying earlier about it starting on a whim. Was there a project plan or some sort of document that would say this is what is going to be done?

P: There was some but it was certainly very skimpy and there was certainly no clear cut product definition to guide us as to what we were trying to produce or what was set to goals. There was certainly no specifications written for the receiver and the transmitter that expected performance of the transponder as a whole.

This participant recalled how there was no customer request [RFQ] for this product and no technical requirements that were being designed around. Therefore, it was difficult to tell when the prototypes were working well enough to say they were successful. The project continued on in an incremental fashion with additional design improvements, and never reaching a finished stage. Because the product was very expensive and the production units were built by engineering as one-at-a-time prototypes, there was no natural transition point that might otherwise mark the end of a design project. That is, there was no need to transfer the project into a production environment, which would typically indicate the completion of a design project and an official recognition of its successful functionality. For this organization, project success was often declared by an owner when he grew tired of a project and had it working well enough to abandon it for another adventure. Although the company was growing and new employees were attempting to create more standard processes, the project developers were often left wondering about how to declare success or when to admit failure when the owner lost interest in it.

This ambiguity of success criteria also applied to software projects in a somewhat different form. That is, software developers exercised a number of options for declaring success of a project and shipping it to customers. Two of the most frequent practices reported by 
participants involved labeling the operational status of particular features. The first technique was how phased releases could be utilized to redefine when a project was complete. Essentially, to make some of the projects successful, the project team would sometimes delay features that were planned for the product. To accomplish this, some of the lower priority features would be moved to a future version when the completion date was perceived to be at risk. This practice provided a great deal of flexibility for declaring success in IT projects, especially when problematic features were peripheral to the central functionality of the project. Because of this, several software project managers revealed that their projects would almost never be considered failures.

A second but related aspect of software project success ambiguity is the ubiquitous use of "release notes.” The participants indicated that most of their software products were shipped with a readme file which was ostensibly used to provide clarifying information about setup and operating procedures of the software, especially in regard to specific implementations and hardware compatibilities. However, some participants reported using the readme file extensively to describe problems that could not be fixed by the required release date. In this way, projects could be labeled as successful, even though a number of features were only partially functional.

In the following example, a project team was responsible for developing a ruggedized industrial computer, which used a combination of custom and standardized components. The team was responsible for the basic hardware design and wrote Linux compatible machine level software drivers to handle a variety of optional boards.

I: So what happened then [when the project was delayed]?

P: Well, there was something screwy about the $\mathrm{xxxx}$ video card, and we could never get it to work reliably in its highest resolution mode. It looked like a shared memory conflict of some sort. But it was intermittent - really hard to find. So we tried everything and spent a huge amount of time working with the manufacturer trying to figure out -- is it our hardware or software, or their card? We never could get it working and it was delaying the release of our computer.

I: So what did you do then? 
P: Our management decided we couldn't spend any longer on this problem, so we just documented it in the release notes as an incompatible board at that resolution, and bingo, our product is ready to ship.

I: And putting it in the release notes made it ok?

P: We call it the graveyard of hidden problems.

This is consistent with the comments of a senior program manager from a large software supplier who indicated that their software products often required three releases to make all of the originally intended features perform to the original SOW. However, even then, this manager indicated that users report many more defects in each release than were ever found in testing. Several participants indicated that this has become a part of the software culture. That is, it has become accepted that software is unlike any other product and not every feature will work right out of the box. But loyal customers were said to endure through the extended period of upgrades and future releases to help make the end result appear 'successful.'

Another problem with clearly understanding success is when the scope of work is highly ambiguous or continuously changing. While this may be perceived as a problem that occurs in small organizations, one of the largest projects in this study was also plagued with this issue. In this project, the consultant revealed that in this ongoing billion dollar project, there is no SOW.

P: You can walk around the building and ask pretty much anyone who are now PMPs, and probably all of them except maybe two or three, and I think there's 7 to 9 of us. And if you ask them if they do project management 101, just basic project management, you'll get a rousing NO. In fact, you may even get a HELL no. Cause they don't do it, they don't do it.

Here, when the participant lamented about the lack of basic project management she was referring to how the participants could not track the basic deliverables or intervene to stay on schedule because there was baseline to measure against and the tasks themselves were constantly changing. She includes all of this in her discussion about the scope of work.

I: So what are the major gaps?

P: I think the biggest gaps are again, the first thing you do on a project is set up your scope of work. You ask somebody, what's your scope of work, 
well, we have this document. It defines what success is. Ok, well, what's the document about? "I don’t know, figure it out.” [almost sarcastically]

I: So you have this huge project, and you still don't have a good scope of work?

P: No. If we had a good scope of work, ok, we wouldn't be having two hundred change proposals a year. So right there that tells you. One of the first things I did [after being hired], because I figured they had to have a change log. I said, "let me see your change log." And this was March. They already had from Sept. to March, ninety three change proposals. So what do you deduct? You don't have a scope of work. You have no clue what your scope of work is. You have no clue when you're done - where the endpoint is.

In this project, the document that was referred to was a multi-volume set of project plans, but specific timelines and task descriptions were clearly outdated and there were hundreds of addenda that were constantly requiring the redeployment of team members to different tasks. This is an example of very large projects where contractors obtain revenue from "earned value”³ by documenting the completion of substantial sub-tasks, regardless of the status or eventual success of the overall project. According to this participant, it was advantageous to the contractor to have ambiguous scope and task requirements because each task could result in a new discovery that might require additional work. Because of the project's highly technical nature, only the contractor had the expertise to assess the results of each task and then recommend the best course of action. Consequently, these project managers administrated the tasks but could not quantify the scope of work or identify the criteria for success.

In a final example of success ambiguity, two organizations had conflicting views about the functionality of a multi-million dollar electronic navigation system. The contract called for the design and development of two independent systems with the intent of providing redundant operation in case of a system failure. In fact, a major part of the contract was to specify the guaranteed reliability of each system. Only when the systems were designed, delivered, and subsequently failed, did the companies realize that they had conflicting interpretations for who would have final system integration responsibility. 
I: One thing I am still confused about is what were the expectations regarding success for this project?

P: It was interesting, because as individual systems, the equipment worked pretty much like we said, and we had a formal customer acceptance test where the customer bought off on the systems. But those two systems were never designed to work together.

So, when the system got installed and it was not working, our company had the position that it was the customer's responsibility to make them work together, to integrate them. But the customer took the position that they were sold the equipment specifically as two systems working together in the same environment. They felt like integration was our job.

In fact, it ultimately became a real debate between the lawyers about the word "independent" and what it meant. The contract requirement from the survey company was to ensure location accuracy by providing position information from "two independent systems." Our interpretation of the word "independent" was that the systems would be independently tested and then become the responsibility of the customer. But, the customer's interpretation was that they were independent in terms of their requirement for redundant functionality, but were a single system in terms of operation.

This participant indicated that his organization met each of the literal contract requirements but admitted that the product was virtually unusable in the field. Later, the survey company purchased other equipment and there were lawsuits resulting in an undisclosed settlement. In this case there was an arguable difference between user interpretations of needs and supplier obligations, resulting in two incompatible criteria for success. This example reveals how even a clearly defined and stable view of success became ambiguous when accounting for an incompatible interpretation from the external customer. In this way, it differs from the previous examples but emphasizes the role of communication in establishing the context for project outcomes to be judged successful.

\section{Summary}

These examples have portrayed some of the many ways that issues of project complexity and project success are interrelated and perhaps mutually constituted. These findings illustrate how both issues have the capacity to become problematic as they increase the ambiguity and uncertainty in high technology projects and, consequently, enhance the likelihood for participants 
to engage in more incongruous sensemaking. Such differences create an atmosphere for the creation of conflict and the potential for it to become enduring as individuals create meanings for their actions and subsequently make behavioral commitments to those meanings (Weick, 2001). Thus, from the sensemaking perspective, the emergence and endurance of conflict have important project meanings and consequences.

\section{Conflict Types}

While project complexity and the variability of success criteria were found to be enduring themes for the projects studied, these topics were significant in the current study because of their relation to the construction of conflict and its effects in project teams. When the participants were asked about their project experiences, they routinely described both successful and unsuccessful projects. Frequently, they volunteered their insights and were enthusiastic about contrasting those differences. When asked about conflict, they commonly understood conflict as loud or angry communication exchanges about differences of opinion on a particular topic. Several participants also equated conflict with blame directed toward a team member for not fulfilling a project task obligation or a manager for not providing the necessary time or resources. Because these conceptualizations of conflict were much narrower than the current study intended, I found it useful to probe for other project experiences that would fit the broader definition of conflict embraced by the present study. To that end, I asked about differences in expectations among the participants or between the team and the customer, which may have become problematic for the project over time. Since expectations might be implicit and difficult for the participants to articulate, I also asked about surprises that they had experienced, which led to significant project consequences. This line of questions was particularly fruitful, as most participants could easily recall at least one or two meaningful surprises that they experienced during their projects. Finally, I inquired more directly about how the teams communicated, made decisions, and solved problems, with the intent that the participants might reveal forms of conflict in these activities. While several themes emerged from these data, the most obvious was that 
three distinctly different types of conflict were experienced consistently by the participants. While each type was conceptually distinct, they were practically enmeshed and related, and in many cases one type evolved into another. Therefore, it is useful to briefly review each type before describing how they were reported by the participants.

Unexposed conflict is characterized by issues relating to adequate knowledge and consensus of the most central concerns of a particular project. In its more latent form, important project information held by one stakeholder was not communicated at all to other members. However, this type of conflict also included differences that were discussed but where the parties held incongruent meanings for significant project issues. These situations can be described as conflict because actual and important differences exist that will become apparent later in the project. In addition, some participants also reported struggling with their perceptions that there could be important unrecognized differences between themselves and their partners. While such differences remain unexposed, their detrimental impact may increase because individuals are not exploring and addressing such differences. This category was most commonly represented by difficulties in (a) the ability for project teams to identify client expectations and needs, (b) understanding team capabilities and limitations, and (c) assessing needed and available resources.

Unexplored conflict centers on issues of power and voice, where project success may be impeded by organizational forces operating within and outside of the official project charter. Because these conflicts represent issues that cannot be addressed openly, participants may struggle to identify the sources and boundaries of power that influence their projects. These typically fell into three areas (a) the ability for project teams to surface organizational imperatives and assumptions, (b) the existence of competing interests, and difficulty in (c) coping with sources of power and control.

Unresolved conflict is represented by ongoing issues of action and timing, particularly related to group decisions and activities. These conflicts were characterized by groups having difficulty resolving disagreements and disputes that were openly discussed and debated. The 
three common issues described for this type of conflict, were problems with (a) adapting to changes, (b) agreeing on priorities and scope, and (c) negotiating solutions to recognized problems.

These three conflict types and their major constituents are shown in Table 9.

Table 9 - Conflict types and categories

Unexposed Conflicts (Issues with knowledge and ambiguity)

(1a) Identifying client expectations and needs

(1b) Understanding team capabilities and limitations

(1c) Assessing needed and available resources

Unexplored Conflicts (Issues with power and voice)

(2a) Surfacing organizational imperatives and assumptions

(2b) Uncovering competing interests

(2c) Coping with veiled sources of power and control

\section{Unresolved Conflicts (Issues with action and timing)}

(3a) Adapting to change

(3b) Agreeing on priorities and scope

(3c) Negotiating solutions

While the participants frequently attributed project failure to one or more of these issues, there were several examples of the participants describing how overcoming one of the specific problems resulted in the project being saved or "turned-around." In addition, the participants often volunteered their contrasting experiences on successful and unsuccessful projects and reflected on those differences. This led to an increasing emphasis during data collection on asking more follow-up probes contrasting successful and unsuccessful projects. Therefore, the results along with some of the examples include both positive and negative consequences based on how these types of conflict were managed. 
At the outset, it should be emphasized that it was not just the existence of conflict that appeared to have seriously problematic consequences in these projects. Rather, it was also how these conflicts were indicative of important underlying differences that became enduring and interfered with desired project outcomes. In this way, the emergence of conflict was the indicator of important differences and protracted conflict revealed how those differences became enduring, possibly generating the momentum for increasingly negative project outcomes. This potential was seen for each of the three types of conflicts that were discovered in this study. While each of these is discussed separately, it should be noted that they often occurred simultaneously.

\section{Unexposed Conflicts}

As previously defined, unexposed conflicts represent issues with knowledge and uncertainty that, when left unabated, may become problematic to project success. The participants were consistent in reporting three significant categories for this type of conflict. They represented difficulties in (a) identifying client expectations and needs, (b) understanding team member capabilities and limitations, and (c) assessing needed and available resources. Each of these is discussed separately below.

Identifying client expectations and needs. From a consensus of project literature, one of the key criteria attributed to project success has been a clear statement of work often represented by a customer requirements document. Since the customers or clients of a project are the primary beneficiaries, their expectations and needs ideally represent the core elements and objectives for the statement of work. However, this idea of comprehensively communicating with customers to capture their expectations and needs was substantially problematic in the current study. In some cases, there was virtually no communication at all, which created serious problems when those differences became known. However, there were also cases when the project manager communicated frequently and built substantial relationships with the clients, and then was very surprised when conflicts emerged that revealed significant differences between the parties regarding the project requirements. For example: 
I: When would you say was the first indication of a problem?

P: I was sitting in a meeting with about 5 end users, and what I had, I had established a relationship in purchasing, that's going to be my advocate. I established project advocates in each one of my end user departments. So I had purchasing, I had warehouse, I had order entry.

And I am sitting in a meeting with about 5 of these end user advocates. And they're talking and we had just gone through a run through the prototype, and nobody was happy and everybody was saying "this doesn't do what I thought it would do and I'm uncomfortable telling my manager that you know we'll be able to use this."

And I just in my head I'm seeing red flags popping up all over and I'm thinking well how did this happen? What's going on here? So it was really in that meeting that I started to realize that I was in trouble. I wasn't able to for sure identify why I was in trouble, because I just didn't have enough experience. What I did know was my product wasn't being accepted by the end user.

The project manager for this project had spent significant time developing relationships with numerous individuals from the various user groups within the organization, yet was totally surprised when the users revealed different meanings for how they thought the software would perform. For this example, it was not a deficiency in the amount of communication but rather how the participants constructed meanings and made sense of the information available to them. The end user advocates had knowledge about their own requirements and interpreted ambiguous information from the project manager as favoring their needs. Similarly, the project manager interpreted the acceptance of the advocates as evidence that she was on track with the appropriate design. In this way, sensemaking (Weick, 1995) worked at the individual level and tended to reinforce the existing beliefs of all parties, which greatly reduced the opportunity for their differences to be identified early in the project.

In other projects, communication to gather requirements was virtually absent altogether. While the idea of thoroughly gathering requirements from end users is so basic that some of the participants in the study referred to it as "project management 101,” it was surprising how frequently this issue arose in these projects. However, for some projects without a specific targeted customer, this was more difficult than it might otherwise appear. For research and 
development projects, there often was no specific customer identified for the product until after it was developed. As such, organizations and their project teams only had indirect information about what features the end users would need and value. In some cases, this information was reported to originate with sales and marketing departments which often relied on matching the features of similar but competing products that were currently in the market. In other cases, high technology companies were driven by the technology itself without sufficient regard for the business profitability. For example, one company chose to develop a new product based on the desire to participate in an emerging technology without assessing whether the customer base could afford the product. This participant was a project manager representing a team of successful investors who completely failed to understand their potential customers. He reported that,

$\mathrm{P}$ : By the time I was brought in, the contract for the $\mathrm{xxxx}$ units had already been signed. And they were really proud of the price that they could get for the large quantities that they agreed to. So it was like a 10 year contract, and they got the xxxx units at such an unbelievably low price, that everybody had stars in their eyes. The supplier was jumping through hoops because they had a high volume production stream for 10 years, and these guys were going to make millions, and bla bla bla.

\section{I: So what went wrong?}

P: Well, a lot of it [the reason for the failure] was that they hadn't done the market research. They were attempting to sell a Cadillac to a market where every Chinese knock-off is being sold on the web, and if you check on the web you can get the product for $\$ 99$ that they were trying to sell for $\$ 400$. And how do you show someone that this one is worth another $\$ 300$, because of its features? But they don't perceive that value at all.

In this application, the target market was traditional college students who are well known for limited financial resources. Yet, the project sponsors had little comprehension for the cost impact of their product on this audience and how their potential customers might be making sense differently regarding the cost and utility of this project in contrast to their other alternatives. Rather than attempt to diagnose how the students made sense of the product, the sponsors invested additional funds in advertising to persuade the students of the value. This further 
marketing failed to convince the students that the extra cost offered any substantial benefits, and the company rapidly terminated their operations.

Another example of not identifying customer needs involved a small engineeringoriented organization of about 100 employees. The owners would initiate projects based on their desire to incorporate new technology rather than the customer demand for a specific product. Therefore, the intended customers were not contacted or consulted about the product until the prototype was functioning, and only then when the team was attempting to test it. In one particular project, this organization developed a very innovative and expensive product without understanding who would buy it.

P: ... and another problem that the project team encountered but this was much later toward the end of the project was something that should have been foreseen early on is that the customer base for it really wasn't there. And there was little research done into what the customer base would be and to determine that as it turned out there were very few people, very few like $\operatorname{xxxx}$ out there who had the money to spend on an expensive system. But that was discovered after the project was virtually completed and they were looking for customers for people to buy the product and there were very few that could lay out that kind of money... And that should have been discovered early on. I mean there should have been somebody asking the question who is going to buy this system, what we think it is going to sell for, does this customer base that we anticipate have the money to buy this kind of equipment and the answer probably would have been a little scary had they done the analysis.

The two participants who reported on this project indicated that there was great pride in the technical ability of the team to create this product and offer it to the market. Although small, the organization was quite compartmentalized, which made it sensible to the engineer/owners that the problem of selling it was only a "marketing problem,” which was perceived to be minor in comparison to the technological innovation required to invent it. Within this context, the inventors failed to value important cues relating to customer affordability in deference to their pride in achieving a technological innovation. In addition, they held an underlying belief that new technology would create new customers, although they had not identified where this market would come from for the project that they were undertaking. 
While this was a small organization making a sizable investment without identifying their customer needs, larger organizations ran projects with similar profiles. In the following case, a telecommunication company was developing an electronic product called a 'switch' which is the central control element in telephone systems to route data and conversations from one place to another. This organization ran into similar problems:

P: I've been in telecom for several years, and the company that I worked for would say we're going to build this fancy switch and it's going to be the best thing since sliced bread and they wound up spending tons and tons of money and they got this wonderfully working product at the end and nobody wanted to buy it.

I: How does something like that happen?

P: Just from not having a clear vision of where they were trying to get to, like I said, they had all kinds of money, they pumped into the thing and everything went beautifully, it wound up finished on time, and there was just no market for what they were trying to build.

I: So tell me more about what happened at the end of the project.

P: Well, I guess the marketing group believed they could sell a whole bunch of these things if only we could build it. And when it came right down to it, as we got closer, they started forming strategic alliances with all of these other telecom companies in an effort to partner, and when it came right down to it, they just never sold anything. They would ship some out as demos or whatever to show the stuff off, and show how wonderful it was, but nobody came to spend any money-- when it came to spending money, nobody wanted to buy them.

The participant reporting on this project was a software developer who had little awareness of the customer requirements or business partnerships related to this project. This type of product was designed with industry standard specifications to be interoperable among the competitive systems operating within the United States. However, because of this, there was no specific customer for the product, making the assessment of needed features more ambiguous. According to the participant, in addition to the standard requirements, each of the competitive products being developed had a unique combination of additional features whose value had to be determined in the marketplace. Ultimately, although this product technically met its functional objectives and was delivered on time, it failed to gain market acceptance and, thus, failed. Without a production 
contract with a major telecommunication provider, this company that invested most of its assets in this new design, laid off their employees and went out of business. The participants provided many examples like these where major projects were undertaken without understanding the value of specific features from the customers' perspectives. It is a common oversimplification to treat this as simply not gathering requirements. Rather, the sensemaking perspective of the sponsoring organization frequently introduced substantial bias in the interpretation of customer needs based on their own needs. This confusing of perspectives was a major sensemaking problem in adequately identifying customer requirements.

There were many reasons given for not acquiring better feedback from customers or getting more accurate information about needed product features. Often the participants indicated that it was deemed too difficult or expensive to gain access to their customers or that the communication was filtered and of little use by the time it reached the technical team. However, even for internal projects, where the end users are part of the same organizations, project leaders sometimes failed to obtain critical information about customer needs. For example, this project manager was responsible for implementing an IT project throughout an organization and found problems in both the functionality and the technological approach.

P: We had collected requirements from approximately $75 \%$ of the people who were going to be affected. We hadn't even identified everybody who would be touched by this. I mean there were departments we didn't even know about that had to be involved. So we hadn't appropriately identified all of our constituents. We hadn't collected requirements from them. The technology that we were embarking on using was right at the end of its life cycle. We were building something on a mainframe when the client-server was about, I don't know this was in the 1990s, so client-server was fairly mature then, and we were still on an old mainframe with green screen terminals. So we had obsolete technology. What, I began to realize -- I was in a lot of trouble when my end users were coming back we had started doing some very early prototyping and end users were giving us a lot of “this doesn’t work for me," "this isn’t working,” and "I can’t use this.”

This project manager attributed her inexperience to not identifying and seeking additional input from each of the stakeholders. Unfortunately, when this project manager learned more about the user needs, it was at the end of the project and the type of changes needed were so significant that 
little could be done to save the project. This was typical of several project examples where the critical information that could have saved a project was discovered too late to alter its outcome.

A number of project managers identified the step of obtaining comprehensive customer requirements as their most important task. One incorporated what he claimed to be a particularly successful approach, which was to allow the customer to establish the requirements. In this project, the definition document detailed the project requirements and the test criteria that would need to be satisfied for the project to be considered successful.

P: I had a project for the design of a railroad control system. The customer actually wrote what we called a definition document. They told us just exactly what it was that they wanted. And we would base the project requirements on their definition document.

I: And what impact did that have?

P: You wind up with a very satisfied customer. One downside of doing a project that way is that a significant way into the project, they will want to come back and say "well we want to make this one little change to do this instead of that." They feel it is their right to do that. And you opened the door by letting them define the requirements in the first place. But still, the feedback there is so immediate and so direct from the end customer that unless you're just incompetent, you'll have to end up with a happy customer doing it that way.

This project involved the integration of hardware and software to provide very complex

operational features that had to work as a subset of a larger system for a specific customer. Thus, obtaining the precise design requirements was particularly important for this application. By having the customer actually define the requirements in his own language, the project team was able to further engage the customer with conversations to effectively translate the customer's language into their own project requirements. This might be described as a type of 'forced collective sensemaking' because the project manager was responsible for integrating the project definition document with the other project documents. In completing that process, he was forced to clarify several meanings that the customer had articulated in the document. This revealed numerous differences that were resolved before the project progressed. While much of the success was attributed to this process, it also created another issue. That is, the end product was 
also intended for other customers, so the risks in letting one customer specify its entire functionality is to possibly miss other important and potentially contradictory needs. This illustrates how sensemaking is directly related to the overall context of the project and its larger environment.

Some of the IT project managers emphasized the value of early project communication to clarify requirements and found that the adoption of early prototyping provided an effective means to accomplish this. One participant described the practice of using simulated software displays before the software application is actually developed. These screens provide what appear to be actual results to the end user for their review. This participant reported that the rapid prototyping technique represented better communication by confirming the client's needs in a very concrete fashion.

I: So, help me understand this gap in capturing the requirements. You said the user knows what they want... where's the gap?

$\mathrm{P}$ : It's conveying the information to the programmer or to and from the team. I think that's why rapid prototyping is a pretty nice thing to do, Saying here's this, is this the direction we want to go. "Yes." Ok, then the next generation we will add features A, B, C. Ok, being able to do that, and build upon it, and it gives quick success.

I: So is the idea of rapid prototyping the fact that, does that mitigate or solve the problem of "what is the requirement?"

P: Yea, because what it does is puts a tighter requirement on it. And you can say, we're going to start with this one program ... and if that works, we'll go out to the next level. Getting success in small ways is important, because their requirements are going to grow as the project grows too. "Oh, we need a report to do this too." And, "this data here you need to expand a little bit.”

This participant was a PMP certified, contract IT project manager who routinely worked for a number of clients providing customized financial software. In his view, software providers were constantly at risk of providing software that would be unacceptable to clients. Therefore, he indicated that his strongest tool was to generate rapid prototyping screens and do so in small increments. In this way, customer communication was frequent and centered on the actual 
project outcomes as provided by simulated displays. This is another technique that creates 'forced sensemaking' and is ideal for software projects because the user interface represents the actual underlying required functionality and is easy to simulate and deliver to the end user for feedback and evaluation. However, for hardware projects, this technique was described to be less effective. Models and prototypes were only approximations of the needed features, requiring communication to be much more explicit and include additional details about intended functionality and performance.

Understanding team member capabilities and limitations. In addition to conflicts that emerged from a lack of identifying customer needs and expectations, a second category of unexposed conflicts centered on problems with understanding the capabilities and limitations of the project members themselves. The participants frequently reported conflict among the team members and stakeholders of projects based on their capabilities and limitations of performing the tasks that they were assigned. The structural backdrop for this was reflected in two terms that were most frequently used for people by the study participants. Project team members were routinely referred to as "resources” and "skill sets.” In some software organizations, the participants reported that team members were identified primarily by their skill set rather than their names or titles to facilitate their ability to be substituted for one another on projects. In this way, managers who needed a "skill set” could shop around for "it” and interchange people as needed to keep their projects on track. However, while this may have seemed like the ideal language for assigning personnel and optimizing project outcomes, participants indicated that this approach often created problems by failing to identify their unique needs and abilities. Perhaps more significantly, the participants reported that individuals were frequently required to perform other tasks that were not adequately factored into overall availability. For example, in the following project, a project manager needed time from a person who was a member of the client organization. However, she described continuing problems in trying to gain time commitments for an employee who had other high priority commitments that limited his availability. 
I: How do you determine if you can get time from a team member in the customer's organization?

P: What I do is ask the customer. I anticipate I will need Joe Jones and by phase I will need him full time here, I'm going to need him about half time here, and then for training, for two weeks, I'll need him full time again. Are you able to give me Joe full time for that period of time? Because if you can't we might not be as successful as you want to be. Most of the time the customer, the guy's manager or executive will say "sure no problem." Where we get into trouble is where the rubber meets the road and Joe finds out what his executive has committed, and Joe says "now wait a minute, you know I was up to 3 am keeping that server going, and I can't be at that project meeting at $8 \mathrm{am}$, um, so most of the time there's agreement up front, but probably about 50 percent of the time the customer is actually as available as I have ask him to be.

This is another example where conflict can emerge from differences in meaning among the stakeholders for how personnel time is allocated. For this client organization, an unspoken assumption was that project work holds a second priority to important organizational tasks, especially if a production problem arises. However, for the project manager, the task availability of a customer team member was part of a firm commitment and often had important consequences because the project plan included numerous sequential task dependencies.

Along with customer cooperation, the project manager also can be faced with challenges in the capabilities and interpersonal behavior of the immediate team itself. This is consistent with how several project managers described themselves as fulfilling a quasi-supervisory or human resources role, even though their formal responsibilities were limited to administering the project tasks. In the following example of an IT project, the project manager had difficulties with a team member and had to make a judgment about keeping the specific individual on the project.

P: I had a team of about 12 engineers. They would tend to come and go based on skills that I needed at a specific time. I had a fellow come on the team who I was confident from the beginning probably had some personal issues. I didn't know what the issues were. He did tell me within 5 minutes of meeting him that his wife had just left him, so I knew there was an issue there.

According to this participant, this team member had an endless list of complaints about almost every aspect of the project and frequently disrupted the project in unpredictable ways. For example, 
P: He had made a big stink about, they were doing some work in the ceiling, and he had made a big stink about how that was affecting his breathing, asbestos was in the air, and whatever, and he went down to customer security and said "you need to get somebody up here to start vacuuming right away.”

Another problem he expressed was about salary. He apparently found this to be an argument where he could rally support for his position and cause more dissention among the team.

P: Well, this fellow, I'm starting to explain the process, and right during the middle of the meeting, he starts in with "well that's not adequate compensation, No, she's trying to screw you, don't let her do that." So I settled that down, and I you know, I said xxxx let's take this off line, let's discuss this further, but I've gone through this with management and this is the agreement.

Well, what happened is that he was able to influence another one of the engineers who was a younger engineer. They would go out drinking after work, and that sort of thing. And I got to the point where I had to pull this primary trouble maker off the project and send him home.

But I kept the younger engineer, because I thought I could manager him back into the fold. Unfortunately, it became a situation where one rotten apple will spoil the barrel and he was not amenable to behaving, he would be disruptive in meetings, he would outside of meetings, try and incite them.

In retrospect, this project manager commented that it was a mistake to not replace this employee early in the project. However, the problems she reported became worse gradually until they threatened the project's success. This lack of a clear cut event to justify his removal, along with the customer's concern in keeping a knowledgeable participant allowed the problem to grow. This example illustrates how sensemaking can be an incremental process, where additional cues unfold gradually and never rise to the level where remedial action is deemed appropriate.

Team member effectiveness problems may also be less dramatic, but still threaten the project's outcome. In the next example, the situation becomes more difficult because the team member is an employee of the client organization. Consequently, there is a reduced capability for the project manager to intercede and alter the team composition or motivate the employee to improve performance.

$\mathrm{P}$ : The hard part is when you have a user that you know is not going to be able to provide enough testing, is to communicate that to the right people. "You see over there, Suzy over there is not putting enough time into being 
able to do that." That's where it gets difficult, because Suzy is working for her boss, and let's say it's another accounting thing. And they say, well "we can't allocate Suzy more time, it's end of the month, it's quarter time, she won't have any more time, you'll have to take what you've got.” And even though you know you have an issue with that, it's the reality at times.

For these IT implementation projects that had significant customer involvement, the participants reported that customers would often commit their employees to work that would never be accomplished. A typical customer task during IT implementation would be to run test cases, in other words, fully utilize the features of the software to make sure that each function would work with a set of test data before the system “went live” on the customer's network. The most frequent conflict that arose was that the employees who were assigned these test cases would not always perform thorough checks, but report that the work had been done. The effect would be to miss problems until a later stage of integration, resulting in cost increases and schedule delays. However, because the customer was at fault, the project managers were reluctant in reporting this information completely.

Project team members were also a source of limitations because of a mismatch between their skills and what was needed for a particular task. In some cases a member with the right skill set was simply not available, so another member would be attempting to learn the skill while performing the task. In other cases, the required skills were not adequately identified because the tasks were not well defined. This occurred in the selection of an engineer who was being recruited to work on a radio design project for a small entrepreneurial organization with about 100 employees. The project manager made a personnel selection decision based on their internal assessment of the difficulty in developing the radio transmitter.

I: So the management suddenly realized there was a problem with what type of skills were needed?

P: Well, I won't say it was all a sudden but it became apparent that uh, well when I hired on as an instrument tech I told the fellow that hired me xxxx [name] and I told xxxx [name] at the time, in fact I declined the job and told him what you really need is someone with RF design experience. You know, a heavyweight with RF design experience. 
He insisted, no, no, no, you have what we want it is fine. You have the background we want. Well it turned out months later uh probably a year or more later we realized we really needed some heavy duty RF design experience on the project. And uh so part of the problem was the inability of management to really see what it would take to develop this transponder.

When the participant was hired for this position, he completed a day-long interview session consisting of detailed discussions about every aspect of the technology and his potential job functions. This example demonstrates how the significant ambiguity about new technology contributed to miscommunication about how this participant's capabilities and limitations would impact the project. In fact, the participant's repeated attempts to communicate his own limitations went unheeded. Ultimately, the failure to adequately assess this and acquire a team with those skills contributed to the problems achieving the desired goals.

Assessing needed and available resources. While team member capabilities and limitations represented undiscovered or ineffectively communicated difficulties that later become problematic in projects, the availability and qualification of other project resources were also subject to uncertainty. The ability to adequately assess these resources was another source of conflict for the project manager and other decision makers. Project managers are generally responsible for identifying their resource requirements, matching those to their available resources, and negotiating with others to fill any gaps that exist. This is frequently allocated to the project planning phase, but can occur throughout the project. According to the PMBOK (PMI, 2005a), adequate planning should clarify the required resources and assure that those resources are available throughout the project as needed. Similarly, risk plans are typically intended to serve as a backup should the planned resources not be available. However, a common theme from the participants was that utilization of formal planning tools and practices varied widely for the projects studied. In addition, most planning occurred at the beginning of projects with significantly less re-planning after important changes were made to the project. In that regard, conflict is constructed from the ongoing mismatch of allocated and needed resources, which can then become apparent in different ways. 
This is nicely illustrated in one project where the study participant was part of a consulting organization that entered the project to provide an independent review of the resources and expenditures. While this group was officially granted access to all of the project information, it was a difficult and bureaucratic effort. Nevertheless, it was clear that project resources were being expended without an adequate baseline with which to measure performance.

I: So going back in time a little. When is the first sense that things were not going right?

P: From my perspective, two days after I got here. I'm sorry, let me correct that. I came on as a project controller to the project. And one of the things I was asked to do was to look at the schedule. So, when I was reviewing, when I finally got access to the schedule, which took about 4 months. Two days later, I realized I knew that within ten minutes of looking at the schedule. I very quickly realized that we don't have baseline scope, we don't have baseline schedule, we don't have baseline costs. This thing was just a free floating ship just going along.

This project manager came into an existing project and attempted to assess the resource situation and found that the documentation deficiencies made it very difficult to make an appropriate assessment of the project. While this participant fulfilled the role of an external auditor, it was not uncommon for those who were project members to disagree about the status of needed or available resources in their projects. Many of the participants in this project were reported to express conflict by voicing a "different interpretation” of how the project would unfold or which tasks would require the most work to accomplish. In this way, the participants recognized the subjective nature of the project status as simply one interpretation of a highly ambiguous situation. Some participants who attempted to voice their alternative views were perceived as "not understanding the whole picture.” However, it was not uncommon for the participants to describe their own interpretations of their project status as incompatible with the official position. For example, on the following project there were substantial differences in estimating the state of the project and the amount of work involved for its completion.

I: OK, so the requirements document was created and signed off and the work was started, and how long before it became apparent there was big problem with the scope of the project? 
P: Ah, that would all depend upon who you talked to. I felt that it became apparent within the first six months. Um, at the time I could not convince other people that they had a major problem on their hands...I had a disagreement with the technical director and the lead design engineer because I told them they were in big trouble. And they refused to acknowledge it.

I: So what types of things did you tell them that they didn't acknowledge?

P: Ah, that they were late, how would I put it, like we did a preliminary design review, and the design was not any way near to the point where it should have been in order to do a preliminary design review. And the customer at that time who also under great pressure to get this new system in place, sort of went along with them, and let them skate through on the design review.

For technical projects, design reviews play a vital role in assessing the functionality of innovative products in early stages of development to address potential problems before they are readied for the production environment. Effective design reviews include detailed questions about all aspects of the development process to ensure a thorough design and provide a last chance to uncover potential design problems before the development process enters the next stage where it is transferred into production. The preliminary design review described in the example above is an early stage review that focuses on the higher level design and establishes the proof of concept. Recognizing that such a significant gap existed in the status of this project placed the project manager in conflict with the technical director and lead engineer who had developed a consensus about the project status. The participants indicated that this became an ongoing conflict as emerging information was interpreted differently by both parties, reinforcing their disparate positions. This dispute became significant in the project outcome because the project manger felt unwilling to begin his tasks until the existing design phase was deemed stable.

Conflicts that emerged from gaps in available and needed resources could reach beyond the immediate team and include customers and suppliers as well. In the next example, a software contractor was using a third party supplier who was totally dependent on the contract because it was the primary source of revenue for their organization. As a consequence, it caused them to 
agree to work that they could not adequately deliver. Thus, when the primary client requested changes, the project manager passed these needs on to this smaller vendor with disastrous consequences.

P: I went back to them and said we need to make changes. They made a mistake in letting me do that. It was a very small company. This was a huge contract for them, probably $80-90 \%$ of their revenue for that year. So they let me bully them into making changes after we had written a contract with them and defined what they would do. So, I was really I was really I didn't even know how much trouble I was in.

The trouble began when this vendor attempted to make changes that were beyond their capabilities, but hid the problems from this customer hoping that they could solve the problems in time. Ultimately, the vendor could not deliver on these changes, which caused the larger project to fail. However, this failure was reported to occur gradually as the project manager had to ask for increasing funding and continually delay the project in an attempt to get the needed work from this third party vendor. This incremental feature of sensemaking facilitates the decision error of increasing investments in a lost cause, when new cues which become available tend to be incorporated within the existing sensemaking framework. That is, the participant continues to value and emphasize the rational for decisions based on initial cues, even when later information invalidates them. Thus, from a sensemaking perspective, decision errors may have some basis in the ongoing integration of new information with prior beliefs.

For large and very large projects, the ability to assess needed and available resources has often become an exercise in evaluating software reports that calculate and plot resource loading as a function of time and based on a large number of assumptions entered into a project management program. Because the validity of these assumptions are constantly expected to change, the software evaluation is performed in conjunction with meeting and collaboration regarding the current project conditions. In the following example, a project auditor was hired by the client organization to provide an independent review of a major project that focused on resource loading and availability. Using traditional project management tools, this participant quickly found 
significant discrepancies in the critical path (i.e. the worst-case combination of tasks that constrain the project's completion).

I: Do you follow a process or use tools such as Microsoft project or?

P: The schedule is in Primavera, but as you probably know the schedule could be on playhouse Disney dot com [sarcastic tone]. If it's not set up correctly and if it's not managed correctly, it doesn't matter. I had one of the clients come up to me and asked me to look at the critical path. But then he asked the contractor the same question.

We came up with two different sets of answers. And I did it the way I would think any pm, scheduler would do it. The way the contractor would do it, they took all the activities that had negative flow, and then picked and choosed. They said well this one, yeah, this one yeah, this one no.

And interestingly enough, all of the tasks I came up with, and the way I came up with mine I went to the major milestones and adjusted my way back. I let the software tell me which tasks were pushing the milestone. I had about 40 tasks, not one of those tasks was on the contractor's critical path.

The major discrepancy between these two resource assessments suggests that this project is at risk because there is uncertainty in how to assign and allocate resources for future activities. This stems from ambiguity and uncertainty about the status of tasks on the timeline and which tasks are appropriately related to the critical path. Because project software tools (e.g. Microsoft Project) are the primary means of tracking resources in large projects, their appropriate utilization and interpretation becomes central in assessing resource utilization. Consequently, in projects like the one described above, the inadequate use of project tools creates significant conflict for the participants and potentially heighten the difficulties in project decision-making.

In another example of resource assessment issues, two executives were in competition for limited IT resources. These were individuals who also had other assignments to support corporate-wide computing platforms. This participant reflected on his experience with projects where one was ended because the resources had been diverted to the other project without adequately assessing the required level of support and maintenance that was required on the original project. 


\section{I: Do executives ever compete for projects?}

P: Oh yeah. Yeah they do. You have executives compete for projects and you have a person who "I want the resources to do X, Y, and Z, and another person says you can, but you're not going to roll this thing into my area, because I'm going to be using this.” And that's where you need to really, that's when you need really strong IT leadership.

Because what will happen if you don't have a strong IT leadership is you try to do both, and you have to support two competing projects, and it's a waste of cost and time. Because now short term, yes, you've made both $\mathrm{C}$ level people happy, but in the long term now you have to support both of these things, and of course you're not going to be able to get the added resources to support both of them well.

I: Are there projects that are sponsored by a weaker executive that don't get enough resources to be successful?

P: That's absolutely right. A project might be working, and then resources get pulled out to support a project by a more powerful department. And then some of those get canceled because they go on a long time without the resources needed to complete them or maintain them, and I've seen that happen on the $\mathrm{xxxx}$ project.

As this participant described, if one project wins in a competition among the executives, there is not necessarily a purposeful sacrificing of other ongoing projects. Rather, a culture of optimism combined with inadequate resource assessments frequently were said to result in too many projects being started. Several participants indicated that underestimating cost is especially problematic for the ongoing support of IT projects. In the words of one participant:

P: The excitement wanes when a project is 'up and running'. It works, people get their accolades, and then nobody wants to face maintaining that database next month. And nobody wanted to think about that cost when the project was being planned. It's ok to spend a $100 \mathrm{k}$ for a new feature, but to spend another $100 \mathrm{k}$ maintaining it? No one wants to face that, so they just don't consider maintenance. They vastly underestimate it, or they just don't even think about it.

It should be noted that these maintenance and support costs are not mysterious or unexpected by the project members. In fact the participants reported that ongoing support would be relatively easy to estimate when made part of the initial project planning. However, some organizations are structured to separate the development budget from the support budget, which can obscure the overall costs of each project. In this way, some of the unexposed conflict that is latent in projects 
can emanate from the structure of the organization, where diverse interests are insulated from collective sensemaking about their ultimately common interests.

In sum, these three areas exemplify how important unexposed differences among the stakeholders in projects may surface later to affect the projects' trajectory and, potentially, their outcomes. Such conflicts were often latent and remained undiscovered until later in the project when they emerged as recognizable problems. In some cases the participants perceived little need to communicate with their clients and customers about their needs and expectations. However, in other cases, the communication deficit was more centered on differences in meanings about the topics that were discussed. In these cases, different meanings developed primarily from individual cues as the participants attempted to resolve ambiguity about their situation somewhat independently from their counterparts. This behavior can often be modeled as individualized sensemaking (Weick, 1995), where a need for acquiring additional information and coordinating tentative understandings with relevant counterparts is perceived to be superfluous. In other words, for these participants in the projects, it makes sense to not communicate more frequently or work with others to resolve ambiguous meaning because each participant can construct meanings on their own that make sense to them. In doing so, they have an unfounded confidence in their knowledge about their projects.

\section{Unexplored Conflicts}

Not all project conflicts were seen to emerge from ambiguous communication that was driven by individualized sensemaking among the participants. In contrast to unexposed conflicts, some conflicts occurred from known differences where the participants felt unable to openly communicate and address them. This type, designated as unexplored conflicts, represents incompatible differences shaped by the influences of power in the project environment. These were projects where the participants were generally aware of a conflict but felt inhibited to openly communicate about it and adequately respond to it. For these conflicts, powerful influences either inside or outside of the project would impose restrictions on the project or its members by 
inhibiting the open discussion of their actions. These represented issues of power and voice and would be characterized by topics that were off limits or were discouraged even though the team understood that a conflict existed. In general, there were three ways that unexplored conflicts existed in the projects. They were difficulties in (a) surfacing organizational imperatives and assumptions, (b) uncovering competing interests, and (c) coping with obscured sources of power and control. Each of these is described below.

Surfacing organizational imperatives and assumptions. Because projects are typically conducted within an organizational environment, they are also influenced by forces of power that operate in the broader organization. The sponsoring organizations provide resources and constraints for their projects, and offer structural and historical processes that precede projects that establish expectations for how projects can function. In addition, the organizations themselves exist within larger cultural environments that provide norms for conducting commerce.

In this first example, the national culture of the two partnering organizations represented a highly influential source of power that affected the outcome of this project. The study participant was an American executive who was part of a project team operating in a Norwegian organization. This business contracted a second Norwegian organization (the contractor) to develop a custom integrated circuit for use in a high volume industrial application. Because the contractor was not meeting their obligations, the American executive proposed to hold them accountable by requiring them to do whatever was necessary to make the product successful by the required date. But he was operating within the social environment and cultural norms of Norwegian companies who had very different ideas regarding project performance and accountability.

I: So, were the relationships between these two Norwegian companies a factor in the project not succeeding?

P: I think definitely so. Nordic xxxx [the contractor] needed a big design win, and the carrot of having a big international company, which there aren't that 
many of in Norway, the carrot of having that on their portfolio was very attractive to them.

I: So, what happened after the problem was identified?

P: Well, I was personally in some of the meetings where I had the American attitude of "we need to crucify these guys, we need to clean their plow," because they didn't deliver. And their attitude, which to my knowledge is still there today, was more of a Scandinavian attitude of "well, we don't treat people like that. We've got to give them another chance to rectify this." But it got to the point where $\mathrm{xxxx2}$ [the contractor] was not even willing to fix all of their bugs. They would only go so far in making changes to the silicon. So, $x x x x 1$ was left having to live with some of these bugs that were permanently there.

Ultimately, this project was far less successful than planned. The product cost more, took longer, had fewer features, and contained functional defects that affected its operation. The American project manager reflected his extraordinary frustration that a supplier was not being held accountable and his organization that had adequate contractual authority to remedy the situation would not do so. In his view, this was an example of why high technology projects are more successful in the United States, where there is a "no nonsense" attitude toward meeting agreed upon customer requirements. From a sensemaking perspective, the Norwegian cultural assumptions about the value of business relationships represented strongly valenced cues that were central to their sensemaking process. These cues were not significant to the American manager who clearly prioritized the economic and contractual factors in the project.

In contrast with the Norwegian example, most participants described common practices within a specific organizational culture. For instance, a newly hired project manager in a large American organization was faced with an unwritten rule regarding the communication of bad news in her organization. This project manager, who was attempting to follow the formal project management process established by her organization for reporting cost variances, was stopped cold by her supervisor who told her:

P: We will have no schedule variances, no cost variances, do what ever you can to get rid of those variances, I don't care what you do, -- YOU get rid of them. We don't report variances in this organization -- we don't give people reasons to be worried about those costs, that is NOT what we do here. 
In this example, the project manager was new to the organization and had not learned how to appropriately shape information to meet the organization's unwritten practices. Thus, the actual rules about how to disguise cost variances were at odds with the written rules and the topic was perceived to be off limits for discussion. This specific situation may also have been exacerbated by the new hire status of the project manager. Frequently, the participants talked about perceiving their power as very restricted when they first began work because they had a limited understanding of the organization and had not yet built support networks to rely on for help. In that regard, several project managers specifically equated their effectiveness with their network of confidants, which they asserted also helped inoculate them from powerful others.

Some participants described illegal or unethical behaviors that had become institutionalized as standard practices and which would sometimes come into conflict with project objectives and the ability for the participants to perform their jobs 'correctly.' This often represented a form of reporting false information and was typically described as a "workaround" for processes that were too bureaucratic and inflexible. In several cases the participants rationalized this as part of what the organization really expected from them. That is, they believed that while they were 'officially' required to follow the rules, the culture and common practices in their organizations allowed them to bend the rules when necessary. In other cases, the organization itself was complicit in falsely reporting project activities. For example, this participant described a project which was conducted and completed without the standard documentation called for in his organization's quality process standards. This documentation was then completed after the fact by entering false information and dates.

I: So if a certain person asked you to do it, all the rules are bypassed?

P: Yea, and you try to make it look like you are following the formal procedure by backfilling paperwork.

I: Please explain that in more detail.

P: The back fill of paper work? 
I: Yea, how do you make it look like you're following the process?

P: You'll draw up a statement of work, once they implement this. You'll look at it, and you write up what it would take to implement this. You're writing up your own statement of work.

I: But you're writing it up after the fact?

P: After the fact. On a project like that you're done with the project to even realize what you need to do the project, and you backfill the paperwork. That's not the right way to do things, by no means. But like I said, politically, you have no choice.

I: So what's the purpose of having it then?

P: At one time they were talking about doing all the paperwork, follow all of the rules, you can achieve CMM level 2, that's what they were hoping to achieve.

I: What is that?

P: CMM level 2, the Capability Maturity Model. It's like ISO9000, ISO9001 certification, but for an IT shop you have procedures and you do follow-up. Really basic stuff.

I: So they were trying to get certified at CMM level 2?

P: And they never have.

I: But having that paperwork on the shelf meant that....

P: That they were following procedures.

Ironically, this organization was violating the very project procedures outlined in the CMM model, in an effort to be certified in their use of those same project procedures. It was an interesting example of collective sensemaking motivated by the sources of power in the organization and in competition with individual sensemaking that might lead individuals to reassess their willingness to violate federal law (because this was a federal contractor). Notably, this organization is a well established and well known company with several thousand employees that conducts business in a government regulated industry. The participant who reported this was had a very matter-of-fact demeanor about revealing his participation in this practice and seemed 
comfortable with the concept that actual practices are routinely at odds with the formal paperwork and espoused processes.

For a number of participants, the operation of their projects reflected larger organizational perspectives about how business should be conducted. This was particularly interesting in IT projects where two companies partnered in projects, and the companies had incompatible cultural assumptions about business. This is demonstrated in the next example, where a large enterprise software provider was performing a software implementation project with a major on-line client. This combination of a traditional conservative business culture and an entrepreneurial culture exemplified a latent conflict in their methods of operation. In this case the issue centered on testing philosophy as a key aspect of the project implementation. In response to being asked about whether she was surprised and impacted by differences in organizational culture, she responded:

P: Yeah, Right, Yes. I'm constantly surprised. I think all of us form an impression of how a successful company is run, we don't know the specifics of that but assume that they have lots of process, lots of good HR practices in place. High employee morale etc. I'm working right now I have a program of small projects that I've been managing for a, well I'll tell you who it is, it's xxxx.

Now standing outside, I always thought xxxx really had their act together, they put a lot money, A LOT OF MONEY, a lot of thought, and a lot of effort into keeping their website up, mature processes, etc. I was shocked when I arrived on site. They were putting new code into production! They had no test system, nothing! I said wow! You have got to stop this. What if you bring a server down!

That web interface to your customer is everything to you. You don't have any bricks and mortar stores. And they said, “you don't understand, we have got to get this code out there, because tomorrow morning we're going to announce xyz function on the website, and it must be in production." Well, what if you, what if you kill one of your servers, "Well that's not going to happen.”

Well, actually it did happen about 3 or 4 years ago. They had an outage for almost a day. So then I would have thought well my god, they would have torn this company apart from top to bottom, not really, they sort of slapped around some IT managers, fired a couple, brought in some hotshot fix it guys from an accounting firm or a consulting firm. There were some 
changes made. They still operate in a very seat-of-the-pants manner, which makes me very nervous about supporting them.

This provided an interesting contrast between two partnering organizations with very different cultural assumptions. The client organization consistently differed from their software vendor in their entire approach to software implementation and support. Also, they had very different approaches in how to evaluate risk and operate new software in a production environment. Such differences were incomprehensible to the project manager who attempted to plan the project from a much more conservative framework. She reported this as a consistent conflict about how to appropriately make changes in this customer's environment. That is, it was difficult to discuss and obtain consensus about the timing and accountability for implementing changes and upgrades. For her supplier organization, changes would occur more slowly and after a complete test protocol had been agreed to and conducted. However, for this major "dot-com” customer, they consistently went directly on-line with new software as soon as it was available.

Another aspect of this type of conflict is how communication can be structurally constrained within an organization, affecting both the supplier and client project teams. Some of the client organizations for IT projects were reported to have very hierarchal structures with virtually autonomous departments. The vendor project managers who were interfacing with these organizations complained about how the departmental isolation and formal chain of command would unexpectedly interfere with the project at various times. Some of these companies operated with self-proclaimed "meritocracy” which rewarded and promoted those who achieved more than their peers. Two project managers were especially critical of dealing with meritocracy-based organizations by virtue of their employees' tendency to hide and cover-up problems or even sabotage other departments for their own gain. This was reported to make their projects much more difficult to manage. In this example of an IT project in such an organization, the client representatives were actively hindered in their cooperation by another department that 
attempted to hide problems that existed in their own department. In describing this type of

organization, this participant begins with talking about how managers operate.

P: Managers... their one goal is to keep their problems invisible to their neighbor, to their peer management groups, and to upper management. And there are very different approaches to that.

I: When you say keep things invisible, you mean among themselves?

P: Yes, they tend to work very much in silos... So, this group, the system management group really has a dependency on the systems administrators and they have a dependency on the help desk because it's our software that's sending a message to the help desk, you have a server down. And then the help desk goes back to the system's administrator, says say you have a server down, or you have a disk filling up, so fix it.

There's very little interaction among these groups. Typically, it takes a minor crisis for there to be cross-pollination, discussion, communication. You don't see these guys you don't see 10 managers all sitting in a room unless the CTO has called a meeting and said you boys are all going to work together. You boys and girls are all going to work together to solve this.

The silo characteristic of this organization was reported to be even more problematic in trying to

debug a problem that occurred after the system was installed and running. The department

responsible for the problem refused to admit that they were responsible or offer the needed

information to other departments.

So we had a situation last year where there was a hardware problem. It was...something like CPU utilization was spinning up out of unexpectedly, no one could figure out why... So, it was a very painful discussion and it went all the way up to the CTO's level to get the systems admin manager to talk to the systems management manager who had identified what the issue was. Now if those relationships had existed, if there had been dialogue going back and forth, it would have been an easy thing to [fix].

Probably took us two months to troubleshoot, and we had to do an end run, kind of, we had to have a mole over in the systems admin area who slipped us a copy of the build because the systems manager didn't have an effective relationship where he could go over to the FA manager and say, "hey, can I see your build?" Couldn't get that. And we had to have a mole go in and slip us a copy of the build.

I: So, when you say slipped a copy, do you mean that he didn't want to be identified as having communicated that. Was he doing that under the table?

P: He was. He was, and it was a friend of a friend in our group who had an informal outside of the company relationship with one of the SAs [systems 
administrators], and the SA, that particular SA brought in a disk that had a copy of the gold build on it and "bingo," there was the problem.

This project manager was describing a project delay of two months that occurred because the organizational culture was one where each department protected its own territory and tactically avoided any admission that a problem was in their area. Even then, the problem was only detected and solved because of the informal communication channels provided by a crossdepartment friendship. It was an employee acting as a "mole” to surreptitiously gather and reveal information that was secreted by his department. Presumably, being identified as revealing such departmental secrets would have been problematic for the "mole" because the hierarchal structure of this organization required and rewarded the loyalty of its members to their superiors.

It was not uncommon for participants to talk about being silenced or having to work around the official modes of project communication. In some cases, when procedures were so rigid that they ostensibly forced accurate reporting, those with sufficient power could simply require their subordinates to ignore or bypass the project controls that became uncomfortable. For example, one organization (referred to as the contractor) consistently managed their project by reporting task plans that were not possible to accomplish based on the known task dependencies. They simultaneously maintained a formal project structure and timeline, yet actively bypassed the intent of the tool. It was generally known, but not openly discussed or acknowledged. In the next example, the study participant worked in a project auditing department to monitor the project activities of the contractor and was frequently frustrated with the practices and operating assumptions of the organization. She spoke at length about how the formal software tools that are designed to keep projects on-track were manipulated by the management to achieve more positive projections. She indicated that it was easy to "cheat” in M.S. Project because in this software it is possible to enter start dates that have already elapsed.

P: Primavera [a software project management program] forces you to use scheduling tools. It forces you to do scheduling the right way. In MS Project [another software management program] you can cheat a lot. You can cheat a lot in MS project. You cannot cheat in Primavera, you can’t. A 
good example, I'm often asked to review schedules. And sometimes I'm asked to review MS project schedules. Well, I'll get a MS project schedule and my first question usually is where's your today line? And they'll say it's October $6^{\text {th }}$.

Ok, and inevitably, I will find tons of at least 50 percent of the activities on that schedule that have not been completed. And they'll say, ok today is you know today is October $8^{\text {th }}$ and it'll say ok this task is going to be completed in March. But it hasn't been started yet.

What this participant was describing was that management could report an earlier estimated project completion date by removing sequential tasks dependencies [referred to later as "cutting or slashing logic”] and back-date start times for tasks that actually had not even been started yet. In her words,

P: But what our contractor does is they'll leave the milestone right where it is, and they'll just cut logic, and the task that they have to complete doesn't have to connect to the milestone anymore. So they are running past the milestone, critical work that if you don't do it, you don't meet the milestone.

I: So, they're removing the dependencies in the project time line?

P: That's just what they're doing, right, yea. And they have a procedure that they wrote that they're in full compliance with the procedure. And the client, they scream and yell and holler when I give them reports every month.

The overall project performance is often gauged by reporting deviations from a baseline schedule that would be established at the outset of a project. This schedule typically captures significant milestones, phased releases, and resource expenditures. This participant went on to describe how in her project the contract allowed for numerous opportunities for the contractor to change the baseline schedule and, consequently, misrepresent the status of the project. In her project it was common to,

take the forecast and you keep comparing it every month to that baseline. That shouldn't move. Well, in our case, the baseline moves every month. The contractor's allowed to slash logic, they're allowed to manipulate the dollars.

Because the contractor was given the authority to write the procedures, they were said to have made it easy to both stay in compliance with the procedure and misrepresent what is occurring. 
In this example, the formal project procedures called for utilizing software tools that were designed specifically to identify and prevent errors in scheduling. When using this software sequential tasks are connected to ensure that one task is not scheduled to start before the completion of a prerequisite task. So when this project consultant refers to "slashing logic," she is describing a method of falsifying the reports which are generated from the software database. By removing the connections, a required prerequisite is not identified, which makes the tasks appear to be achievable in parallel. Consequently, the project appears to be achievable in a shorter time period. She also indicated that the baseline was constantly being moved. This is akin to resetting the odometer on an automobile to give the appearance of less mileage. An accurate comparison to a baseline serves to identify project costs and elapsed times as a deviance from the original plan.

Uncovering competing interests. Another issue with power and voice is the existence of competing interests that vie for project resources or influence outcomes directly. These were conflicts of interest that represented hidden agendas or the existence of contradictory goals, which can involve organizational members, outsiders, or project members themselves. The research participants often reported that conflict emerged as they discovered, but felt inhibited in addressing competing interests in their projects. This could be competing interests in the host organization, the client organization, or outside groups. In all of these cases, competing interests were a form of unexplored conflict which tended to interfere with the successful trajectory of the project. In this example, a project manager for a large enterprise software supplier found that during the installation phase of her project, an executive inside of the client organization actively worked to delay the project and attempted to have it canceled so that his competing project could take its place in the organization.

P: I and my team arrived on site and begin installing a piece of software and within the company, someone learned, someone outside of the project learned that our project is underway and they escalate and say why are we putting in xxxx's product. 
"I have been working for weeks as you know, on yyyy's product and I think it is a better product and here's why." So my project was put on hold temporarily while these two minor executives went head to head, each trying to resell at the executive level why their product was best.

Um, ultimately, my sponsoring minor executive won out, but it did set the project back. Fortunately, we didn't have a hard deadline, like you know, the project was being announced on xyz date of the results of the project, where we had to meet that, so we had some leeway.

It was annoying, another consequence was that I lost some of my better skills, because when the project was put on hold, xxxx management came in, took my resources and put them on another project, I had to restaff, I wasn't able to restaff with the same level of skills.

In this example, the outside executive was not part of the project team and was pursuing a similar software package for a different but related application. When he discovered this competing project, he was reported to have attempted to work behind the scenes for sometime to have the competitive project canceled. Because the project manager represented a supplier, she had little influence within the organization and was mostly subject to the outcome of their internal power struggle.

However, some competing interests were also problematic for projects, but occurred at the organizational level. In the following example, the project participants were expected to essentially underbid their work to make their project look favorable at the corporate level. In this way, their project rather than an unspecified competing project in another division would receive funding. This placed the participants in a contradictory position in that they needed to voice their acceptance of and cooperation with an unworkable schedule to protect their jobs and, yet, they would later be held accountable for their performance against these problematic estimates. The first indication of a serious problem occurred during the kick-off meeting, which was organized to develop the scope of the project and get consensus from the team members. This participant first described the process of estimating the required resources and then explained how they were forced to underbid their own estimate to compete with another project.

P: When we were done [estimating the length of a potential project], the project was going to take over two years and cost close to eight million 
dollars. We were proud of our work because we were really careful not to sandbag the costs. But then, the VP came back and said "That'll never fly. If I ask for that it will never get approved.” Xxxx [another VP] will get the money for $\operatorname{xxxx}$ [name of the division], and I'm not going to let that happen. We were stunned. We looked around at each other, and just thought, so what did we do all this work for? Just so our VP can win over their VP?

I: So what happened next?

P: Well, everyone started asking questions, and so he told us, to take another hour and go back and adjust all the numbers and take half the time and half the cost out of the project.

\section{I: And what was the reason for that?}

P: He wanted this done, he wanted a really bold project that he could promote at his level and say "look what we're doing." More than anything, you could tell that he was in a competition with other VPs for who could produce more revenue and have more people under them. I'm sure he knew that there would be huge cost overruns, he wasn't that stupid. But we also knew that he was protecting our jobs.

For the participant, who was a project manager responsible for one aspect of this project, there was little point in voicing her conflict about unreasonable task deadlines. In fact, the original kick-off meeting so effectively set the tone for the project that, as she indicated, little discussion ever emerged about the "elephant standing in the middle of the room.” That is, seldom did the team openly address the conflict between their unrealistic project schedule and the actual work that was being accomplished. She indicated that her team would work on shortcuts or try to find ways to speed the project up, but there was a sense that the deadlines were not really real so they went by without too much consternation. Ultimately, this project failed as it consumed its allocated resources long before accomplishing a functioning prototype. However, by this time, the VP had moved on to another position, and was not held accountable for the failure.

In some cases project team members are both the producers and consumers of the project, which adds additional complexity to negotiating resources and requirements. This was especially true for an institutional organization with several large branch institutions, each with their own project resources. In a project to upgrade technology and provide enterprise software, the larger 
branches felt their needs were more immediate than the other branches and worked secretly

together to split the project into two components.

P: It became a political issue that you've got 3 out of 8 [institutions] who have the resources to proceed and the rest that don't. What are you going to do? The 3 were pretty vocal that they weren't willing to give up.

I: These were team members?

P: Yea, because you need a representative from each institution. And that they were prepared to escalate it up the chain of command. That they had no hesitation of moving it up. And they did.

I: So did these three people get together and meet among themselves?

P: Yes, the formed a little troika, they went off on their own and plotted.

I: OK, so this was before you knew about it?

P: Yea, so they presented a united front to me as PM, and said "ok, this is what's going to happen, because we feel strongly enough about this project that we're not going to let it go.”

Because these individual institutions had responsibility for the operation of their own divisions and exercised control over their respective employees, they had sufficient power to overcome the will of the other members. The broader organization supported this because their emphasis was directed toward maintaining a reputation for "excellence” at one or two of their premier institutions at the expense of the overall system, which included a number of satellite institutions. This was reported to produce an atmosphere where the three predominant institutions felt entitled to advance their own agendas over the needs of the other member institutions. They took control over the project during a meeting where they introduced their own plan.

I: So, what happened during this meeting...

P: They had a handout which was in black and white dollars and just said, you know, we've gone through the budget, we've taken a look at who's got what resources, and we've got them and we're unwilling to wait for the rest of the group. And we're going to split this project off, and do it on our own.

I: So without those resources, the rest of the team has trouble making progress?

P: They're dead in the water. 
I: They're dead in the water. But did they have some authority to do that?

P: Well, they went and got it. They went right over my head and went to the president's of their institutions and explained the situation and ask permission to break off.

I: So, they were working behind the scenes?

P: Macevallian even.

This project was somewhat unusual in that the project personnel as well as the project budget were apportioned to each executive who had complete control over that portion of the project. Further, because two of the institutions were considerably larger than the others, their management had more power to influence the project activities. Essentially, this asymmetry of the power allowed two of the more powerful members to dictate the direction of the project and out-maneuver the project manager. Interestingly, the relationships between the two groups stayed intact during this change and at the time of the interview the outcome had still not been determined. Here, sensemaking occurred at both the individual and collective levels to create distinctively separate interests among the stakeholders. Notably, the formation of a sub-group among two of the largest organizations is reflective of how the broader project with its slower pace did not make sense to the sub-group. However, even though it resulted in breaking off and forming a second project, the organizational norms still provided a cohesive nature to the group, which facilitated the ongoing cooperation as the project proceeded.

A final example of how conflict can emerge and become problematic from competing interests is related to differences among team members themselves. An often unchallenged assumption about project teams is that their members are often aligned with the goals of the team and subject to the authority of the project manager. However, for some projects, the project manager has limited authority over his/her team members and conflict can emerge and become especially problematic when the team members have competing interests regarding their work products. Such was the case in this example of a project manager who was careful not to alienate 
her team members even though they were devoting substantial time to "gold plating” their work at the expense of completing it on time.

The practice of "gold plating” was described as occurring when team members add in additional features that are outside the scope of the project. Frequently, these are software “extras” that may be totally hidden from the organization and created as a way that team members can personalize their work or show off to one another. When asked about gold plating, this project manager replied:

P: It's absolutely forbidden, it is. When we do the charter and scope setting that is one thing that is absolutely forbidden here.

I: Have you ever seen it happen?

$\mathrm{P}$ : Oh, sure people try to do it all the time, because they want to impress somebody. "Look how smart I am."

I: So there is cost or time impact for these things?

P: Of course, there always is but I have been unable to find a way to stop them. They are Easter-eggers here.

I: Which means what?

P: Easter eggs are the special features of video games that are hidden. And you do a magic key combination or you on one screen click on the right corner and you get an Easter egg. Well these guys all like video games, and they put Easter eggs everywhere.

While this project manager is responsible for the software development projects in her organization, she perceives little control over activities such as these. Because the programmers know that their jobs are secure and cannot be impacted by her assessments, they were reported to almost flaunt their ability to add these "Easter eggs" at will. She explained further why she held little power in controlling her team.

P: You see that's another thing that's different between private industry and, I worked in both private and public sectors. In the public sector they can't get rid of you. You've probably heard of that before. And there's nothing that a PM can do to a team member as far as performance reviews that means anything. 
I can protest it till the cows come home, it means nothing. Again, you've got to know which of your battles to fight. And all you'll do be really having a fit about it and putting something in their file or asking to have something put in their file, is you'll piss them off and they'll never be a good team member again, so I don’t pursue this.

And gold plating is an absolute no-no, but they all do it, and they do it all the time, and there's nothing I can do about it, and I don't want to alienate the team.

This situation was particularly interesting in that traditional assumptions of power residing in the project manager were effectively reversed. The project manager admitted that part of being successful in her job was to avoid alienating the project team. In that regard, the projects were always conducted with the backdrop of somewhat competing interests between the project manager and the team members.

I: So if you have the situation where you did alienate someone you might have to have them as a team member again?

P: I know I will.

I: What might they do if that were the case?

P: Oh, they may be an obstructionist or actually a saboteur. Your worst nightmare and you probably hear this from other PMs, is to have a saboteur on your team. That's someone who actively works against your interests. And if you really piss somebody off they'll become a saboteur. They'll make you fail, and hold you up for ridicule and then your credibility goes right down the toilet. So you would never do anything to turn someone into a saboteur.

This project manager explained how she continues to be influenced by a project that occurred several years ago, where one of her team members became a saboteur and secretly worked to make the project fail. She explained that he was motivated by being denied a promotion and acted in retaliation. As a result, she revealed that a significant amount of her time is allocated to ensuring the satisfaction of her team members, which would other wise be used for solving project problems.

In sum, competing interests were influences on the project from both internal and external sources of power that became problematic with regard to project outcomes. In some 
cases these became visible as executives competed for their own projects. However, as shown by the last example, sources of power were also found in lower levels of the organization and among the team members themselves. For these conflicts, power was diffuse and negotiated among the participants in the form of cohesive goals. However, the issues of potential interferences and sabotage were never discussed. Overall, these were competing interests that constrained the participant's communication and ability to act in accordance with optimizing project outcomes.

Coping with veiled sources of power and control. A third form of unexplored conflict was represented by veiled sources of power and control, which were reported to originate from diverse sources and have distinct influences on projects. In addition to sources of power that emanated from organizational imperatives or competing interests among organizational participants, there were other examples of power operating to enable or constrain certain project activities over others. These were often more subtle sources of power and from unexpected sources. For example, in this case several project members on the team were about to retire, which made them both powerful and powerless as they contemplated their role in correcting a major problem. This project member acted as an informant, reflecting the position of other members of her team.

It's just, the environment is not an environment that people are happy with. There's a lot of people close to retirement, so a lot of people are just saying "I'm just going to ride this boat out and I'm going to retire in a couple of years, and you know so be it.” And there's a lot of just giving up on trying to scream and yell, the emperor has no clothes. Just let him have no clothes.

When this participant used the phrase "the emperor has no clothes" it was to indicate that while power operated to constrain their voices, the project participants could easily see what was occurring. By saying “just let him have no clothes,” she expressed the opinion that these participants had given up on trying to correct the problems in the project that would make it successful, and just resigned themselves to earning a paycheck until their upcoming retirement. In this way, while power operated to silence them, their dependency on the organization had virtually ended, which made them relatively passive participants. Essentially, these employees 
had power because they were at the end of their careers and had little to lose by being controversial, but they also had little to gain. This participant reported that it was more important for these team members to enjoy a peaceful environment than to rock the boat and improve a system that they would not be a part of for too much longer. Therefore, they were reported to be more likely "to look the other way" and accept unethical behavior by those managing the project.

In the continuing discussion about the needs of the employees and their response to the project, I asked more precisely how their employment status may have resulted in altered behavior among team members. For those who were not near retirement, power operated very differently. This participant suggested that those participants were often intimidated by the project management:

P: In some cases they will compromise their own values and integrity. Because you know, “if I speak up I'm going to lose my job.” You've got to make a value judgment there. We've got some cases where you've got single family, single income families where the man or the woman is working and that's the only income there...

For this participant, the single income situation did not apply, and she felt more powerful in standing up to unethical behaviors that were occurring in this project. However, she recounted how two of her team members were so concerned about losing their jobs that they agreed to falsely report their progress to satisfy their project manager, which made his report appear that the project was on schedule when it was actually far behind the stated timeline. From a sensemaking perspective, the participants in this project constructed the rationale that while projects might be behind schedule at one point in time, they have numerous options to catch up and, therefore, the falsification of data is only a temporary action.

This next example is of a very large project where oversight was provided by a local office of the sponsoring organization. This group was designated as the M \& O [management and oversight] office and had primary responsibility for ensuring that the contractor fulfilled their project obligations for the parent sponsor. The participant I interviewed occupied a management position in the consulting group and was contracted to provide an independent assessment of the 
project. The project management consulting organization was tasked with monitoring the project.

However, they were unable to stop the local sponsor from paying for the large cost of a defective design.

P: The M \& Os contract says give us a design that will work. So, in my mind, I think, well if their directive is, give me a design that will work, and this design has this one critical flaw in it that the design won't work, why give them more money?

And that's exactly what they did, they went to the client and said give us more money so we can fix this critical flaw. And as soon as I read that, my first comment was why are we giving them money to solve a problem that they [the contractor] should solve because the design is their problem not ours? The client approved it and gave them the money.

This project auditor attempted to intervene and keep the contractor from charging for extra work that should not be absorbed by the client. However, as an independent third party auditor, she described herself as quickly getting in over her head, into the politics of the project. The auditing firm was earning significant income by reporting that the overall project was being effectively conducted. This was an important conclusion for both the contractor and the customer representatives who were both held accountable for the overall progress. However, because there were substantial problems, this information was constrained and redacted from reports. This was most apparent in reports that covered major project deliverables.

P: Right. Exactly, I'm a full believer that the client has to have a certain amount of privileged information and there are going to be certain things that you cannot divulge to your contractors and your staff. But, this is things like major major milestones, MAJOR deliverables that we cannot talk about. No one can talk about when it's due.

For example if it's written in a report, it's removed. If it's, if we're in a meeting, they'll just stop the discussion. And we're all going, but wait a minute, this is a major, major deliverable, and we're not allowed to talk about what it takes to get this thing done. We're not allowed to talk about the date that it's going to be due, or how long it's going to take.

As she indicated, one of the consequences of inhibiting this type of basic project information is that middle level managers are often mystified by the decisions that are issued from higher levels of the project management organization. Consequently, they are unable to make logical choices 
in managing their own sub-projects and make decisions that coordinate with other members. In this way, contradictory information and a lack of communication about the underlying rationale for project decisions is consistent with stunted sensemaking (Weick, 2001). In effect, the participants give up on the search for consistency of direction and resort to more basic overarching explanations for the project behaviors that they observe among their leaders.

Another source of power that is not always obvious to the project stakeholders is the ability to control the content and timing of project gating functions. These are major milestones, meetings, or project deliverables that must be completed before moving to the next stage.

Frequently, these approval points are controlled by individuals outside of the project itself, but who can exert a strong influence on the project by their ability to define the successful completion of these milestones. This is apparent in the following project where the project manager actively manipulated project reviews so as to avoid the public recognition that the project was severely behind schedule.

P: We were supposed to be a catch up program to catch up to the civilian one at critical design review. And the civilian program would never admit to the size of the problem that they had. So whenever they would announce a delay, it would always be six months, things were going to be delayed six months.

I: And did they know that it was more than six months?

P: I'm sure there were people there who knew it was more than six months, yeah. They should have announced they were at least two years from completion.

I: And what would have happened if they had said two years instead of six months?

P: From our perspective on the military side, we would have shut things down until they got to the critical design review point that they were supposed to be at when we started our program.

The participant was completely convinced that the practice of estimating six months was a tactic to keep the project alive. However, the program manager had the power to issue those estimates 
and keep the project alive. The consequences of this were significant for the military team because,

P: What we ended up doing because they kept announcing 6 month delays, we kept planning our program over and over again. Where if they came out and admitted a two year delay, we would have just put everything on hold, left it, and came back and started it two years later.

I: So did you believe them when they said 6 months? Or you just had to go along with it because that was the official message?

P: I had to go along it because that was the official line.

I: Sounds like it that you didn't really believe it though.....

P: No, I really didn’t believe it at all. No.

In this project, the civilian project manager had the power to make sense for both project teams by establishing milestone dates. While these could be questioned in private, the project participants lacked the power to officially question the viability of new dates in any meaningful venue. Therefore, each time a new date was announced that did not make sense to the participants, they were expected to work toward the new date, even when they perceived it to be completely unrealistic. By asserting the importance of a "positive attitude" the management had the power to frame alternative estimates as being disloyal and unproductive to the team.

In this next project, a small organization included a number of employees who were also owners as well as engineers. This organization had one major engineering project which was behind schedule and becoming an economic burden for the company. As this project continued and became more costly, there was considerable dissention between the president and the remaining participant/owners. While this was occurring outside of the development project, the president was under increasing pressure to make the project successful or to show leadership in making a change. The project manager had some awareness of this as revealed in the following comments.

P: There was a lot of dissatisfaction with the way [that the president was managing the company], in fact I was very dissatisfied with the way the president was managing things and of course, my voice didn’t count at all. I 
was very minor, very minority stockholder, but the VP's and others who owned quite a bit of the company and they got together and fired the president that afternoon.

I: So was he one of the owners too?

P: Yeah, $\operatorname{xxxx}$ [name of president] was one of the founders of the company. He and $\mathrm{xxxx}$ and $\mathrm{xxxx}$ were the founders.

I: So there was a group of owners who were working there and .....

P: yeah, see that in reality was the major problem, the owners were working at the company, so they felt like, being owners, well they could dictate policy and projects and, and you know, control they had the right to control the project and to decide what the product would be. They could say let's do it. And they did. And the result is that is a very inefficient engineering project.

Prior to this sudden change, this participant had worked in this company for several years. The group of owners was reasonably silent and fairly innocuous as the company grew rapidly. The tide began to turn when changing technology made their primary product obsolete. As cash flow decreased, this was the catalyst for the additional scrutiny on the overall direction of the growing cost of the particular project that this participant was managing. Relatively quickly, the previously silent owners were exercising their considerable power and taking an active role in reorganizing the company. This was a surprise to the participant of this study who had assumed that the other owners were in concert with the president and were in support of his management.

Overall, unexplored conflicts represented issues with power and voice where projects were affected by the difficulty for participants to make sense of the forces that were influencing project activities and outcomes. As demonstrated by the analysis, these influences came from a variety of sources. In some cases, organizational norms and structural artifacts created a silencing effect that constrained what communication was appropriate for the team members, limiting their effectiveness at solving project problems. In other cases, there were conflicts of interest, which may have only been partially apparent to the participants, yet, were implicated in project outcomes. Finally, project members also found themselves coping with hidden or unexpected sources of power that were shown to exist at several levels. In all, power operated to both enable 
certain behaviors and constrain others, including the perceived ability for the participants to communicate about their differences and find solutions to their project problems.

In these examples, sensemaking was both an individual and a collective process as the participants attempted to cope with the influence of unexpected sources of power in their projects. Because power often worked to silence individuals and constrain the information that was available for understanding, individual sensemaking allowed participants to construct meaning from their own cues, resulting in relatively autonomous decisions and actions. That is, the participants indicated that they often relied on their own prior experiences to 'make sense' of what was going on and know how to respond. However, sensemaking also operated collectively as individuals shared information with each other in an attempt to understand why unexpected decisions were made at the organizational level about their projects. The participants often described how "the word got around" or how the "oldtimers" knew where the "real power" was located. In this way, the project managers would report having an informant that would help them understand what was influencing their projects.

In some projects, open conflict existed but its expression and endurance was not perceived to be dominated by issues of power. Therefore, by conceptually distinguishing the role of power from other project effects, a third, distinct category of conflict was found among the study participants. That is, unresolved conflicts were a consistent theme of participants who described a number of reoccurring disputes that interfered with progress on their projects.

\section{Unresolved Conflicts}

Unresolved conflicts were those that were often widely known among the team and represented ongoing differences about how the project should continue. These are described as issues with action and timing, because they tend to center on the theme of how the failure to resolve important issues within a specified time frame may cause opportunities to be lost. Timing was particularly important because "windows of opportunity" would close at different points in projects which would reflect lost opportunities if conflicts were left unabated. Primarily, 
unresolved conflicts were represented by three categories. These were difficulties (a) adapting to change, (b) agreeing on priorities and scope, and (3) negotiating solutions for problems. Each of these is described below.

Adapting to change. While projects sometimes fail because of missed requirements or other limitations, a critical opportunity to make projects successful was to adapt to new information and conditions in a timely and comprehensive way. In the words of a senior project manager with over 22 years of experience in high technology projects:

P: Well, no matter what anyone says about project management, it's about finding balance and adapting to change. You have to balance your ability to solve problems that come up, deal with the evolving and changing requirements, the business pressures to make money, and the different interpretations that everybody on a project has. That's why you can't really have an overall criteria for success. Because all of those factors are different on every project. And you're constantly dealing with something new, some surprise because no one can account for all the contingencies that might happen.

This project manager described his primary role as both the architect and facilitator of change.

Along with other participants, he recounted examples where team members who were previously in agreement, developed different understandings for the nature and impact of a particular change that emerged in a project and then had difficulty in achieving a post-change consensus about the appropriate response. This was said to be particularly problematic when the team members were only partially convinced that this change was necessary or led to a more successful outcome. From a sensemaking perspective, difficulties with adapting to change represent the condition where collective sensemaking had been previously achieved, but lost when new discordant cues become salient to the project. Under these conditions, individuals differ in their assessment of the new information and have a limited time to reach consensus.

For some of the high technology projects in this study, adapting to change was grounded in technical uncertainties which unexpectedly became problematic during the project. These were issues that had not been adequately addressed by risk management, so there was not a 
readily available solution. The following example illustrates how a carefully planned feature failed to work, requiring significant technical change for the team to adapt to.

P: I was working on a project and there was a security feature. And we thought it was corrected and we went there, and we hired a professional hacker, basically, to hack into the system. And the security feature that was supposedly fixed, wasn't. Well, it was, but the guy was still able to get past it. And that came at the end of the project, which was bad, because it's what do we do with security features that are there but are not working?

I: So, in this case, what happened?

P: We worked at developing a contingency. We couldn't use it as it was originally designed, so we looked at things we could do with other pieces of equipment to enhance the security.

I: Does that mean you worked around it?

P: Well, ahh, sort of. Instead of having security at the device, let's say security up there [motions with hands, above head], we went to the next layer down. So in this case, it was a device that plugged into the network. And so instead of having the device have the security on it, the security went down to the switch layer, the Cisco layer. And said hey, ok, this is what the security is now.

I: So, was it the complexity of the system or what?

P: Part of it was the complexity of it, part of it is, we're not professional hackers, so we didn't have the expertise to go in there. I mean a lot of things that we stopped ahead of time, but there was things that he was able to do that we didn't know about, and now we do.

The team was able to agree on this change because it appeared to provide most of the intended functionality and there was no other plausible alternative. This story was rather typical for descriptions of IT projects where a defect in the functionality was found late in the project. The participants attributed their success to the team's willingness to give up on their original approach and "go back to the drawing board" to consider other designs. This ability to adapt to problems was less apparent in other reports where participants indicated that their teams were reluctant to "go backwards” on projects once a particular phase had been completed. This reluctance is consistent with the culture of project management, which places a significant emphasis on phased releases, and holds executive sponsors accountable for their interim approvals. From this 
perspective, sensemaking includes valuing the cues that are consistent with following prescribed project processes.

Project teams also were challenged with adapting to unexpected organizational changes that threatened their projects. For example, in one particular international project, a sizable number of team members were working in $\mathrm{xxxx}$ [country] on temporary visas. In the normal scope of the project, visa expiration was simply not an issue. However, as numerous problems mounted, project delays caused several employees to reach the end of their work visa periods. Because, this was not perceived to be a significant problem early in the project, there was little risk assessment for this contingency.

P: At the end of 5 years, they had to leave or take out landed immigrant status in $\mathrm{xxxx}$ which means you say that you are planning to stay there permanently. So we had a big turnover of people when we hit the 5 year mark.

I: And did people take any kind of actions to try to head that problem off.

P: Well, we did hire a lot of people locally. With the original intent that they would be able to take over and run the whole show. That worked to a certain degree. But not some key positions. They also brought some other people from the states... It was definitely a knowledge expertise issue. We did not have the depth of experience in $\mathrm{xxxx}$ [omitted for confidentiality] that it turned out that we needed to have.

I: So the fact that all these people had to leave at the 5 year point and it was shifted to local people um, what did that result in? I am trying to understand the impact of that.

P: Yeah it did cause delay. There were a whole bunch of things that had to be added that it did affect. There was training and new skills that had to be learned and it was a constant effort to get new people up to speed.

I: OK, and how many people on the team did that affect?

P: We had uh, at our peak staffing, we had about 350 people there of which about 80 were people sent from the US.

During the last year of this project, the problem of expiring visas increasingly became the primary topic of conversation in project status meetings. However, since this problem was not perceived to be a risk previously, there was no planned course of action that made sense to the participants. 
Ultimately, the inability to both recognize (unexposed conflict) and then adapt to (unresolved conflict) the situation led to a sudden turnover of employees with virtually no time to transfer knowledge and training the new team members. In this case, collective sensemaking failed to provide the group with a consensus for taking action early enough to accomplish a smooth transfer to the new team. In this organization a sense of optimism alone with an elevated sense of influence was reported to pervade the meetings. The participant reported that when the problem finally seemed inevitable, much of the discussion focused on finding new ideas and 'out of the box' solutions. As time went on, the hope for a magical solution dominated the conversation at the expense of the more pragmatic plans that were ultimately implemented.

Timing was an important theme when the participants were describing their teams’ acceptance of and adaptation to problematic situations. Because technology was often described as rapidly changing, the participants felt that windows of opportunity would open and close during the projects that offered them a limited chance to adapt to these changing conditions. However, this required a timely acknowledgement of the problem followed by a consensus for solving it. In the following example, a product that was in the design cycle failed to receive market support during early prototype demonstrations. The management's overly optimistic assessment prevented the team from identifying weaknesses and adapting the project until it was too late to save the product.

P: They just wanted it done, and they didn't really understand too much about the technical features or the competition. They were financial types, which was fine, but I had the sense that they were just turning a blind eye to the fact that this thing wasn't going to go anywhere.

I: What gave you that sense?

P: Well, that it was clear that technology was changing, new products were coming online that had better performance, but what we were hearing coming from them started to sound like everything's going great. -- We've got this new partner, we're going to this trade show over here, and the potential is unlimited, but it was what they didn't say that came through really loud.

I: And what was that? 
P: Well, they never talked about any real sales. We would ship out beta units for places but nobody really sold anything. It was always the cheerleader talk about what was GOING to happen.

I: So as the months went on, was there any sense that it was time to consider any changes to the project?

P: Yeah, there was a window of time for design changes, but after that closed, it was like, you've been dealt your hand, and you have to play it out.

I: So, during that window...

P: During that window, if we knew what changes we needed to make, we could have incorporated them into the product, and had something very different.

During the last several months of this project, the team members (which excluded management and marketing) were reported to have raised numerous 'red flags' about how the features of their new product were falling behind newly released competitive products. However, the management position was that adding features that would delay the product release and that was potentially more problematic than marketing the original version. Importantly, the team members were not powerless to make this issue more visible, but according to the participant, the window closed very gradually while the organization increasingly relied on a heroic solution. Thus, this project failure reflects how unresolved conflict between the project sponsor and the team can exist as disagreements regarding change and how to adapt to it. It differs from the other forms of conflict primarily because changing conditions introduce new sets of cues to be evaluated late in the project, which may be inconsistent with the initial assumptions justifying its funding.

As shown in the previous example, changing environmental conditions (e.g. competitive pressures and new technology) can create a need for adaptation. However, in the following example, internal business changes at the executive level caused the ongoing project design to be incompatible with the new vision of management. In this example,

P: The project itself, went fine. It did what it was meant to do. OK? And the project was on time and in budget. I mean everything worked out fine. The team people were very good, and the end product was actually very good too. But what was happening that we did not know about was that they were at the executive level deciding that they wanted to make an ultimate business change in the way 
they did their compensation which affected our system. That is what we were collecting data for. Um, so...

I: So your system couldn't be adapted to do that.

P: It could have been. But then they would of had to put more money into it. And they decided not to. So... we gave them an estimate of how much time it would take for us to re-do what we had done to fit their business needs, and they had someone in place who said no, we just want to kill this software. So, it is just very interesting the way it all happened, and after two years of development, they scrapped it all. But that happens, it does, it truly does happen.

In this example, the internal project lasted almost 24 months and then was completed, implemented, and was utilized on the organization's IT enterprise infrastructure for approximately 6 months before it was shutdown. According to the study participant, the major gap between its functionality and the new corporate vision could have been avoided if the executives would have communicated their vision earlier in the project (unexplored conflict). Nevertheless, the plans were known far in advance, but kept secret from the IT team to avoid the potential for leaked information to have a detrimental business impact. In addition, the decision maker was reported to have a preference for vendor supplied software, which represented a competing project for this team. Therefore, the project itself suffered an ill fate, not just because the information was unavailable or because of competing interests, but because the team could not adapt quickly enough to the changing business environment that they were immersed in. Here, the conflict about the competing product was conceptually separate but practically related to the conflict caused by adapting to the new vision. Had some of the information been available earlier, the software could have been made more flexible, resulting in a longer life for the project.

Overall, the participants were consistent in their reports that ongoing projects often encountered significant changes that created conflict about how their teams should respond. One participant described how this is frequently a critical project issue.

P: Yeah, yes, change of actual software in the middle of the project is very important. You get into user acceptance testing, and you know, you have the user looking at this and before you've got them involved and hopefully you are getting their buy-off and you are showing screens and showing them process flows. But then when they finally get their hands on the system, they go, you 
know what, we really need this. Or you know what, we need this extra report or we need this extra set of screens or we really have this functionality. And that it is something that is so important that you manage correctly. Well, ok, either try to deal with it or you know, ok go ahead with what we have and we will do a phased approach, or you judge: would you use this system at all if you didn't have XYZ functions? And if they say NO, we can't use it without it, then you have to try to figure out how to mitigate that or decrease the risk of it, or how did you miss that kind of thing? So, change within itself, within a project, is I think one of the hardest things to manage. But if you can't adapt to changing requirements, then most of the time, your project's dead.

The ability for project stakeholders to acknowledge an important change and act within an appropriate time frame may reflect their success at collective sensemaking. That is, these teams who adapted successfully described how they gained consensus for what the essential issue was and how best to proceed. In contrast, for other projects some important opportunities went unused when the participants continued to rely on separate data and failed to collectively acknowledge how the changing environment altered their basic project assumptions.

In sum, there were a number of projects in the current study that were at risk or failure because of unresolved conflicts with adapting to change. These conflicts were generally known and discussed among the members, but could not be resolved within the window of opportunity to act with efficacy. In other projects, a related issue became a source of unresolved conflict for the participants. That is, the stakeholders at times struggled to obtain consensus on the priorities and scope for their projects.

Agreeing on priorities and scope. Traditionally, scope is seen as problematic in terms of its growth throughout a project. The terms "scope creep” is commonly used to describe small incremental additions to features or functionality which are incorporated without utilizing an official project change process. In this way projects are at risk because there is not a corresponding increase in resources to match the added requirements. The current discussion on scope and priorities incorporates the concern for how scope changes impact project success, but does so by focusing on difficulties that the project stakeholders have in identifying and agreeing on the appropriate scope for their projects. Because change is a frequent aspect of high 
technology projects, several participants reported that it was easy to lose track of why a project was being conducted, how it would be used, and exactly what features were really needed.

Because of that, one project manager was very insistent that a statement of work was incomplete without a "vision" for the project.

P: I think getting back to the broader goal or overall vision, that's where a lot of projects break down. We've found that if you don't clearly articulate what the vision is, people won't follow you. They'll get half way through the project and then say, what are we doing that for again? You've really got to constantly focus on this is why we are doing it. When you focus down here [motions lower] then we won't accomplish it.

I: Is the requirements document enough for that or are there other ways needed to communicate vision?

$\mathrm{P}$ : In the beginning you have to set the tone and say what the project is and why you are doing this, probably something that follows the business case. Here's what we're trying to accomplish. There has to be a need somewhere that the team agrees to. We're filling that need, this is the vision of what we are trying to accomplish.

This participant clearly articulated how a vision could serve to communicate the intent of a project and, thus, fills in the gaps for requirements that are not clearly defined. In this way a clearly communicated vision establishes the scope for a project and guides the participants in establishing their tasks and priorities. However, most participants indicated that their projects were not associated with an over-arching vision statement. Rather, the team members typically inferred the vision from the project leader presentations during an initial project kick-off meeting. Consequently, conflict over scope and priorities became problematic in a number of situations.

For example, this project manager described a failed project that was attributed to an inability to agree on a scope.

P: We had a project that we actually brought in a consultant with and we tried to tell them what we needed. And we had a big one, but nobody even had a clue of what the scope was. The worst thing that could happen to a project.

You don't have a clear vision, you don't have clear objectives and goals and you haven't fully defined the requirements as you said. How can you define the scope of what you want to accomplish? And this project went from "we think we need this" as the project went on, then "we think we need this too.” 
And every time they opened the door to one, a whole new set of requirements came out of it. A whole new scope came out of it.

And this particular project, I say it failed miserably. The proof of the pudding is, we went probably 3 to 5 years to get this project up and running, and they finally closed it down. The vendor that we chose to help us with this, they don't have a clue.

So we went out there and said "we're going to take this direction." We simply fired them, we went this direction, and we begun talking to a number of vendors. We said we need this type of system that can do these types of things.

We actually ended up landing on two vendors. We combined their resources to come up with the product that we wanted. Meanwhile, this other vendor was left scratching their heads, saying "well we did everything you asked us to do." What it really came down to, was there was serious lack of direction for exactly what it was that we wanted to accomplish. We thought we had a vision, but it was such a loose vision that it was never embellished with the details. We never communicated it to our vendor.

This was a multi-million dollar, multi-year IT project in a medium-sized organization. While they held monthly project meetings and submitted to quarterly management reviews, they were continually unsuccessful in establishing a scope to guide their activities and decisions. This participant reported that the team repeatedly failed to agree on the primary end user for the product, specifically how it would be used, and what future application would be. According to this participant, "we tried to focus on everything, and if you focus on everything, you focus on nothing.” Further, he expressed that their primary failure was in clearly defining lower level tasks without connecting them together with an overall vision.

Project priority was another form of unresolved conflict that was attributed to project outcome. The participants described how their projects may be shifted by the organization from a high to a low priority for any number of reasons. The change may be reflective of changing organizational priorities, a new sponsor, or a program manager who loses confidence in its potential to be successful. In the following example, a participant was referring to a specific project that was seen by the division vice president as a "flop in the making," so the team spent 
more and more time trying to obtain sufficient resources to keep it alive and prove that it could be successful.

P: So, the project dies a slow death because it's put on the low priority list. Then it can be pushed aside and quietly killed without anyone looking like they failed. Sometimes a lot of extra costs are involved because no one will just come out and say "this project has failed, so let's kill it." No one wants to be associated with a project that is labeled a failure, so they just barely keep it breathing for months until it dies a slow death.

I: And that is justified because it is labeled as a low priority?

P: Yeah. Making it a quote "low priority" is the way to hide a project failure. Or sometimes, to just make it go away, if the company loses interest in it. There is always something new and more exciting that is being talked about, like "if we just had the resources, we could do X Y Z, -- where can we get resources for that?"

This participant recalled that his project was ongoing for several more months while the team tried to make it successful as they battled for a higher priority status. Eventually, they lost enthusiasm as it became more apparent that it would be terminated. However, this idea that priorities and scope were both fluid in projects was a common theme for many participants. Even if the statement of work was well written and unambiguous, it was common for stakeholders to request new features or re-prioritize activities. In some cases, team members reported that they would attempt to intervene to make their own projects a higher priority and ensure their continued role on their project teams. This was a form of purposeful sensemaking for the participants who searched for cues to create a rationale that would keep their projects alive and vibrant.

In other cases, customers would attempt to add requirements during the course of a project in an attempt to receive additional value without additional cost. The following example is one project manager describing how she dealt with customers and clients who often attempted to add requirements during a project.

P: Typically, a customer will approach me in a meeting and say "I forgot... the purchasing group needs to have a copy of this form bla, bla, bla. I know your software can fill that form in automatically."

I: How do you respond to that? 
P: I tell the customer that we certainly can change, add, delete requirements, uh, the way we do that will be through a formal change process and what I do is prepare a an estimate of an impact estimate if you will. What it will do to the schedule what it will do to the cost what it will do to quality if it impacts that.

Uhm we then take, I then sit down with the customer and they agree or disagree, "I can absorb the impact of my requirement change." Typically [laughs] they unless and I can't remember a situation where uh, a customer agreed to absorb a very expensive change.

Typically that's the point at which they impact how important a requirement is. And typically they'll back off, or another very good technique is I can, I'll say why don't we put this in phase 2. Why don't we recognize this as a needed requirement, but not in this phase. And they tend to like that, whether phase 2 happens or not.

This project manager who manages IT implementation projects routinely negotiates the priorities and scope of the project with her customers. She acknowledged that while many changes are substantial enough to require an official change to scope, the smaller changes were made with informal agreements (even though that was strictly prohibited in her organization) between the parties. She indicated that "the danger of doing that is scope creep - the little things soon add up, and now you've got a bigger project.” However, along with other participants who managed this type of project, she asserted that it was important to accept some changes to build better relationships with customers and, as a consequence, be perceived as "reasonable" or "flexible" rather than "hard-nosed" or "legalistic.” This flexibility could then be used by the project manager (i.e. via social exchange) to ask that some problematic features be postponed until the next phase. In this way, these informal negotiations that represented "off-the-table" agreements were deemed to be important tools to be successful in spite of the formal processes that were in place.

Some of the participants who worked on projects in smaller organizations revealed the difficulty in gaining consensus about how projects were prioritized and how their tasks were structured. Also, the informal environment of smaller organizations often lent itself to significant ambiguity about the scope and activities of projects. In the next example, the context was a small entrepreneurial organization of about 100 employees where several owners were also engineers 
who participated in new product development. The project described in the following section

represented a major new product initiative that would incorporate an important emerging

technology. It was to become the central product for this organization and one that would replace

their existing technology which was their primary product for several years.

P: We really had very little in writing in terms of performance requirements or goals for the system and that would change from time to time as management would decide they wanted to do this or take that direction or try to implement this new approach.

So everything was subject to change and that was the way management liked it because you know up to that point you had these VP's generating products there at xxxx [company] kinda ah on the sly under their own management doing whatever they wanted to do in a loosy-goosy type of operating environment.

I: So was it also a problem with knowing who was the boss?

We had these two VPs, and of course they expected to be able to walk into the engineering room and if they had an idea or some change they wanted to implement, they expected to be heard, and have it implemented if they felt strongly enough about it.

I: Did that cause any severe problems?

P: Oh yeah. Yeah, it caused, what it does, it makes the engineering design effort less efficient because instead of doing your homework up front and knowing what goals and what specifications you are trying to achieve, you do it on a day to day, or weekly, or monthly basis so everything is always in a state of flux. And the result is that your engineering effort is much less efficient, you take longer to get things done, you end up changing and redoing and backtracking, in the design effort and the design effort stretches out and gets more expensive.

Here the project manager was lamenting how these two vice president/owners were both

participating informally in the project by experimenting with their own enhancements and adding

their own features ad hoc throughout the development process. While their status as owners

allowed them the ability to force design changes and product enhancements, the atmosphere was

described as generally more cooperative and friendly with the team members and owners on a

first name basis. Nevertheless, the issue of establishing and limiting the scope of the project was seen as an ongoing struggle that often went unresolved. 
While priorities and scope changes were often contested and sometimes went unresolved during projects, other types of differences were also problematic. Several project managers described their role as a negotiator to resolve differences among the project members or stakeholders. In negotiating, the participants took an active role in attempting to persuade other team members that their particular interpretation of the project situation was more valid and productive. These situations were attempts at collective sensemaking, in that the stakeholders were attempting to gain consensus of meaning for the pending project decisions.

Negotiating solutions. When participants talked about whether their projects succeeded, they often commented on the process of negotiation whereby team members attempted to resolve differences about their different perspectives on the project. When project managers discussed their role as negotiators, it was quickly apparent that they were referring to dealing with a variety of project issues outside of the technical arena. For example, in the projects studied, the project managers did not always have control over their project budget and resources. In the words of one PM who had to consistently negotiate among several stakeholders for resources:

I: So, normally I think the project manager usually has control over all of the resources.

P: At least that's the goal. Of course in real life it doesn't always work out that way. It's like PMI always gives you this ideal situation, that the PM has control over everything from budget to resources, of course that's never true. So part of what I've learned with this conflict within a team is when to let go. When to fold your hand, because we're all poker players. And when to say ok. And you know the benefit of taking that attitude, not everybody does, but I work very hard at it to negotiate effectively, to trade off some things for others. If you are the PM and in an ideal world you would have complete control over the budget and all that, but then in our world, you have outside PMs who come in and scrutinize your work, and there's disagreement of opinion which is very likely. The outside PMs will win. So you have to negotiate for what you really need before hand and you have to know what battles you're willing to lose.

The attitude and the benefit of being non-confrontational that this project manager was referring to involved constantly working on relationships to smooth over problems that might occur in the future and to gain social capital for later exchange. Also, this was an institution where the project 
funding came from an external organization that brought in outside project managers to have oversight and review. Therefore, resource availability and control involved the good will of a number of stakeholders and outside reviewers. Consequently, for this project manager, negotiating was carried out within the framework of ongoing relationships and held benefits that extended past a specific project. Again, sensemaking often took on a broader more contextual role in the negotiation process. Because these team members and clients would have future involvement, there were both short and long term benefits to consider in coming to consensus about the immediate project issues.

Another type of conflict occurred when unresolved differences among project sponsors became an ongoing problem for the project manager who was charged with developing a project plan and obtaining agreement for a specific course of action.

I: So, outside of the website, did these investors come together on the other decisions?

P: No, as a matter of fact it was bizarre for me, because I've never been in that environment before. I remember being called into a meeting in $\mathrm{xxxx}$ [city] and the CEO would pick up his conference phone and call the other investors. And they would just have a shouting match in front of everybody about how this thing should be run. I've never experienced anything like that, but here you have people who each by themselves are running million dollar companies all with slightly different ideas about how this thing should be run. But one guy is the acting chief and if he doesn't like the results, he tells the others put up or shut up. So it was very difficult for me personally to try to work in an environment like that where there was no one singing in concert. Like I said there were lots of chiefs, lots of people wanting different things to happen.

In this example, conflicts emerged among the sponsors as they expressed their differences in allocating their marketing budget to launch their product. The project manager reported that obtaining consensus was an ongoing problem which resulted in substantial project delays and added costs. Ultimately, the owners failed to reach agreements on their differences and the project manager was required to implement a compromise solution. This resulted in two different marketing plans to be implemented, greatly increasing the total cost and diffusing the efforts of promoting the product. 
Another issue in project negotiation is the connection of projects to their larger

organizational contexts. That is, the goals of projects within organizations may conflict with larger organizational goals and require the participants to resolve their differences through bargaining. This is especially apparent in the next example, where an internal project sponsored by the quality department in a medium-sized manufacturing organization was perceived to interfere with the goals of other departments. The 'customers' for this project were identified as each department within the organization that all had to participate by contributing resources and would also benefit in the achievement of company wide ISO certification. Achieving ISO certification was primarily perceived to be a marketing feature, which would communicate improved product quality to their customers. Cooperation was described as limited because the organizational departments were reluctant to divert needed resources to a project that they would not receive recognition for. In contrast, the project manager was responsible for overcoming this by negotiating with each department for their efforts.

I: So what is the major problem?

P: When I contact the representatives of the other departments, they say they don't have time, or they don't know what ISO is about or they don't want to participate. And there is no enforcement from the top management or their management. Basically, I am alone trying to do something, it's not like the whole company is doing it.

I: So you depend on other people cooperating with you?

P: Yeah, yeah, and I can't influence them because they don't report to me. We have other projects in $\mathrm{xxxxx}$ [city] and they have not been cooperating at all, they just ignored all the emails and phone calls, and everything.

I: So you call and they won’t return your calls?

P: Yeah, they're not saying “no," it's just passive resistance. And like tomorrow, I have to fly to $\mathrm{xxxx}$ [city] and I've got three weeks scheduled, and I'm setting up meetings and everything, and I got a call that one of the engineers there had to leave, and so my manager said "oh, let's cancel it because she is out of the office.” But, we have a deadline in May, and I'm just going up there and typically only like half of the team will attend the meetings anyway. And if I cancel this meeting I don't know how we could ever get the project done. 
Clearly, the various customers within this organization had other priorities which threatened the success of this project and made it more complex and difficult to implement. What this participant sees as passive resistance may result from the inability for the project sponsor and project manager to negotiate the priority of this project within the organization and communicate that to the others. That is, the failure of collective sensemaking to occur regarding the importance of the project to the individual departments. This is not to suggest that the employees are not familiar with the project and its importance. The project manager was clear that numerous postings on the corporate intranet and the information presented in organizational meetings ensured that everyone was familiar with the project and its objectives. However, these employees were not officially tasked with cooperating, and resisted participation in activities that interfered with their other work.

This final example of difficulties in negotiating solutions to project problems began with an oversight in estimating the cost of customizing a software product for a unique application. The problem remained undetected until after the contract was signed and the delivery dates were established. When the project manager discovered this problem he spent much of the first two months seeking technical solutions within the project team. When it became clear that no technical solutions were forthcoming, he turned his attention to negotiating a solution with the customer.

P: Well, I [the PM] had worked with [name omitted - the client project manager] for a long time and we had a pretty good friendship. I told him the truth, that we didn’t include this in our cost, but proposed another solution.

I: What was that?

P: For this location, we would give them a standard off-the-shelf software package and they would have to give up some control points [access control points controlled by the security system], but I tried to persuade them that there would be several benefits to them. Like, no special training, standard documentation, and no special software to maintain. Also, I just asked him for a personal favor.

I: What was that? 
P: That they just accept the change and not make a big deal out of it. Because if they did, it would swamp us.

\section{I: And so what happened?}

P: Well, he reluctantly agreed. I gave them a letter with the basic agreement on it, and asked them to sign it and fax it back to me. Well, they did. I was holding my breath until we got it, but it came through. Once we had it, we were totally off the hook. It saved us. But the management never knew how much trouble we would have been in if we had to deliver what the sales guy actually promised.

Clearly, this negotiation was based on the personal relationship of the participants. The supplier was surprisingly open about the situation and held little if anything from the client. He went on to describe how they explored a number of areas where the supplier could provide additional value in compensation for the features that would have to be forgone. This project, as spelled out in the contract was described by the participant as “doomed for failure.” Technically, the specified requirements could not be met with the resources available and, even with added resources, the final product would exceed the customer's desired delivery date by several months. While this project ended successfully, it apparently hinged on the ability for two stakeholders to first negotiate among themselves and then adopt changes that were plausible to their organizations. For the client this meant that the tradeoffs between the two system variants had to be framed adequately for the change to be approved by his organization.

Overall, effective negotiation was an important reoccurring theme that participants posited for the ultimate success or failure of their projects. They consistently indicated that while important disagreements and differences were common in projects, the inability to effectively negotiate solutions would lead toward additional costs, schedule delays, or complete failure.

Along with adapting to changes and agreeing on priorities and scope, effective negotiation represented the third form of unresolved conflict found in this study. That is, these topics became problematic when they were ongoing and involved central issues, which were frequently discussed but resistant to solutions. For these conflicts, the participants routinely talked about their own positions and the positions of other as the expended considerable efforts in 
trying to persuade their counterparts to their view. In this way, these forms of unresolved conflict were primarily based on issues with collective sensemaking in that the construction of meaning was primarily a group process that occurred in meetings and among individuals. Successful negotiation could then be equated to effective sensemaking at the collective level.

\section{Summary}

The present study has approached the topic of conflict in high technology projects from an interpretive perspective to better account for the specific context and the environmental influences that may contribute to actual project experiences. In that regard, it paid close attention to two factors that have been implicated in project outcomes. That is, the study investigated how project complexity and the ambiguity of success can be important factors in project communication. The findings indicated that each of these can contribute to project conflict in a variety of related ways.

Complexity made projects more ambiguous, especially as complexity was defined in broader terms than the task-related activities and incorporated personal, organizational, and environmental factors. Some of these contextual factors were hidden or proscribed from communication by individuals who invoked their power to shape conversations, while other factors were simply unobserved by the project leadership until they came to have significant influences on the project. Often, several forms of complexity came together simultaneously to provide further opportunities for conflict. Similarly, the ambiguity of success was more pronounced than I expected, and was often dynamically changing throughout the project. In some cases, this was directly related to and became the consequence of project complexity. That is, when some projects became more complex, the definition of success was changed to help make the projects more successful. In addition, there were often different perspectives within the same project on what would constitute success, which served to construct conflict through the planning of incongruous activities in support of disparate views of success. In all, the two factors of complexity and success ambiguity provided an important contextual backdrop from 
which to view project conflict and investigate its variations and impact on high technology projects.

In terms of conflict itself, the results of the current study revealed three types of conflict that were perceived by the participants to exist and be significant in their high technology projects. Unexposed conflicts were those where latent differences became problematic when they surfaced. These were issues with knowledge and ambiguity, often exacerbated by ongoing failures for the stakeholders to communicate about even basic issues relating to the project. However, this problem also included incompatible differences in meaning that, when unabated, had a significant project impact. For this type of conflict, sensemaking was primarily an individual process, as the participants lacked access to important common cues that could offer the potential to bring them together.

The second type of conflict was represented by unexplored conflicts, which primarily represented issues with power and voice. In this type of conflict, the participants understood that their communication was constrained and there were unwritten rules about what topics were open for discussion. In these cases, they generally recognized that the existence of power that constrained their actions, and perceived the consequences of ignoring that power. For this type of conflict, sensemaking appeared to be both an individual and a group process. That is, the participants used both their individual and group resources to construct meaning for incongruous events that were not expected to occur in their projects. However, power was also shown to be diffused and originate from multiple sources. In this way, the influence of power in project activities was somewhat more complex than would be suggested by traditional views of a hierarchal organization.

The third type of conflict was designated as unresolved conflict to emphasize its open nature about issues that were continually in dispute among at least some of the project stakeholders. This most closely resembled traditional views of conflict where parties are openly debating their differences such that onlookers and more peripheral members can articulate the 
opposing positions and arguments. These were conflicts that became significant because they were enduring and could not be resolved by the team resulting in missed opportunities. In other cases, projects were placed on 'hold' or encountered considerable delays because a decision or consensus could not be reached. This type of conflict was characterized by collective sensemaking, where opposing individuals devoted considerable time to discussing the dispute in meetings and among themselves. Such conflicts also became readily intractable as the participants adopted 'positions' that became more strongly entrenched over time.

Overall, the data provided here represented the contribution from 36 participants who participated in in-depth interviews along with numerous others who provided valuable information as part of the participant-observation sessions. Collectively, they provided important evidence to support three distinct types of conflict that can emerge in high technology projects and become problematic when left unabated. The following section provides a more detailed discussion of these findings and their consequences for high technology projects. 


\section{CHAPTER 5}

\section{Discussion}

The present study identified certain communication processes in high technology projects that are indicative of eventual project failures. Specifically, it has explored the role of conflict in projects, where conflict is reflective of important differences among the participants that become problematic when they remain unabated. It differs from most project management research which has primarily taken a positivist stance (Bredillet, 2004) resulting in assumptions that deemphasize contextual differences and presume that projects embody relatively stable deterministic systems (Winch, 2004) fully open to functional analysis and managerial control. Rather, the current approach follows Ivory and Alderman (2005) in their assertion that:

Live projects are themselves social and technical systems that have been designed to achieve particular social or technological goals. Projects, like more permanent systems, are "designed" based upon assumptions (and sometimes more tentative theories) about human, organizational and technical behavior. Problematically, these assumptions and theories can and do break down in face of the complex social and technical realities (p. 6).

Thus, the more holistic, context rich, interpretative approach provided here begins to address the limitations of functionalist studies to develop a more comprehensive basis for explaining the communication and organizational behaviors in complex projects. To that end, the present study has centered on the interaction of social and technical processes that create an atmosphere for project problems to arise and go unabated until project failure. As partially articulated by one study participant, these processes are essentially communication processes, and operate to construct and tie together the environment, organize the participants, and define the outcomes. As he recalled,

Where we generally fail and it is general .... in my company and probably in other companies, we fail in communicating. That's probably the major reason why it [a project] will fail. Because communication is not there. And what is communication? It is creating the same vision, the same reality. And why is communication not there? Because sometimes interests are somewhat different from one individual to another. It is not addressing why my goal is here and your goal is going in another direction, --- then it will 
fail. And it is often seen as bad management that has nobody together, and not seeing inside of things to understand what is happening and so on. But it is communication.

This participant nicely captured the spirit of this study which was to investigate how prolonged conflict in projects could exist as a precursor to project failure. For this owner of a high technology company who managed two to three projects at any given time, project conflict was about misaligned meanings that prevented consensus among team members. In the present study, the focus on communication was extended to include other forms of intractable conflict that occur in projects which might be associated with their outcomes. To address this, the study used a qualitative approach to discover the various forms of conflict which actually occurred in projects and to identify those types that had a consequential impact on project outcomes. By utilizing a constructionist paradigm, this study incorporated context as an essential component to how conflict emerges and becomes enduring in projects. This approach is consistent with the work of Belassi and Tukel (1996) who provided an important insight for those studying project management effectiveness. That is, they began to ask not just what variables were responsible for critical project success and failure, but how those variables might interact during actual project activities. While their methods limited their ability to document the emerging and changing nature of successful project behaviors, their work signaled an important change for future research. Essentially, they suggested that research needs to be cognizant of both the interactional processes and the dynamic effects of multiple project influences as they uniquely come together in specific projects.

This study took up that challenge with its methodological focus that integrates the various influences that individuals experience in projects and does so from their own perspective. In this way, it differs from many studies on organizational topics by approaching project conditions and conflict as socially constructed by the participants rather than being derived from fixed characteristics attributed to individuals or organizations. When compared to traditional views of conflict, this approach to understanding the conflict experiences of project stakeholders can 
provide richer sources of information that reflect more substantial project related issues and problems. The broader perspective developed here operates very differently by focusing on how individuals construct meaning from their own perceptions. This follows Litterer (1966) who suggested that in addition to the traditional view that conflict is a competition over scarce resources, it can also occur from perceptual differences resulting from peoples’ own experiences and perspectives. He notes that "it is frequently observed that people in different functional departments will tend to have different views of what is good for the company and how things are to be done” (p. 183). This was also found among the participants of these high technology projects who experienced conflict from their disparate perspectives in project teams. Their experiences are reflected in the following discussion of the research findings.

\section{Answering the Research Questions}

$R Q 1$

The first research question addressed how project complexity contributed to the conflicts that foreshadow project failures. Some insights to this question were found early in the study as participants frequently referred to the complexity of their projects and perceived the complexity to be responsible for many of the problems that they faced. The idea that complexity itself may be sufficient for project failure has been observed by a number of researchers. For example, Ivory and Alderman (2005) suggested that:

by drawing on a wide range of literature on failure of complex systems, that failures in projects can be similarly understood in terms of complexity. Such literature suggests that failures occur as a consequence of the multiple interactions, internal contradictions, and geographically dispersed and multinodal nature of projects (p. 5).

Also, Jaafari, Doloi, and Gunaratnam (2004) have noted that the ability for project teams to complete their projects successfully, dramatically decreases as their projects get larger and involve more complex relational networks among the participants. This was consistent with the current study where relational networks and organizational complexity were two of several variables which worked to increase the ambiguity and uncertainty associated with projects and 
provided more opportunities for disparate sensemaking (Weick, 1995) among project members throughout their projects. While Jaafari, Doloi, and Gunaratnam did not label their interaction difficulty findings as conflict, it nicely fits the constructionist approach of the present study which posits that conflict emerges from incongruous meanings that are developed by project members as they engage in project activities that have mutually dependent consequences. As demonstrated by the findings, these differences of meaning may be latent (unexposed conflict), suppressed (unexplored conflict), or they may be openly debated (unresolved conflict). However, for each of these conflict types, complexity operated to make sensemaking more difficult by enriching ambiguity and providing additional cues for varying interpretations. In addition, because the project members occupied unique positions in their projects, they brought their own experiences and perspectives into their interpretations, adding to the effects of project complexity as a backdrop for conflict to emerge. Thus, the contextual aspects of the project environment contributed substantially to this complexity and the increased difficulty for the participants to achieve some degree of coherency in their sensemaking efforts.

The PMBOK (PMI, 2005a) along with numerous management texts (Berkun, 2005) portray project complexity as a potential source of problems that can be systematically addressed by adequate planning, breaking down tasks into smaller units, clearly identifying deliverables, developing adequate risk plans, etc. While all of these strategies were present in the projects studied and frequently discussed by the project participants as having an important place in project management practices, there was little consensus that these techniques were robust solutions to project failure. Rather, project complexity was seen more holistically and associated with evolving knowledge, organizational changes, competing interests, personal relationships, past history, and the like. Essentially, the project managers were consistent in their assessments that project complexity was ongoing and enmeshed with organizational factors as well as the environmental context where the actions of competitors, global concerns, and international regulations came into play. Thus, project complexity was consistently seen as an ongoing factor 
that was constantly unfolding rather than a set of risks that could be known in advance and dealt with through adequate planning and appropriate risk management.

The topic of risk management is worthy of noting due to its popularity as a remedy for the dangers associated with project complexity. It was almost always raised by the participants as the appropriate solution for project complexity, yet, they seldom found it effective in their own projects. As discussed in the literature review, risk management is heavily emphasized in the PMBOK and other texts as a planning tool and a process that is intended to address project complexity by identifying potential problems and circumventing them. Yet, several participants indicated that the risk management component in their projects (both the successful ones and the unsuccessful ones) was not sufficiently developed or resourced to account for the actual risk and complexity of their projects. In addition, they concurred with the assertions of Winch (2004) and Williams (2005) described earlier, that real projects operate in a much less deterministic environment where a number of serious issues may arise that cannot be known in advance and planned for. Consequently, the participants of this study often cautioned about over-reliance on risk plans, as they felt that such contingencies were far less effective than generally posited. According to one project manager “the problem is, you don’t know what you don't know” meaning that only identifiable risks can be accounted for in advance. He went on to explain how adequate risk management would not just require that a risk be identified, but also exactly how it occurs, when it occurs, and be inclusive of the related project, organizational, and environmental variables that coincide with the occurrence. He reported that to be adequate, such risk planning would be far larger than the projects themselves. For his own organization, he indicated that because of limited resources available for risk planning, it typically involved an emphasis on the most obvious risks that had feasible contingencies rather than those that were the most potentially serious. Several participants echoed this view:

P: In our organization, you have to have a risk plan for every project, it's part of the required process. So, we identify those things that we can think of, we make a list, we work through how to solve them. The weak part is 
that it mainly turns out to be the easy ones that are well documented. And, all this really happens in the beginning, before everyone is busy working on their tasks. The problem is that the project conditions change every day. Nobody goes back and really looks at how the conditions really affect the risk plan, and what new risks become part of the project. Everybody pretends that it happens, there's always an agenda item on the project meetings that says "are the risks still valid?", but does that really mean anything? No.

The extent to which risk plans were helpful for these participants was difficult to ascertain. While they clearly were not seen as robust solutions to their project problems, they could have played a more subtle role in helping the participants focus their initial efforts toward more successful project strategies. However, while numerous stories unfolded about adapting to unexpected changes, delaying a feature to a future release, or negotiating for a contractual change, there was a stark absence of talk about utilizing established risk plans to solve important project problems by implementing a pre-planned contingency.

The contribution of project complexity to conflict was found specifically in the mechanism of sensemaking (Weick, 1995). The participants reported how the numerous sources of partial and incomplete information came into their awareness throughout their projects. The information varied by source, type, completeness, perceived accuracy, and numerous other factors. As part of the sensemaking process, the participants had their own methods of evaluating these segments of information, utilizing factors such as their own backgrounds, needs, relationships and interests in the project. They were especially prone to use their prior project experiences to diagnose their current situations, even when those prior experiences were remarkable different from their current project. Taken together, the aspects of project complexity provided a vast and rich source of ambiguous cues that were exacerbated by the inherent complexity of the technology itself. This became the backdrop for conflict to emerge in these projects as the participants worked toward achieving project success. However, as discussed in the literature review, the concept of success itself was also problematic and subject to 
sensemaking differences. Therefore, this was also an important topic in the present study, as reflected by the second research question.

RQ2

The second research question directly addressed how project members understand project success. This is an important topic for the current study and addresses a serious discrepancy that has emerged between the traditional view (PMI, 2005a) that project success is deterministic and easily defined (in terms of cost, quality, time), and the findings of researchers who argue that in actual projects, success is often ambiguous and evolving (Avots, 1984; Belassi \& Tukel, 1996; Briner et al., 1990; Kleim \& Landin, 1992; Pinto \& Mantel, 1990). This second, more complex and emergent view of project success was supported by the findings of the current study where the participants talked at length about their own criteria for success. While the participants had unique experiences and occupied varying roles in their projects that influenced their perceptions of success, what was most compelling was the emergence of three different perspectives for project success. First, for some, success was seen as the ability for the organizational and project management to achieve their “actual” objectives, which may or may not remain consistent with the project charter. For those organizations that exhibited a military-like or heavily authoritarian structure, the management voice was stronger than any process or written objectives. While these projects often had fully developed project plans and very formal "acceptance” criteria, these participants indicated that the real criteria for success was simply "pleasing the boss.” That is, additional unwritten requirements could be added, or any of the listed criteria could be ignored by the dictate of the executive. This finding has some support in the literature from those who argue that once started, projects are not managed to the stated goals of the project, but rather to wider organizational objectives. For example, according to Ives (2005) actual projects are typically managed to satisfy four different factors. These are (1) impact on management overhead, not project effectiveness; (2) gain executive willingness to adequately sponsor and stand behind 
projects; (3) constrain project scope changes, and (4) ensure that project sponsors retain authority. For the participants whose organizations adopted these criteria, such practices were described as consistently practiced and part of the culture. However, most participants described the criteria for success from one of the other two perspectives.

The second perspective toward success was represented by participants whose organizations held to the traditional ideas about delivering projects to specific functionality, cost, and delivery objectives. However, organizations varied considerably in which of the three objectives were most valued as well as how much the criteria was allowed to change. For instance, in the regulated utility example, the ability to obtain cost recovery was entirely a function of the project being implemented and shown to be performing its intended function. If funds were expended and the project was canceled for almost any reason, the cost had to be absorbed by the utility. However, even if the project was vastly over cost, that cost was eventually recoverable as long as the end product was being used for its intended purpose. This is exactly what happened in the customer service software project that was described in this study. It had been established as a two year project, but escalated to five years before it was completed and deemed to be a success.

Other organizations that held to this functional view of success would prioritize project time or cost. For example, the rapid change of technology made very fast time-to-market an overriding criterion for the telecom projects described in this study. In the telecom example, the organization chose not to adapt their project to respond to competitive technology on the belief that time-to-market was the more critical success factor, although in retrospect, this appeared to be a serious miscalculation leading to the project's failure.

The third perspective was reflected in projects where immediate and long term customer satisfaction generally took precedence over specific deliverables. These tended to be IT implementation projects or service projects where a specific customer was identified, rather than development projects where multiple and often anonymous customers were the beneficiaries. For 
these projects, the concept of success began with specific stated objectives which formed the basis of what was to be delivered. But these projects were characterized by the impact of personal relationships which could have great sway over the ultimate attribution of success. Perhaps the most common theme in stories about how these projects transpired was the informal agreements that accompanied the official project plans. There was often considerable discretion that could be exercised among the participants to resolve problems and declare success when that became important for both parties. In that regard, the participants often had other motivations to be successful that influenced their judgments. For instance, several organizations had adopted a strong emphasis on individual accountability which would equate project failure with employee failure. One study participant from such an organization described how the culture of individual accountability pervaded the entire company causing team members to distance themselves from floundering projects. This went as far as removing their names from lists and not "officially" attending meetings. In contrast, there was such a prominent recognition system for successful projects that team members suddenly jumped on ship when the project looked successful. This also became more personal as individuals would receive financial incentives for their participation in successful projects. For project managers, in addition to incentive pay, their reputations were often tied to the perceived outcome of their last project. Several participants described how this orientation toward success created an interdependent atmosphere where the team members negotiated the success criteria among themselves.

For some participants, the personal relationships that they had developed in their projects created an incentive to categorize the project as successful exclusive of other factors. As these participants became more and more interdependent, the possibility of failure was also perceived to be relationally threatening. For this study, the most interesting example of this was on an IT implementation project where the project managers from the developer and client sides became such close friends that they went on a vacation together. Their close friendship was maintained throughout the project which reportedly had a substantially positive effect on project activities 
including their negotiation and problem solving efforts to ultimately make the project successful. However, the participant did disclose that some of the features promised in the scope of work were not delivered until the next phase.

Overall, the results of the present study provide evidence for three orientations toward project success, which might be labeled as the (1) power perspective, where project success is declaratory from those who exert control over the project environment; (2) functional perspective, where success equates to the traditional assessment of features, cost, and time; and (3) relational perspective where personal relationships and long term business relationships have a substantial influence on how the outcome is framed and labeled. While these reflected very different project experiences for the participants, the common thread was that all of the participants had a strong reluctance to label a project as "failed" and this appeared to extend to their organizations as well.

Taken together, these examples illustrate how the perception and management of "success" was a non-trivial aspect of the projects in this study. Not withstanding that the criteria for successful projects was problematic in the current study, its primary purpose was to discover how conflict might be a precursor for project failure. It turned out that while success and failure were highly manipulated terms when used by the participants in their official capacity, they also maintained their own common sense notions about which of their projects failed. That is, the participants knew when their projects failed and could easily contrast them with their more successful projects. This provided a robust dichotomy for inquiry about the effects of project conflict from the perspectives of the participants and within the context of their own environments. This is the basis for the third research question, which attempted to discover more about the forms of conflict that might be seen by project participants as affecting project outcomes.

RQ3

The third research question specifically addressed the role of conflict in high technology projects by seeking to identify what types of conflict were perceived to affect project outcomes. 
By taking a constructionist perspective, this inquiry was open to how conflict might be constructed socially through the communication of the participants as they made sense of their situations. This differs from traditional research on conflict that emphasizes the competition for scarce resources, which has dominated thinking about how conflict does and should operate in organizations and within projects. That is, conflict has often been seen from a functional perspective which locates conflict as a personality construct or leadership weakness that is almost universally a negative influence in projects. From this perspective, conflict has little to do with the situation, except that certain triggers may elicit conflict from those prone to possess it. Other traditional views of conflict suggest that certain types of communication messages, for example, aggressive messages are responsible for conflict. In contrast, the present study found conflict to be multifaceted and mostly a communication constructed phenomenon that is contingent on the specific project environment and the sensemaking processes of the participants. In that regard, there were three characteristically different types of conflict that were derived from the reported experiences of the participants as they reported on their high technology projects.

First, participants described unexposed conflicts as a latent form of conflict, which is represented by ongoing issues with knowledge and ambiguity about important potential differences in the projects. In general, this category represented a failure to communicate sufficiently to both uncover and understand important differences that would later become problematic for the projects' success. That is, in some cases there were insufficient conversations to convey important information, such as project requirements, team member needs, or organizational processes. However, a more subtle form of this conflict was that the participants would develop different interpretations for the information that they possessed, resulting in incongruous beliefs or actions that often remained undiscovered until they became seriously problematic later in the projects. This was exemplified in the story about the two companies that were co-developing a redundant navigation system and failed to discover their different perceptions about the responsibility for system integration until the project ran out of time and, 
thus, failed. In this example, there was abundant communication between the supplier and the customer resulting in a multiple page contract and statement of work, along with detailed acceptance procedures. However, the conversations and resultant documentation failed to uncover the disparate meanings that each party held for their position. This was not a simple oversight or misunderstanding, but rather the result of incongruous sensemaking where each party found abundant but disparate cues that supported their beliefs in what the agreement was about. Consequently, for both parties, their level of uncertainty was reduced sufficiently to not pursue additional clarification. This lack of further communication should not be interpreted as conflict avoidance, because their differences were latent at that point.

When conversations are not held about perceived differences, the traditional conflict literature has often framed this situation as a ‘conflict avoidance' style. Studies have presumed that individuals avoid communicating to escape an uncomfortable interaction, especially if the partner is known to hold incompatible viewpoints or possess undesirable conflict characteristics (e.g. verbal aggressiveness). In contrast, for unexposed conflicts, the participants were not specifically trying to avoid interactions with their partners, but may not have the access to them, or as the example above indicates, may not comprehend how they have constructed incompatible meanings for their situation.

However, even with latent differences, there was an indication that some project managers anticipated and worried about the existence of incompatible meanings with their customers or clients. This was clearly revealed by one project manager in this discussion.

I: What is your biggest concern in managing projects?

P: That's easy. I lay awake at nights wondering what questions I didn't ask. I wonder what surprise is awaiting me the next morning. I wonder when a customer is going to tell me "we can’t use your product." Have you ever heard the saying “you don't know - what you don't know?” I live in fear of that - not picking up on a change, not understanding what someone was really telling me, not finding out that one key piece of information that will changing the entire project.

I: So how does that affect what you do every day? 
P: I think it makes me second guess everything I'm told. It's not that I don't trust my team, but I try to listen for every little nuance, I keep asking myself "am I hearing something that just doesn't sound right, something that doesn't fit with what I believe to be true?” But at the end of the day, there is always something that you didn't know about, didn't ask, you just hope you can react to it and deal with it before it kills you.

Similar comments were made by other project managers who felt anxiety about unknown differences that were in conflict with their project plans. In some cases, they managed this by communicating more frequently or by distributing more written documents. Overall, the project managers did not perceive the unknown differences as conflict but admitted to frequently being worried about the discovery of unexpected information and events that presented major problems to their projects.

For most participants, conflict was perceived to be the loud or angry disagreements about contested issues. It was often seen as unprofessional and something to be avoided. However, when asked about being surprised by unexpected information and events, they talked at length about the differences between what their perceptions were and what they were surprised to discover. While many of their surprise discoveries were pleasant, it was more common to hear about the unexpected emergence of serious problems.

Some participants who managed IT projects recalled how they attempted to avoid the problem of unpleasant surprises with their end user by performing early prototyping. This technique is one where the programmers create simulated output screens to show the user exactly how the end product will look. This improves communication by providing an early opportunity for their customers to provide feedback about the intended operation of the system. However, this technique is limited to the most obvious functionality and fails to deal with more obscure features (e.g. application response time) as well as concerns from other stakeholders.

The eventual problems which emerge from unexposed conflicts may parallel the limitations reported with performing adequate risk management in projects. That is, because it is only the identified risks that are open for assessment, unknown risks are not communicated or 
planned for. In both cases, the lack of communication about critical information is not available for sensemaking and creates the latent potential for project failure. However, with risk management, these potential issues are contingencies which may be unlikely to occur. In contrast, unexposed conflicts represent different and potentially incompatible perspectives that may be central to the actual project features.

From a theoretical perspective, in unexposed conflict, sensemaking is prone to failure because cues are for the most part individually selected without the opportunity or emphasis for collective review. Thus, there is little opportunity for co-orientation as individuals rely on their own perspectives and project their needs on others. This conflict type was clearly identifiable because of its latent form and, therefore, was often retrospectively defined. However, the participants seemed to suggest that they frequently had intuition about this type of conflict, knowing that too little communication was occurring with their customer or recognizing that they were making assumptions without sufficient justification. In this way, unexposed conflict contrasts with the other types, because in both unexplored conflict and unresolved conflict, the participants could clearly articulate the issue and its potential impact on their project.

The second type of conflict is designated unexplored conflict, which represented issues with power and voice. For these conflicts, the participants recognized that important differences existed, but felt that other factors prevented an open discussion of the problem. Because certain topics were proscribed, the participants often portrayed an attitude of working around the system or using informal communication networks to seek information that they needed to understand and respond to their situation. In other cases, the participants reported a sense of resignation, accepting that forces beyond their control reduced their discretionary activities. Often, the participants discovered these sources of power when they found that their projects were being actively interfered with by outside forces. This type of conflict could be modeled with classic sensemaking in that a hidden source of power appeared as a "shock" (Weick, 1995) that initiated the sensemaking process. That is, project managers would discover a contradiction in the policies 
or procedures established for their project when compared to their directives. For example, two different project managers reported their discovery of an alternative project with incongruent goals to their own projects. These competing projects were hidden and, once discovered, represented a shock that initiated sensemaking activities by the project managers in defense of their own projects. However, in both situations, their ability to understand the impact of these alternative projects was inhibited due to the proscribed nature of the topic and the cultural norms of the organization. As a consequence, sensemaking cues were limited because of access to individuals and the topics that were allowed to be discussed.

The third type of conflict was identified as unresolved conflict and represented issues with action and timing. For this type of conflict, the participants openly discussed and sometimes fought over topics such as adapting to change, setting priorities, and committing to specific decisions. These were conflicts that were well known and rehearsed and the participants could describe their own position and the positions of others. While the topics included both central and peripheral issues, there was often one specific issue that served as a roadblock on the project. Here, sensemaking was primarily a collective process, which transpired in meetings and discussion of the topics. Opportunities for co-orientation were frequent as participants reported having conversations about contested meanings, which in some cases, spawned additional tasks to gather the required clarifying information. In other cases, these discussions became negotiations where the participants attempted to work out solutions that 'made sense' for the team. The participants provided several examples where unresolved conflict became protracted and problematic toward progress, resulting in missed opportunities or actual project failures.

Overall, while these three types of conflict are conceptually distinct, they sometimes occurred together in projects or one type evolved into another. For example, one participant described how she was allowed almost no contact with the customer (unexplored conflict) until the conclusion of a ten month long development project. This represented an ongoing unexposed conflict until the implementation phase when she discovered that this product would not work at 
the customer site. Then, the conflict became unresolved because the cost to provide the needed solution was untenable for both the supplier and customer and no other solutions appeared plausible to the parties. The project was then canceled. In this example, each of these conflicts may have contributed to the project failing. Access to the client and better information about requirements may have allowed the project to continue successfully. However, there may also have been the potential for negotiated solutions that were not achieved at the end of the project. Thus, the degree to which conflict in its various forms may combine to operate as a precursor to project failure is an important factor to consider. This is reflected in the final research question, which attempts to also draw connections between project conflict and project outcomes. Consistent with the qualitative methods used, the relationship between conflict and project failure is not a causal claim, but rather a theoretical argument supported by the participants' own experiences. The intent, as outlined in RQ4, is to identify certain types of communication conflict patterns that are consistent with problematic trajectories. More specifically, the communication patterns found in the current study were types of conflict that when unabated, had important consequences on the outcomes of many of the projects investigated. To be clear, it was not simply the existence of these types of conflict, but rather how they emerged around important differences and became enduring, that is posited to be a constitutive force in problematic projects. Thus, the final research question dealt directly with the issue of how enduring conflict that exists during a project may be indicative of eventual project failure.

RQ4

While various types of conflict have been shown to emerge and become problematic in projects, RQ4 calls for evidence that enduring conflict may be indicative of eventual project failure. This question was made substantially more difficult because of the findings presented earlier and discussed in RQ2 regarding project success. In contrast to the concept of neatly and cleanly defined success criteria promulgated by management texts and standards’ organizations, the present study found strong evidence that the nature of success itself was highly problematic 
and often evolved throughout the projects. In addition, there was little evidence for a unitary assessment of success and in some cases little consensus for success once a project was completed. Nevertheless, most participants articulated general goals that were somewhat enduring even as specific requirements and conditions were changing. Regardless of organizational determinations of success, the participants could clearly identify from their own perspectives, which of their projects were successful and unsuccessful. In addition, the participants were often reflective on their project experiences, offering their insights about their own perceptions of factors that were important contributors to project outcomes.

In addition to the problems in defining success, the concept of conflict was also difficult for the participants to embrace. From their stories about their project experiences the participants held a fairly narrow and traditional view about conflict and initially resisted the idea that much conflict occurred in their projects. As described earlier, they tended to perceive conflict as loud arguments between individuals, which reflected a lack of skills or professional demeanor among the participants, and provided an indication of poor leadership by the project managers. Therefore, to address the broader conceptualization of conflict utilized in the current study, I turned to a number of related questions and probes, where the participants began to more openly describe the range of problems that are reported in the findings and how those problems were perceived to emerge and affect project outcomes.

To advance the argument that conflict in this broader form has important project consequences and can operate as a precursor to project outcomes, this discussion calls for additional theoretical explanation in support of the empirical findings previously discussed. To accomplish this, the following discussion integrates the theoretical perspective of sensemaking (Weick, 1995) with the findings provided by the participants of this study. Together, they provide mutually reinforcing arguments to suggest that when project conflicts become enduring they are clearly capable of detrimentally affecting project outcomes. 
From a theoretical perspective, as described in the literature review, sensemaking theory (Weick, 1995, 2001) establishes a robust framework to explain how individuals and groups develop meanings for their experiences, become publicly committed to those meanings, and then act in justification of their commitments. In this way, sensemaking is ongoing and has the propensity to generate self-fulfilling prophesies as individuals select some cues and avoid others to gain more comfort with their beliefs. As such, it has a subtle but powerful influence on what organizational members take as the 'truth’ about a particular situation. Sensemaking, then fuels different versions of the 'facts', and these versions become the basis for important project decisions and activities. However, project failure is not posited to occur simply from some erroneous input or some relatively benign misunderstanding. Rather, it is likely that multiple incongruous versions of important project information can develop and then gain inertia that ultimately creates a significant impact on project outcomes. Sensemaking provides a robust explanation for this effect by describing how a particular interpretation of information comes to dominate the thinking and actions surrounding a project. During the data analyses, sensemaking was found to operate and be associated with each of the three types of conflict that was categorized from the stories of the participants. However, sensemaking was also seen to operate somewhat differently for each type of conflict. Primarily, these differences involved how cues were developed (i.e. individually or collectively) and then utilized based on the situational context. Each of these is described next.

For unexposed conflict, sensemaking had a decidedly individual focus and clearly demonstrated how the lack of coherent meaning among stakeholders could result in incongruous actions and potential project failure as participants selected different cues based on their own perspectives. While these meaning difficulties occurred within the team and its interaction with the sponsoring organization, the most significant impact reported by the participants was the conflict that emerged between the project and the client or customer. In the most extreme examples, a product was being developed with only a vague idea of who the customer would be 
and how they would value the product. The project team then relied entirely on their own sensemaking to select the features and establish the criteria for success. Several examples demonstrated how this was a catastrophic choice for the project sponsors as this latent form of conflict became surfaced to result in a product that was unusable by the customer. In other cases, customer interaction was a significant aspect to the project, yet, the coordination efforts were ineffective at communicating the actual customer needs to the project team.

Without effective communication to coordinate their expectations, project teams and their customers often formed disparate expectations based on their own experiences and needs. Consistent with the theory of sensemaking, individuals search for additional cues that support their beliefs and their drive toward reducing uncertainty results in a plausible story that adds coherency to their understanding. In this way, uncertain beliefs are readily transformed into enduring positions that seem correct to their owners and provide little motivation to seek alternate explanations. This offers an explanation for how such projects can continue for long periods of time without these misperceptions being corrected. In fact, it suggests that as the project continues there is less likelihood for correction because the inertia for the status quo becomes stronger as individuals act in service of their beliefs. That is, as sensemaking continually operates to reify rather than challenge existing viewpoints, the confidence in existing knowledge becomes stronger. Consequently, important differences become less likely to be identified and more likely to go undetected until they cause significant problems. This was seen in a number of the development projects where uncertainties that existed early in the projects continued, but with diminishing concern by the participants as the project proceeded.

For unexplored conflicts, sensemaking occurred at both individual and collective levels as the participants attempted to operate in an environment where power effectively enabled and constrained certain project activities and exerted control over needed resources. Because many of the sources of power were not openly discussed or created obvious boundaries, the participants described how they came to make sense of the "politics” using their own insights along with 
valued advice from other associates. Also, some participants described how the operation of power was communicated through the organizational and project cultures, such that it became part of the organizational folklore. In this way, the participants understood how power might influence their projects through the emergence of both individual and collective cues. Because project managers frequently described their effectiveness in terms of establishing personal networks and negotiating for needed resources, making sense of how power operated in their organization was considered a key success factor among experienced project managers. This is consistent with Dhillon's (2003) work on power in projects, which argues that “an inability to understand power relationships during systems analysis, design, and implementation has serious implications on the well being of an organization and its business process; thus, it is a key factor in IS’s project failure” (p. 635). Several examples in the data clearly illustrated how power operated to constrain the effectiveness of projects, although I did not expect to find such diversity of power among a number of the stakeholders. This included the project manager, who was intimidated by her software programmers and, in another project, the team members who were near retirement, allowing them to resist their directives.

For unresolved conflicts, sensemaking was primarily a collective activity that was characterized as occurring during meetings or other interaction opportunities where the differences among team members were openly debated. Unresolved conflicts represented well known issues or differences of opinion that the participants held in projects. These ongoing debates were sometimes similar to the more traditional view of conflict, where the individuals knew about other interpretations but had good reasons not to agree. While power might have operated to shape the discussion, for this type of conflict it was insufficient to force a decision. Therefore, these conflicts were described as "unresolved” because of their open and ongoing nature. For these conflicts, the attempts to achieve collective sensemaking were continuously unsuccessful, as individuals failed to converge on which cues should be valued as the significant 
inputs to the sensemaking process. Consequently, important actions were often delayed until they were ineffective or, in some cases, the projects were simply suspended awaiting a consensus.

Many of the participants talked about how a "window of opportunity” was perceived to exist for unresolved conflicts. That is, when issues were well defined, the participants indicated that at least two alternatives were up for consideration and frequently one of the opportunities would have a time frame for election which would end earlier than the other. In this situation, the conflict was often described in terms of obtaining consensus before the window of opportunity closed. This also was described, albeit less frequently, for multiple alternative positions. This is not to suggest that project managers were unable to make decisions and kept their projects on schedule. Rather, some team members perceived that important information should be given more consideration or interpreted differently, and this information was deemed to be both important and perishable. This occurred primarily in the development projects where the team members differed on key project decisions during the course of their projects. For example, in one of the telecommunication projects studied, the participant indicated his concern about the features being selected for implementation during the software development phase. While he and a few other team members disagreed with the project manager's final choice, the opportunity for changes eventually closed without a consensus for adding some new features to make the product more competitive. The participant recalled how this project later failed because it lacked some of these same features that were provided in a competitor's product.

For all three types of conflict, sensemaking operated in a somewhat different manner to construct meaning for the participants. Primarily, the difference centered on whether sensemaking occurred individually or collectively and different issues emerged for each situation as summarized in Table 10. 
Table 10 - Sensemaking variations with conflict type

\begin{tabular}{lll}
\hline Conflict type & Sensemaking focus & Sensemaking problems \\
\hline Unexposed & Individual & $\begin{array}{l}\text { Lack of communication about important } \\
\text { topics }\end{array}$ \\
& Mixed & $\begin{array}{l}\text { Failure to establish coherent meanings } \\
\text { among stakeholders }\end{array}$ \\
Unexplored & Inhibited and repressed communication \\
& Atmosphere of secrecy \\
Collective & Contradictory goals \\
& & $\begin{array}{l}\text { Effectiveness of sensemaking based on } \\
\text { group skills (leadership, negotiation) }\end{array}$ \\
& Obtaining consensus within a time limit \\
\hline
\end{tabular}

What is common to all three variations, is that sensemaking provided a robust explanation for how conflict emerges, becomes enduring, and has the potential to provide a constitutive contribution to serious project problems that have consequences on project outcomes. Because the participants had little awareness of participating in a sensemaking process, they generally described their behaviors along deterministic paths, which made it more difficult to uncover the underlying assumptions that influenced their decisions. For example, the participants frequently indicated that they were working systematically and logically, often following processes that were either provided to them or ones that they developed themselves over time. They often had checklists or used software tools that stepped them through specific actions to meet their objectives. In addition, most described routine project coordination or status meetings which served to frequently (weekly or monthly) update the team, re-assess risk, and confirm whether the project was on schedule. Most significantly, they often echoed a belief that they were careful to work from "facts and data" to avoid errors due to subjective impressions. Consequently, while 
the participants recognized that serious problems existed in many of their projects that led to project failure, they were often at a loss to understand how those problems came about, why they were difficult to identify, and when they began to have problems. Because many of these individuals were trained and certified by the PMI curricula, they attributed failure to not following the prescribed processes closely enough. Yet, others (some who were also PMI certified) who relied more on their prior experiences indicated that most of their project failures were associated with either external forces beyond their control or insufficient attention to developing relationships. A third explanation is provided here, grounded in the collective project experiences of the participants. That is, that incongruous sensemaking at both the individual and collective levels has the propensity to both construct project conflicts and make them enduring through enactment and justification processes where individuals become entrenched in their own project positions. Because much of this resides under the surface in hidden assumptions and unacknowledged reasons, there may be little chance to reverse these conditions using traditional project analysis and reporting techniques.

In sum, the argument that project conflict can have serious consequences for project outcomes is well supported by the theoretical explanation of sensemaking as it applies to interdependent project behaviors. It is further strengthened by the numerous examples where the participants could clearly articulate how many of their project problems began with important differences among the stakeholders that not only went unresolved, but became enduring throughout their projects. Together, the theoretical argument along with the abundance of actual paroject examples lends strong support for the role of conflict as a precursor to project outcomes.

\section{General Discussion}

The purpose of the current study was to better understand the role of conflict in high technology projects and its consequences related to project outcomes. The topic of conflict and the qualitative approach were selected to offer a unique perspective in response to a project management culture that has become decidedly deterministic and primarily task and process 
oriented. Only recently has this trend been seriously challenged by researchers who have questioned the efficacy of a task-oriented project management model to deliver consistent results in a complex and changing environment. For example, Jaafari, Doloi, and Gunaratnam (2004) have insightfully asserted that

When projects suffer significant deviations and/or fail to live up to the sponsors' expectations, the search for the guilty party begins in earnest. Few managers question if the approaches applied were capable of recognizing and systematically incorporating the relevant project and environmental complexities, or whether the competence and capabilities of the players were adequate. (p. 143)

Such questions are infrequent challenges to standard management practices that serve to endorse taken-for-granted assumptions about the ‘best practices’ of project management.

The current study incorporated these concerns by starting with a context sensitive constructionist approach, which allowed both environmental and organizational conditions to be considered as salient factors in how actual projects are conducted and completed. In addition, this study allowed participants to speak from their own perspectives and honored their collective experiences and perceptions. Generally, these were highly tenured individuals who have been trained and in several cases certified as PMPs (project management professionals - PMI certification). Yet, they often resisted the very processes which are "officially” established to represent best practices for project management ${ }^{4}$ and frequently argued that their success should be attributed to their abilities to manipulate or bend the rules rather than to follow them.

In contrast, the current project management culture has become a powerful and largely unquestioned lobby espousing the fundamental assumption that process-oriented scientific management principles can provide 'best practices' for projects, if only the participants could be trained and carefully crafted processes can be followed. From this perspective, failures are attributable to deviating from a uniform practice that is 'known' to produce the best results. Consequently standards organizations and, specifically the Project Management Institute, has become a de-facto authority, creating standards, and then offering certification to those who learn the specific skills that are deemed ‘best practices’ for project management. However, the 
underlying foundations of these assumptions are theoretically weak and often unquestioned by its advocates. Several project managers in the current study reflected on how the PMBOK standards have become ubiquitously applied, without being critically assessed. In the words of this project manager with over 18 years of experience:

P: The PMBOK helps because it provides an orderly process, and, by the way it includes risk management. But there's a growing trend to rely on it too much.. sort of mechanically... somehow that it will make projects successful if it's followed close enough. The PMBOK was originally developed for large scale construction projects, but it's just a tool. The basics such as capture requirements, and having users sign-off on things are good no matter what the project is. But, for software projects, you have to learn how to make it work and you can't define a PMBOK process that steps you through good judgment or maturity, or tells you how to communicate with people in other countries or negotiate with all the different stakeholders, especially when you have to tell them something they don't want to hear. That's why I said earlier that it's the people issues not the technical issues that make these things fall apart in the end.

In that vein, the present study has challenged these fundamental assumptions of accepted project management rhetoric and suggests not only that 'context matters', but that specific contextual, relational, and environmental factors are precisely those that are most crucial to the success of major projects. In other words, it posits that effective management of high technology projects cannot be reduced to contextually neutral heuristics or pre-defined processes that are universally applicable to managing project tasks. That is not to say that basic process elements are not effective or appropriate, but rather, that they cannot be applied in a deterministic manner and used exclusive of the broader organizational imperatives. This is nicely illustrated by a highly experienced project manager in this study who had learned to focus heavily on the specific context and environmental factors from the very beginning of a project.

When I go into a customer account, one of the first things I ask the sales person who is the person who went in there and sold the product, is tell me about this company, tell me about the organization. Tell me who I need to be aware of. Who are my friends, who are my supporters, who are my adversaries going to be? What is the relationship between these people? Where should I spend my time building relationships? What is the strength of the sponsor for this project? Has the project been tried in this company before and failed? Are there competing projects? Do I have to be aware of someone else building a similar solution? Am I competing with them? Tell me about that. 
From her perspective, the mechanics of the project (establishing the statement of work, developing a timeline, assigning resources, etc.) were far less risky and important than the potential for failing to adequately manage the project relationships between the provider and customer. With over 25 years of consulting experience in the electronics and IT project management arena, she indicated that effective project management for large projects was primarily about crafting individual solutions for clients in rapidly changing environments. This project manager commented that inexperienced project managers often fail because they focus too heavily on the mechanics of project management without a mature understanding of the critical nature of strong organizational relationships and their potential impact on project success.

The dominant argument about establishing, standardizing, and utilizing 'best practices' in project management follows the assumption that the significant variables affecting project outcomes can be quantified and incorporated into universally applicable standards and heuristics. While many of the organizations represented in the current study utilized such standards, the participants frequently told stories of being successful in spite of the required processes rather than because of them. In fact, the skill of effective project management was rarely described in terms of knowing and following project standard practices. Rather, valued project management skills were more about uncovering problems, working around processes, and intervening in creative ways to keep projects on track. This was graphically described by one highly experienced IT project manager,

P: I've often thought there's two very distinct pieces to project management. One is the mechanics. A monkey can build a project schedule and sit in a status meeting and say "did you do it, did you not, did you do it, did you not.” But boy, it's the art of project management, which is FAR FAR more important. I think you can actually develop, I know I have, I can SMELL problems now. I can be sitting in a meeting, and someone can say something, and I don't know why at the time, but this little bell starts going off, and if I go and chase that bell, and chase that comment a little bit, and it may be nothing more than going back after the meeting and saying "you know you said blah, blah, blah, can you tell me a little more about that?” 
...It could be [that] I have a consultant who doesn't have the skills they need, and they're trying to hide that from me. It could be a customer who really isn't committed, but is playing lip service to that because his manager told him, and he is a key technician. It could be an executive sponsor who knows he's leaving the company, but hasn't bothered to make that clear to me and the team. It could be any number of things. It could be a technology issue. You know, I've had technology switched on me by development... And, in a conversation with a buddy of mine in support, they'll let slip, "you know, I was in a meeting and I heard that version 6 isn't coming out." And it's like $\mathrm{WOOOOH}$, red flag time. Then I go and chase that.

This participant clearly emphasized that this type of inquiry and follow-up was more central than peripheral to her work. For the very large IT installations that she was involved with, she felt strongly that effective project management was fundamentally about managing project relationships and developing the ability to uncover important differences that could be aggressively surfaced and resolved. Her experience and recommendations are clearly in line with the objective of the current study which has been to provide a more comprehensive platform with which to investigate the more holistic aspects of conflict in high technology projects, including the organizational, environmental, and personal considerations and their collective impacts on projects.

\section{Summary}

In sum, the present study utilized a constructionist qualitative approach to investigate the conflict in high technology projects, especially as it became problematic to project outcomes. This choice was important to adequately address the contextual factors and recognize the dynamic nature of project activities and how those activities were perceived to affect project outcomes. While this is not a new idea among organizational researchers (for example, see Kirchof \& Adams, 1989; Putnam \& Wilson, 1982; Thamhain \& Wilemon, 1975), the vast majority of project management research remains cross-sectional and quantitative in nature, with the consequence of emphasizing static and universal characteristics at the expense of accounting for contextual and environmental factors unique to individual projects. 
The data for the current study revealed multiple types of conflict which emerged and often became enduring as the participants made sense of their knowledge and committed to their actions. Consequently, many of these conflicts became indicators and constituents of significant project problems. Had the success criteria for projects been more static, many of these conflict situations would likely have been clearly predictive of project failure. However, the picture is more complex than that because the criteria for success were variable and dynamic. For the projects studied, very few organizations (as reported by the study participants) would openly admit project failure, and actively instituted a number of strategies to re-cast their projects as successful. For example, in some software projects, organizations would shift product features to a future release and claim success. In other cases, the specifications would be changed to make the test results pass, or products would be released with known defects. In one notable example, the project manger was instructed to falsify paperwork to certify that the project met all of its milestones and was managed by the required process. However, as the project literature suggests, most projects fail, and this was also found among the participants who were solicited for participation in the current study. For their projects, three types of conflict (unexposed, unexplored, and unresolved) were found to represent significant project issues and were implicated directly or indirectly in many of the project failures.

\section{Limitations}

The present study attempted to illustrate the role of conflict as a precursor to the failure of high technology projects. The findings demonstrated how conflict occurred in three conceptual types and how its trajectory was perceived to differentiate successful and unsuccessful projects across a wide variety of organizations and technologies. However, as with any study, a number of limitations must be considered.

Perhaps the primary limitation attributed to qualitative studies is that of generalization. Because the participants reported on their own experiences, which were heavily embedded in their particular environments, these precise situations are unlikely to occur in other high 
technology projects and in other organizations. Consequently, the specific behaviors and actions that were associated with successful and unsuccessful projects in the present study may have little applicability to other similar projects. However, these findings remain valuable for identifying the underlying themes, which represent communication principles applicable across projects and environments. This conclusion is supported by the findings in the current study which represent a wide variety of high technology projects and organizations.

A second limitation is that while the three types of conflict found in the current study were most prevalent and consistent conflict precursors, they may not be exhaustive categories. Other types of conflict may occur that are conceptually distinct from these and may provide important insights to the successful completion for some projects or particular participants. Future research may identify and describe such categories.

A third limitation is that while sensemaking provides a robust explanation for how disparate meanings are constructed by individuals in their projects, it has less strength in explaining how these meanings become resilient and resistant to change when individuals gain new and more salient cues to evaluate their environments. Some recent research has begun to explore this topic and suggested that

People instantly identify with their arguments and positions, that these arguments and positions become part of their self-concept, and that any threat or opposition to these arguments thus represents a threat to the self. This ego-threat then triggers hostility, competitive cognitions, and exceedingly difficult and tenacious exchanges. In other words, we suggest that conflict in and by itself produces ego-threat and is, therefore, difficult to manage and is bound to escalate. (De Dreu \& Van Knippenberg, 2005, p. 345)

In this way, sensemaking in its traditional incarnations may be too narrow to adequately map these deeper affective and self-concept motives to actual conflict behaviors.

Finally, while a wide range of organizations and project types were represented, the current study may not capture the representative communication behaviors of team members in other types of organizations. In fact, it is presumed likely that other project types (e.g. construction projects) are systematically different and may operate under vastly different 
organizational norms and assumptions. In addition, when projects take on a repetitive form, expectations and understandings may routinely be carried in the prior experiences of the participants, reducing the need for explicit communication to occur at all and altering the development and trajectory of conflict.

\section{Implications}

In exploring the nature of conflict in high technology projects and looking for the influences of context, the current study found that projects were substantially different in organizations by virtue of how projects operate and how organizations view the criteria for success. Such differences may have important implications for future research in this area. Also, the finding that conflict, in its various forms, can operate as precursor to project outcomes, suggests that the potential may exist to assess conflict for its indicative properties in foreshadowing project outcomes. These implications are discussed separately in more detail next. Project Differences

An overarching implication that emerges from the current study is that not all high technology projects are alike and their differences may be confounding the results of numerous studies which attempt to find common factors in project behaviors or outcomes. For example, the participants in the current study represented two major types of projects. Development projects were those that often were designed around fixed product specifications where the team members had little or no contact with their customers. These teams primarily consisted of technical members and often conducted their work in secret to avoid competitors discovering their potential products. The second type was IT projects, which were those that were often designed for a particular customer to implement a customized product at the customer site. In these projects, numerous customer representatives were actually part of the team and often jointly responsible for the success of the project. Such projects had a different look and feel as frequent meetings allowed for ongoing communication and more opportunities for differences to be discovered. 
Another difference in projects was the unexpected finding that organizations varied significantly in their orientation toward project success. As described earlier, organizations could be viewed as supporting a (1) power perspective, (2) functional perspective, or (3) relational perspective; which appeared to have a significant impact on how the team members interacted and performed their project tasks. Taken together, these organizational differences created a very different environment for the participants to operate within. Future researchers, especially those who use quantitative methods may benefit from controlling for such project differences.

\section{Conflict Assessment}

The current findings identifying specific conflict types and their consequences on project outcomes may provide an entry for those who are interested in identifying and assessing important project conflict prior to their effects on project outcomes. That is, that conflict assessment could be used to identify important differences and provide early warning about projects in real time, with the potential to avoid costly failures. While very few empirical studies have investigated early warning signals in projects, researchers have suggested their value in predicting project failure. Following Sanchez and Perez (2004)

Early warning signals do not necessarily analyze what has already taken place. They can be used to hypothesize or predict what may happen in the near future of project development. The early warnings are manifested in many ways (verbally, non-verbally, in writing, as events), with sources being people, companies, documents, and situations (p. 12).

This paradigm for conflict creates a context specific assessment challenge for those who seek to evaluate and intervene in ongoing projects. Traditional quantitative measures, by their nature, are at least incomplete and likely ineffective instruments to assess conflict primarily due to their ignorance of context specific factors and their general inability to deal with multiple meanings for each issue of significance. I disagree on this point with Sanchez and Perez (2004) who recommend a switch from qualitative to quantitative assessment metrics as a project moves through its course. They indicate that qualitative methods are superior in the early project stages because quantitative data are typically not available. However, they undervalue the nature of 
qualitative data in providing access to important sensemaking cues of the participants and how those cues result in meanings that become resistant to change as the project unfolds. This position should not be interpreted as eschewing the value of quantitative data as an efficient mechanism to identify important issues. I simply argue that assessing conflict from the disparate perspectives of the participants provides a valuable qualitative contribution by surfacing important early warning indicators that would very likely not be available quantitatively. How then could the sources of conflict described in the present study actually be uncovered?

One approach that may find promise is the recent work on narrative mediation where conflict has been identified and assessed through a quasi-therapeutic process (Winslade \& Monk, 2001). Here, trained practitioners are invited to intervene to resolve conflicts between individuals. The assessment and resolution processes are communication-based and position the clients as experts in their own experiences who can reveal their own reasons for their beliefs and actions. Such an approach is consistent with Schein's (1987) work on process consulting where the consultant acts as a facilitator for the client who retains ownership of the problem. In both cases, the primary role for the outside expert is to understand the underlying processes that lead to the problem rather than to suggest solutions. These are essentially explorations of sensemaking, which, for a trained listener, can provide important insights about the current situation and the trajectory of the efforts. However, this more subjective approach can still benefit from research that could offer added structure to an assessment process and a consistent language to understand and communicate the findings. Clearly, the value of such work would be found from intervening in projects early enough the change their course.

Recent research has supported the conjecture that active intervention of project conflicts in high technology projects can be a factor in project recovery. Ivory and Alderman (2005) argue that project objectives are inherently contradictory in nature and, therefore, local intervention should be seen as a normal project management activity. Given the findings in this study that project complexity and success criteria are both problematic and dynamic, the difficulty of such 
intervention approaches should not be underestimated. However, effective assessment and intervention could yield significant benefits by reducing project failures as future research projects investigate and develop appropriate tools for this purpose. This is consistent with the results of the current study and raises an important question. That is, if unexplored, unexposed, and unresolved conflicts are confirmed to be robust precursors to project failure, then how well can an effective assessment of the project environment reveal these factors and, consequently, the likelihood of project failure?

In response to this question, traditional assessment techniques that rely on quantitative measures would be likely be problematic by their very nature. That is, statistically validated items which are generated across a wide variety of contexts and conditions would eliminate the precise localized information that may be most significant for a particular project. This would be similar to drawing specific conclusions from the multiple case study approach frequently used in business studies. Theory building through case studies has been criticized by Dyer and Wilkins (1991) quoting Pentland (1999) as an approach that:

sacrifices context and deep structures - in a sense, ruining the story - in favor of building constructs that could be used in traditional variance models . . . In the same way that a survey contains indication for the underlying constructs in a variance theory, narrative text contains indicators for an underlying process theory (p. 711).

Thus, it is not so much that case studies in themselves are a poor source of information rather the methodological focus on reduction presumes that context is superfluous to underlying variables and works to homogenize the meanings ascribed for experiences across participants. In this way, the quantitative treatment of case studies removes important elements such as process and context which may provide important insights for assessment. Therefore, the development of an effective assessment tool for evaluating the significance of conflicts in a project would likely have to retain much of the contextual and processual elements that are implicated in the project outcome. In addition, different strategies may be required for each of the three different underlying issues with communication. For example, in unexposed conflict, the assessment focus might be directed 
toward discovery of alternate meanings and involve extensive access to customer and the documentation of organizational resources. For unexplored conflicts, the role of anonymous informants may be particularly valuable, especially surfacing voices that would otherwise remain silenced. This type of conflict may also be exposed by surfacing organizational contradictions that otherwise protect hidden sources of power. Finally, for unresolved conflicts, the assessment strategy may turn to the practice of negotiation to evaluate how effective the participants are at holding difficult conversations and using problem solving techniques that approach Paretoefficient solutions. In sum, while a qualitative assessment program is proposed to identify the context-specific forms of conflict described in this study, its development and application should be expected to be in conflict with those who advocate quantitative scale measures as more 'objective’.

Viewing Conflict More Broadly

Turning to the historical view of conflict, clearly the notion of conflict styles (Thomas \& Kilmann, 1974) still dominates much of the management literature and has influenced the training of project managers and team members. The five styles (competing, collaborating, avoiding, accommodating, and compromising) are simple to understand and have demonstrated utility for problem solving in many situations. However, for the current study, there was little support for specific styles being preferred or more frequently used by the stakeholders. In some cases, these terms would occur in discussion, but they were applied more to specific situations than to descriptions of a particular "style" of preferred behavior. For example, in a discussion of compromising on resources, a participant indicated that she had to compromise in that one situation because it was the most effective solution. In other cases, she might compete for the same resources.

Another traditional view of conflict is that individuals choose different conflict behaviors based on what is deemed most effective and appropriate for the situation (Canary \& Spitzberg, 1987). From this perspective a conflict competent individual may adopt integrative, distributive, 
or avoidant behaviors based on the specific event. As described by the previous example, this competence theory was supported more broadly as the participants (especially project managers) were highly adaptive to their environment. However, as a theoretical explanation for conflict behavior in the project environment, this explanation is limited by its failure to incorporate how the participants may have different meanings for their experiences and not perceive conflict in the same way. In addition, the influence of power or perceived power on behavior is not explored in this model. Therefore, it is not surprising that the conflict-related data from this study of high technology projects resulted in a multi-faceted structure for project conflict.

In contrast to the traditional theories of conflict, the three types of conflict described in the current study emerged from the participants own experiences in their high technology projects. While conceptually distinct, these three types of conflict (unexposed, unexplored, and unresolved) often occurred together and frequently mutated from one form to another. The theoretical significance of this data centers on how each type of conflict is most characteristic of certain project activities and occurred in concert with a specific form of sensemaking. Further, it explains how conflict may transition from an acute to a chronic form as the members engage in enactment processes, which result in further commitment and justification behaviors. In this way, once project conflicts emerge, they may have an unexpectedly strong propensity to take hold and becoming entrenched in projects with substantial consequences for their outcomes. Future researchers may be interested in exploring more about how this transition process occurs and how entrenched conflict becomes resistant to change. Additionally, researchers may offer solutions for how this conflict trajectory may be interrupted or resolved.

\section{Conclusions}

The present study began with numerous examples of high technology project failures and documented their increasingly negative impact on a wide variety of organizations and stakeholders. Because project-based organizations are among the largest in the global economy (Taylor \& Levitt, 2004), and because most continue to fail (Collyer, 2000; Pelod, 2000), the 
current study has continued the line of research that has been directed toward addressing these issues. However, many of the prior studies have been designed to provide generalized recommendations that would be universally applicable to all projects. The numerous lists and heuristics that have been developed provide many important insights into project problems but their generality (e.g. better management, improved communication, etc.) often makes them lack significant utility, especially when applied to the unique challenges of specific situations and unique environments.

In contrast, the present study used a qualitative approach consisting of detailed interviews and participant observation techniques to assess actual project experiences of the participants from their own perspectives. In that regard, the study investigated how conflict may emerge and become problematic in specific projects and generate enduring influences in their outcomes. The rationale for this approach follows from the research finding cited earlier that most project failures are organizational and communication-based rather than technical or process-oriented.

By taking a constructionist interpretative perspective toward conflict, the current study problematized its underlying components and demonstrated how conflict exists in multiple types, each with the potential to impact project outcomes. In doing so, it has challenged the static presumption of several important project concepts. For example, it has demonstrated that "success" is often an ongoing accomplishment negotiated by the stakeholders and emergent over the course of a project. It has surfaced how power is broadly used to alter communication and the resulting behaviors among team members. Also, it has emphasized the contextual relevance of project activities by demonstrating how environmental influences are not incidental, but rather fundamental to how individuals make project choices and make sense of those choices.

Overall, conflict has been explored as a multi-faceted construct with the propensity to become enduring through the commitment and justification principles of sensemaking. This more complex viewing of conflict has called for a theoretical explanation of how conflict can not just emerge but also become enduring in projects creating the potential for significant 
consequences in project outcomes. Classic sensemaking theory (Weick, 1995) provided the framework for this explanation, especially with its emphasis on enactment and justification processes. This connection between sensemaking and conflict is important because it suggests that significant differences in ongoing meaning systems can be made observable through the existence and expression of conflict. Because of this, opportunities exist for developing more robust models for the trajectory of conflict in project teams along with methods for the assessment of enduring conflict and its potential resolution through effective intervention. 


\section{NOTES}

${ }^{1}$ The PMBOK is a registered trademark of the Project Management Institute. It is an acronym for the Project Management Body of Knowledge and also a formal ANSI standard (\# PMI 99-0012004). With the 2005 version, the document has been referred to as a "guide" to indicate that the body of knowledge about project management is broader than can be contained in this document.

${ }^{2}$ In contrast to the standards set forth in the PMBOK, the European Community and other individual countries have adopted broader standards for project management that are coordinated through the International Project Management Association (IPMA). Rather than following PMI's narrow focus on time, budget, and scope; the IAPM includes a "wider view of the discipline, addressing both the context of project management and the technological, commercial, and general management issues, which it believes are important to successfully accomplishing projects” (P. W. G. Morris, 2001, p. 22).

${ }^{3}$ The concept of "earned value" in large projects has become codified by the American National Standards Institute (ANSI) as ANSI standard 748 and the Electronic Industries Alliance (EIA) as standard 748-A. This standard calls for a number of practices to be performed such as objectively measuring accomplishments and reporting significant variations from the original plan.

\footnotetext{
${ }^{4}$ Since the PMBOK only provides a general structure for best practices, many of the specific processes that were identified as "best practices" were those defined and enforced by the participants’ own organizations.
} 


\section{APPENDIX A}

Interview Guide

(Initial demographic questions to qualify individuals for the study will be provided prior to the interview.)

1. Tell me about your role working as a team member or a project manager on one or more high technology projects.

1A. Follow-up probe to clarify the various roles and project that the subject participated in.

2. As a (team member or project manager) tell me about a critical incident that either caused a project to fail or put it at serious risk of failing?

2A. Follow-up probe to clarify details of the critical incident.

3. When did the project first start to fail?

3A. Follow-up probe.

4. Describe someone else on the project who had a different understanding of the project status.

4A. Follow-up probe. (Ask about additional examples including manager and members.)

5. What actions did you take based on your beliefs that the project was not “on-track?”

5A. Follow-up probe.

6. How did those actions affect the outcome of the project?

6A. Follow-up probe.

7. What were the differences between the "actual" and "stated" project goals?

7A. Follow-up probe.

8. In what ways were you encouraged to voice your concerns about the problems?

8A. Follow-up probe. (Ask about how provided the encouragement.)

9. What would have happened if you voiced your concerns?

9A. Follow-up probe.

10. In what ways were you encouraged to keep your project concerns private?

10A. Follow-up probe. (Ask about how provided the encouragement.) 
11. Tell me more about the conflict that existed on the project team?

12. What caused the critical incident?

12A. Follow up probe to assess the team environment prior to the critical incident.

13. What were the formal stages or phases of the project?

14. Tell me about the formal criteria for success at each stage?

15. Give me an example of something that was required for the project to actually be successful even though it was not listed on the formal requirements?

16. Give me an example of a formal requirement that was not very important?

17. Is there any other information that you think might be helpful to understand more about how team members might have different stories about project than the official story? 


\section{APPENDIX B}

\section{Consent Form}

Project Title: Conflict as a precursor to critical incidents in high technology projects

Researcher: William Reed is a doctoral candidate in the Department of Communication at the University of Missouri-Columbia.

Advisor: $\quad$ Michael Kramer is the chair of the Department of Communication at the University of Missouri-Columbia.

Purpose: $\quad$ I will be conducting a study using interviews to understand conflict in organizational projects

Time: $\quad$ The interview should take from $1 / 2$ to 2 hours depending on how much you choose to participate and on what you have to say. Interviews will be audiotaped. A few weeks after the initial interview, you may be contacted by phone to arrange a final brief meeting of up to 30 minutes, to review and verify the researcher's interpretation of your original comments.

Voluntary: $\quad$ Your participation is voluntary. You may quit at any time and you may refuse to answer any question.

Risk: $\quad$ There is minimal risk involved with the study. There is no more risk than you would experience in your daily interactions.

Benefits: $\quad$ The results of this study may help organizational projects be more successful.

Confidential: Neither your identity nor the identity of the organization will be revealed in either transcripts, written documents, or verbal presentations of the data.

The following steps will be taken to protect your identity and confidentiality.

1. Consent forms will be separated from the data

2. Personal identifying information will be eliminated from the transcripts and any reporting of the data

3. You can refuse to answer any question asked.

4. Audiotapes will be kept in locked cabinet, although they must be saved for 3 years, by federal regulation.

Contact: If you have any questions, feel free to contact the study advisor, Michael Kramer at 882-xxxx. You may also email him at: kramerm@missouri.edu

Questions: If you have any questions about your rights, contact Campus IRB: Office of Research 483 McReynolds Hall

Columbia MO 65211

(573) $\mathrm{xxx}-\mathrm{xxxx}$

Thank you for your participation

William A. Reed

Doctoral candidate

Signing this consent indicates that you understand and agree to the conditions mentioned above.

Signature

Date 


\section{REFERENCES}

Anderson, J. R. (1990). Cognitive psychology and its implications. New York: W. H. Freeman.

Avots, I. (1984). Information systems for matrix organizations. In D. Cleland (Ed.), Matrix management systems handbook. New York: Van Nostrand Reinhold.

Axley, S. (1984). Managerial and organizational communication in terms of the conduit metaphor. Academy of Management Review, 9, 428-437.

Baba, M., Falkenburg, D., \& Hill, D. (1996). Technology management and American culture: Implications for business process reengineering. Research Technology Management, 39, 44-54.

Baron, R. A. (1991). Positive effects of conflict. Employee Responsibilities and Rights Journal, 4, 25-36.

Baxter, L. A. (1980). Conflict management: An episodic approach. Small Group Behavior, 13, 23-42.

Belassi, W., \& Tukel, O. (1996). A new framework for determining critical success/failure factors in projects. International Journal of Project Management, 14, 141-151.

Berger, P., \& Luckmann, T. (1966). The social construction of reality: A treatise in the sociology of knowledge. New York: Doubleday.

Berkun, S. (2005). The art of project management. Cambridge: O'Reilly.

Bernstein, R. J. (1983). Beyond objectivism and relativism: Science, hermeneutics, and praxis. Philadelphia: University of Pennsylvania Press.

Blake, R. R., \& Mouton, J. S. (1964). The managerial grid. Houston: Gulf.

Blake, R. R., \& Mouton, J. S. (1981). Management by grid principles or situationalism: Which? Group and Organization Studies, 6, 439-455.

Blake, R. R., Shepard, H., \& Mouton, J. S. (1964). Managing intergroup conflict in industry. Houston: Gulf. 
Boje, D. M. (1991). The storytelling organization: A study of story performance in an officesupply firm. Administrative Science Quarterly, 36, 106-126.

Bonacich, P. (1970). Putting the dilemma back into Prisoners' dilemma. Journal of Conflict Resolution, 14, 379-387.

Bormann, E. G. (1985). Symbolic convergence theory: A communication formulation. Journal of Communication, Autumn, 128-138.

Bormann, E. G. (1990). Small group communication (3rd ed.). New York: Harper \& Row.

Boster, F. J., \& Levine, T. R. (1988). Individual differences and compliance gaining message selection: The effects of verbal aggressiveness, argumentativeness, dogmatism, and negativism. Communication Research Reports, 5, 114-119.

Boster, F. J., Levine, T. R., \& Kazoleas, D. (1993). The impact of argumentativeness and verbal aggressiveness on strategic diversity and persistence in compliance-gaining behavior. Communication Quarterly, 41, 405-414.

Bowers, K. S. (1973). Situationism in psychology: An analysis and critique. Psychological Review, 80, 307-336.

Brass, D. J., \& Burkhardt, M. E. (1993). Potential power and power use: An investigation of structure and behavior. Academy of Management Journal, 36, 441-470.

Bredillet, C. N. (2004). Understanding the very nature of project management: A praxiological approach. In D. P. Slevin, D. I. Cleland \& J. K. Pinto (Eds.), Innovations: Project management research 2004 (pp. 3-22). Newtown Square, PA: Project Management Institute.

Brew, F. P., \& Cairns, D. R. (2004). Styles of managing interpersonal workplace conflicts in relation to status and face concern: A study with Anglos and Chinese. International Journal of Conflict Management, 15, 27-56.

Briner, W., Geddes, M., \& Hastings, C. (1990). Project leadership. Aldershot: Glower.

Burr, V. (1995). An introduction to social constructionism. New York: Routledge. 
Burrell, N. A., Buzzanell, P. M., \& McMillan, J. J. (1992). Feminine tensions in conflict situations as revealed by metaphoric analyses. Management Communication Quarterly, 6, 115-149.

Butler, A. G. (1973). Project management: A study in conflict. Academy of Management Journal, $16,84-101$.

Cai, D. A., \& Fink, E. L. (2002). Conflict style differences between individualists and collectivists. Communication Monographs, 69, 67-87.

Canary, D. J., Cupach, W. R., \& Serpe, R. T. (2001). A competence based approach to examining interpersonal conflict: Test of a longitudinal model. Communication Research, 28, 79104.

Canary, D. J., \& Spitzberg, B. H. (1987). Appropriateness and effectiveness perceptions of conflict strategies. Human Communication Research, 14, 93-118.

Canary, D. J., \& Spitzberg, B. H. (1989). A model of the perceived competence of conflict strategies. Human Communication Research, 15, 630-649.

Canary, D. J., \& Spitzberg, B. H. (1990). Attribution biases and associations between conflict strategies and competence outcomes. Communication Monographs, 57, 139-151.

Case, R. H., \& Shane, S. (1998). Fostering risk taking in research and development: The importance of a projects terminal value. Decision Science, 29, 765-783.

Cetina, K. D. K. (1981). The manufacture of knowledge: An essay on the constructivist and contextual nature of science. Oxford: Pergamon.

Cicmil, S. (1999). An insight into management of organizational change projects. Journal of Workplace Learning, 11, 5-15.

Cleland, D. (1968). The deliberate conflict. Business Horizons, 11, 78-80.

Cleland, D. (1974). Project management in industry: An assessment. Project management Quarterly, 5, 19-21.

Collyer, M. (2000). Communication - The route to successful change management: Lessons from the Guiness Integrated Business Programme. Supply Chain Management: An International Journal, 5, 554-570. 
Conrad, C. (1991). Communication in conflict: Style-strategy relationships. Communication Monographs, 58, 135-155.

Cooke-Davies, T. (2002). The real success factors on projects. International Journal of Project Management, 20, 185-190.

Cooley, C. H. (1964). Human nature and the social order. New York: Schocken.

Coser, L. A. (1956). The functions of social conflict. New York: Macmillan.

Coser, L. A. (1967). Continuities in the study of social conflict. New York: Free Press.

Cosier, R. A., Dalton, D. R., \& Taylor, L. A. (1991). Positive effects of cognitive conflict and employee voice. Employee Responsibilities and Rights Journal, 4, 7-11.

Creswell, J. W. (1997). Qualitative inquiry and research design: Choosing among five traditions. Thousand Oaks: Sage.

Cronen, V. E., Pearce, W. B., \& Xi, C. (1990). The 'meaning' in the CMM analysis of communication: A comparison of two traditions. Research on Language and Social Interaction, 23, 1-40.

Darling, J. R., \& Brownlee, L. J. (1984). Conflict management in the academic institution. Texas Tech Journal of Education, 11, 243-257.

Davis, L. E., \& Taylor, J. C. (1976). Technology organization and job structure. In R. Dubin (Ed.), Handbook of work organization and society. Skokie, IL: Rand-McNally.

Davis, S. M., \& Lawrence, P. R. (1977). Matrix. Reading, MA: Addison-Wesley.

De Dreu, C. K. W., \& Van Knippenberg, D. (2005). A possessive self as a barrier to conflict resolution: Effects of mere ownership, process accountability, and self-concept clarity on competitive cognitions and behavior. Journal of Personality and Social Psychology, 89, 345-357.

Deal, T., \& Kennedy, A. (1982). Corporate culture: Rites and rituals of organizational life. Reading, MA: Addison-Wesley. 
Denis, H. (1986). Matrix structures, quality of life, and engineering productivity. IEEE Transactions on Engineering Management, 33, 148-156.

Deutsch, M. (1973). Conflicts: productive and destructive. In F. E. Jandt (Ed.), Conflict resolution through communication. New York: Harper \& Row.

Dhillon, G. (2003). Dimensions of power and IS implementation. Information and Management, 41, 635-644.

Donohue, W., \& Colt, R. (1992). Managing interpersonal conflict. Newbury Park, CA: Sage.

Donohue, W. A. (1981). Analyzing negotiation tactics: Development of a negotiation interact system. Human Communication Research, 7, 273-287.

Dozier, J. B., \& Miceli, M. P. (1985). Potential predictors of whistle-blowing: A prosocial behavior perspective. Academy of Management Review, 10, 823-836.

Dyer, W. G. J., \& Wilkins, A. (1991). Better stories, not better constructs, to generate better theory. A rejoinder to Eisenhart. Academy of Management Review, 16, 613-620.

Evans, D. R., Hearn, M. T., Uhlemann, M. R., \& Ivey, A. E. (1998). Essential interviewing: A programmed approach to effective communication (5th ed.). Pacific Grove, CA: BrooksCole.

Festinger, L. (1957). A theory of cognitive dissonance. Evanston, Ill.: Peterson Row.

Field, T. (1997). When bad things happen to good projects. CIO, 11, 54.

Fink, C. F. (1968). Some conceptual difficulties in the theory of social conflict. Conflict Resolution, 12, 412-460.

Fisher, R., Ury, W., \& Patton, B. (1991). Getting to yes: Negotiating agreement without giving in (2nd ed.). New York: Penguin.

Folger, J. P., Poole, M. S., \& Stutman, R. (1993). Working through conflict: A communication perspective. Glenview, Il: Scott, Foresman and Co.

Ford, R. C., \& Randolph, A. W. (1992). Cross functional structures: A review of integration of matrix organization and project management. Journal of Management, 18, 267-294. 
Foucault, M. (1972). The archeology of knowledge. New York: Pantheon.

Foucault, M. (1980). Power/knowledge: Selected interviews and other writings, 1972-1977. New York: Pantheon Books.

Frame, J. D. (1995). Managing projects in organizations: How to make the best use of time, techniques, and people. San Francisco: Jossey-Bass.

Freedman, J., \& Combs, G. (1996). Narrative Therapy: The social construction of preferred realities. New York: W. W. Norton.

Garcia, S. (2005). How standards enable adoption of project management practice. IEEE Software, September-October, 22-29.

Gardner, D. J. (2000). How to avoid IT project failures. Consulting to Management, 11, 21-23.

Gertz, C. (1973). The interpretation of cultures. New York: Basic Books.

Gibbs, W. W. (1994). Software's chronic crisis. Scientific American, 271(September), 86-95.

Gibson, C. B. (1997). Do you hear what I hear? A framework for reconciling intercultural communication difficulties arising from cognitive styles and cultural values. In P. C. Earley \& M. Erez (Eds.), New Perspectives on industrial/organizational psychology (pp. 335-361). San Francisco: New Lexington.

Giddens, A. (1984). The constitution of society. Cambridge: Polity.

Glaser, J. (2005). More on management's role in IT project failures. Healthcare Financial Management, January, 82-84.

Goffman, E. (1959). The presentation of self in everyday life. New York: Overlook.

Goodman, R. A. (1967). Ambiguous authority definition in project management. Academy of Management Journal, 10, 395-407.

Gray, R. J. (2001). Organizational climate and project success. International Journal of Project Management, 19, 103-109. 
Hall, J. (1969). Conflict management survey. Conroe, Tx: Teleometrics.

Hall, J. (1986). Conflict management survey: A survey of one's characteristic reaction to and handling of conflicts between himself and others. Conroe, TX: Teleometrics.

Hallgren, M., \& Maaninen-Olsson, E. (2005). Deviations, ambiguity, and uncertainty in a projectintensive organization. Project Management Journal, 36, 17-26.

Hanssen, C. D., \& Kahnweiler, W. M. (1997). Executive managers: Cultural expectations through stories about work. Journal of Applied Management Studies, 6, 117-138.

Hatch, M. J. (1993). The dynamics of organizational culture. Academy of Management Review, 18, 657-693.

Hawes, L. C., \& Smith, D. H. (1973). A critique of assumptions underlying the study of communication in conflict. Quarterly Journal of Speech, 59, 423-435.

Heracleous, L. (2000). The role of strategy implementation in organization development. Organization Development Journal, 18, 75-86.

Herrmann, M. G., \& Kogan, N. (1977). Effects of negotiators' personalities on negotiating behavior. In D. Druckman (Ed.), Negotiations (pp. 247-274). Beverly Hills: Sage.

Hocker, J. L., \& Wilmot, W. W. (1995). Interpersonal conflict (4th ed.). Dubuque, Ia: Brown \& Benchmark.

Hofestede, G. (1980). Cultures consequences: International differences in work related values. Beverly Hills: Sage.

Hofestede, G. (1997). Cultures and organizations: Software of the mind. New York: McGrawHill.

Holstein, J. A., \& Gubrium, J. F. (1995). The active interview. Thousand Oaks: Sage.

Infante, D. A. (1981). Trait argumentativeness as a predictor of communicative behavior in situations requiring argument. Central States Speech Journal, 32, 265-272.

Infante, D. A., \& Rancer, A. S. (1982). A conceptualization and measure of argumentativeness. Journal of Personality Assessment, 46, 72-80. 
Infante, D. A., \& Wigley, C. J. (1986). Verbal aggressiveness: An interpersonal model and measure. Communication Monographs, 53, 61-69.

Ives, M. (2005). Identifying the contextual elements of project management within organizations and their impact on project success. Project Management Journal, 36, 37-50.

Ivory, C., \& Alderman, N. (2005). Can project management learn anything from studies of failure in complex systems? Project Management Journal, 36, 5-16.

Jaafari, A. (2003). Project management in the age of complexity and change. Project Management Journal, 34, 47-57.

Jaafari, A., Doloi, H., \& Gunaratnam, D. (2004). Life cycle project management: A platform for strategic project management. In D. P. Slevin, D. I. Cleland \& J. K. Pinto (Eds.), Innovations: Project management research 2004 (pp. 141-160). Newtown Square, PA: Project Management Institute.

Jehn, K. A. (1995). A multimethod examination of the benefits and detriments of intragroup conflict. Administrative Science Quarterly, 40, 256-282.

Jerkovsky, W. (1983). Functional management in matrix organizations. IEEE Transactions on Engineering Management, 30, 89-97.

Jordan, J. (1990). Courage in connection: Conflict, compassion, creativity. Work In Progress, 112.

Joyce, W. F. (1986). Matrix organization: A social experiment. Academy of Management Journal, 29, 536-561.

Kant, I. (1929). The critique of pure reason (N. K. Smith, Trans.). London: McMillan.

Katz, R., \& Allen, T. J. (1985). Project performance and the locus of influence in the R \& D matrix. Academy of Management Journal, 28, 67-87.

Keil, M. (1995). Pulling the plug: Software project management and the problem of escalation. MIS Quarterly, December, 421-447.

Keil, M., Cule, P. E., Lyytinen, K., \& Schmidt, R. C. (1998). A framework for identifying software project risks. Communications of the ACM, 41, 76-83. 
Keil, M., \& Robey, D. (1999). Turning around troubled software projects: An exploratory study of the deescalation of commitment to failing courses of action. Journal of Management Information Systems, 15, 63-87.

Keil, M., \& Robey, D. (2001). Blowing the whistle on troubled software projects. Association for Computing Machinery, 44, 87-93.

Kendra, K., \& Taplin, L. J. (2004). Project success: A cultural framework. Project Management Journal, April, 30-45.

Kenny, J. (2003). Effective project management for strategic innovation and change in an organizational context. Project Management Journal, 34, 43-53.

Kilmann, R. H., \& Thomas, K. W. (1975). Interpersonal conflict-handling behavior as a reflection of Jungian personality dimensions. Psychological Reports, 37, 971-980.

Kinsella, W. J. (1999). Discourse, power, and knowledge in the management of "big science". Management Communication Quarterly, 13, 171-208.

Kirchmeyer, C., \& Cohen, A. (1992). Multicultural groups: Their performance and reactions with constructive conflict. Group and Organization Management, 17, 153-170.

Kirchof, N. S., \& Adams, J. R. (1989). Conflict management for program managers. Pennsylvania: Project Management Institute.

Kleim, R. L., \& Landin, I. S. (1992). The people side of projects. Aldershot: Glower.

Knapp, M. L., Putnam, L., \& Davis, L. J. (1988). Measuring interpersonal conflict in organizations: Where do we go from here. Management Communication Quarterly, 1, 414-429.

Knight, K. (1976). Matrix organization: A review. Journal of Management Studies, 17, 111-130.

Kolodny, H. F. (1979). Evolution to a matrix organization. Academy of Management, 23, 29-33.

KPMG. (1995). Runaway projects: Causes and effects. Software World, 26, 3-5.

Kuhn, T. (1996). The structure of scientific revolutions (3rd ed.). Chicago: University of Chicago Press. 
Kuhn, T. (2000). The road since structure. Chicago: University of Chicago Press.

Kvale, S. (1996). Interviews: An introduction to qualitative research interviewing. Thousand Oaks: Sage.

Lakey, S. G., \& Canary, D. J. (2002). Actor-goal achievement and sensitivity to partner as critical success factors in understanding interpersonal communication competence. Communication Monographs, 69, 217-235.

Larson, E. W., \& Gobeli, D. H. (1987). Matrix management: Contradictions and insights. California Management Review, 29, 126-138.

Leung, K. (1988). Some determinants of conflict avoidance. Journal of Cross-Cultural Psychology, 19, 125-136.

Leung, K., Koch, P. M., \& Lu, L. (2002). A dualistic model of harmony and its implications for conflict management in Asia. Asia Pacific Journal of Management, 19, 201-220.

Levine, T. R., \& Boster, F. J. (1996). The impact of self and others' argumentativeness on talk about controversial issues. Communication Quarterly, 44, 345-358.

Lindlof, T. R., \& Taylor, B. C. (2002). Qualitative Communication Research Measures (2nd ed.). Thousand Oaks: Sage.

Litterer, J. (1966). Conflict in organizations. Academy of Management Journal, 9, 178-186.

Littlejohn, S. W. (1983). Theories of human communication (2nd ed.). Belmont: Wadsworth.

Lyytinen, K., \& Robey, D. (1999). Learning failures in information systems development. Information Systems Journal, 9, 85-101.

Martin, A., \& Chan, M. (1996). Information systems project redefinition in New Zealand: Will we ever learn? Austrailian Computer Journal, 28.

McBride, T., Henderson-Sellers, B., \& Zowghi, D. (2004). Project management capability levels: An empirical study. Paper presented at the Asia-Pacific Engineering Conference, Sydney.

McCray, G. E., Purvis, R. L., \& McCray, C. G. (2002). Project management under uncertainty: The impact of heuristics and biases. Project Management Journal, 33, 49-57. 
Montealegre, R., \& Keil, M. (2000). De-escalating information technology projects: Lessons from the Denver International Airport. MIS Quarterly, 24, 417-447.

Morris, M. W., Williams, K. Y., Leung, K., Larrick, R., Mendoza, D. B., Li, J., et al. (1998). Conflict management styles: Accounting for cross-national differences. Journal of International Business Studies, 29, 729-748.

Morris, P. W. G. (2001). Updating the project management bodies of knowledge. Project Management Journal, 32, 21-30.

Neale, M. A., Huber, V. L., \& Northcraft, G. B. (1987). The framing of negotiations: Contextual versus task frames. Organizational Behavior and Human Decision Processes, 39, 228241.

Nguyen, T. N. (2006). A decision-model for managing software development projects. Information and Management, 43, 63-75.

Nicotera, A. M. (1993). Beyond two dimensions: A grounded theory model of conflict handling behavior. Management Communication Quarterly, 6, 282-306.

Nicotera, A. M. (1995). Thinking about communication and conflict. In A. M. Nicotera (Ed.), Conflict and organizations (pp. 3-15). Albany, NY: State University of New York.

Nicotera, A. M., Rodriguez, A. J., Hall, M., \& Jackson II, R. L. (1995). A history of the study of communication and conflict. In A. M. Nicotera (Ed.), Communication and organizations (pp. 17-41). Albany: State University of New York.

Oltman, P. K., Goodenough, D. R., Witkin, H., Freedman, N., \& Friedman, F. (1975). Psychological differentiation as a factor in conflict resolution. Journal of Personality and Social Psychology, 32, 730-736.

Onyekwere, E. O., Rubin, R. A., \& Infante, D. A. (1991). Interpersonal perception and communication satisfaction as a function of argumentativeness and ego-involvement. Communication Quarterly, 39, 35-47.

Orlikowski, W. J. (1997). The duality of technology in organizations. Organization Science, 3, 398-427.

Owen, W. F. (1984). Interpretive themes in relational communication. Quarterly Journal of Speech, 70, 274-287. 
Oz, E. (1994). When professional standards are lax: The CONFIRM failure and its lessons. Communications of the ACM, 37, 29-36.

Papa, M. J., \& Canary, D. J. (1995). Conflict in organizations: A competence based approach. In A. M. Nicotera (Ed.), Conflict and organizations (pp. 153-179). New York: State University of New York Press.

Pearce, W. B. (1989). Communication and the human condition. Carbondale, IL: Southern Illinois University Press.

Pelod, A. (2000). Politicking for success: The missing skill. Leadership and organization development journal, 21, 20-29.

Pentland, B. T. (1999). Building process theory with narrative: From description to explanation. Academy of Management Review, 24, 711-724.

Perrow, C. (1984). Normal accidents. New York: Basic Books.

Phillips, F. (2002). The distortion of criteria after decision-making. Organizational Behavior and Human Decision Processes, 88, 769-784.

Pierce. (1979). The nature of natural languages. Portland: HAPI Press.

Pinto, J. K., \& Kharbanda, O. P. (1996). How to fail in project management (without really trying). Business Horizons, 39, 45-54.

Pinto, J. K., \& Mantel, S. J. (1990). The causes of project failure. IEEE Transactions on Engineering Management, 37, 269-276.

Pinto, J. K., \& Roughiainen, P. (2001). Building customer-based project organizations. New York: John Wiley \& Sons.

Pinto, J. K., \& Slevin, D. P. (1987). Critical factors in successful project implementation. IEEE Transactions on Engineering Management, 34, 22-27.

PMI. (2005a). A guide to the project management body of knowledge. Newtown Square, PA: Project Management Institute. 
PMI. (2005b). Project management institute. Retrieved June 20, 2005, from http://www.pmi.org/info/default.asp

PMI Standards Committee. (1996). A guide to the project management body of knowledge. Upper Derby, PA: Project Management Institute.

Pondy, L. R. (1966). A systems theory of organizational conflict. Academy of Management Journal, 9, 246-256.

Presnell, M. (1994). Postmodern ethnography: From representing the other to co-producing a text. In K. Carter \& M. Presnell (Eds.), Interpretive approaches to interpersonal communication (pp. 11-43). New York: State University of New York Press.

Pruitt, D. (1983). Strategic choice in negotiation. American Behavioral Scientist, 27, 167-194.

Pruitt, D., \& Rubin, J. Z. (1986). Social conflict: Escalation, stalemate, settlement. New York: Random House.

Purvis, R. L., McCray, G. E., \& Roberts, T. L. (2002). The impact of management heuristics to IS projects. Paper presented at the 36th Hawaii International Conference on System Sciences, Hawaii.

Putnam, L. L. (1988). Communication and interpersonal conflict in organizations. Management Communication Quarterly, 1, 293-301.

Putnam, L. L. (1995). Formal negotiations: The productive side of organizational conflict. In A. M. Nicotera (Ed.), Conflict and organizations (pp. 183-200). New York: State University of New York Press.

Putnam, L. L., \& Jones, T. S. (1982). Reciprocity in negotiations: An analysis of bargaining interaction. Communication Monographs, 49, 171-191.

Putnam, L. L., \& Poole, M. S. (1987). Conflict and negotiation. In F. M. Jablin, L. L. Putnam, K. H. Roberts \& L. W. Porter (Eds.), Handbook of organizational communication. Beverly Hills: Sage.

Putnam, L. L., \& Stohl, C. (1990). Bona fide groups: A reconceptualization of groups in context. Communication Studies, 25, 248-265. 
Putnam, L. L., \& Wilson, C. E. (1982). Communicative strategies in organizational conflicts: Reliability and validity of a measurement scale. Communication Yearbook, 6, 629-652.

Rahim, M. A. (1983). A measure of styles in handling interpersonal conflict. Academy of Management Journal, 26, 368-376.

Rahim, M. A., \& Bonoma, T. V. (1979). Managing organizational conflict: A model for diagnosis and intervention. Psychological Reports, 44, 1323-1344.

Raisanen, C., \& Linde, A. (2004). Technologizing discourses to standardize projects in multiproject organizations: Hegemony by consensus? Organization, 11, 101-121.

Randolph, W. A., \& Posner, B. Z. (1992). Getting the job done: Managing project teams and task forces for success. Englewood Cliffs, NJ: Prentice-Hall.

Rice, G. E. (1980). On cultural schemata. American Ethnologist, 7, 152-171.

Riessman, C. K. (2001). Analysis of personal narratives. In J. F. Gubrium \& J. A. Holstein (Eds.), Handbook of interview research: Context and method (pp. 695-710). Newbury Park: Sage.

Ring, P. S., \& Rands, G. P. (1989). Sensemaking, understanding, and committing: Emergent interpersonal transaction processes in the evolution of 3M's microgravity research program. In A. H. Van de Ven, H. L. Angle \& M. S. Poole (Eds.), Research on the management of innovation: The Minnesota studies (pp. 337-366). New York: Ballinger.

Rizzo, J. R., House, R. J., \& Lirtzman, S. I. (1970). Role conflict and ambiguity in complex organizations. Administrative Science Quarterly, 15, 150-163.

Roberts, T. L., Cheney, P. H., Sweeney, P. D., \& Hightower, R. T. (2005). The effects of information technology project complexity on group interaction. Journal of Management Information Systems, 21, 223-247.

Ross, L., \& Nisbett, R. E. (1991). The person and the situation. New York: McGraw-Hill.

Ross, R., \& DeWine, S. (1982). Interpersonal conflict: Measurement and validation. Louisville, $\mathrm{KY}$.

Ross, R. G., \& DeWine, S. (1988). Assessing the Ross-DeWine conflict management style (CMMS). Management Communication Quarterly, 1, 389-413. 
Roy, V., \& Aubert, B. (2003). A dream project turns nightmare: How flawless software never got implemented. Annals of Cases on Information Technology, 5, 98-111.

Royer, P. S. (2000). Risk management: The undiscovered dimension of project management. Project Management Journal, 31, 6-13.

Rubin, B. D. (1978). Communication and conflict: A system-theoretic perspective. Quarterly Journal of Speech, 64, 202-210.

Ruble, T. L., \& Thomas, K. W. (1976). Support for a two-dimensional model of conflict behavior. Organizational Behavior and Human Decision Processes, 16, 143-155.

Sanchez, A. M., \& Perez, M. P. (2004). Early warning signals for R \& D projects: An empirical study. Project Management Journal, 35, 11-23.

Sass, C. (1994). On interpersonal competence. In K. Carter \& M. Presnell (Eds.), Interpretive approaches to interpersonal communication (pp. 137-157). New York: State University of New York Press.

Sauer, C., Liu, L., \& Johnston, K. (2001). Where project managers are kings. Project Management Journal, 32, 39-49.

Schein, E. (1987). Process consultation: Volume II Lessons for managers and consultants. Menlo Park: Addison-Wesley.

Schein, E. (1992). Organizational culture and leadership. San Francisco: Jossey Bass.

Schockley-Zalabak, P. (1988). Assessing the Hall Conflict Management Survey. Management Communication Quarterly, 1, 302-320.

Schrank, R. (1990). Tell me a story. A new look at real and artificial memory. New York: MacMillian.

Seidman, I. (1998). Interviewing as qualitative research: A guide for researchers in education and the social sciences. New York: Teachers College Press.

Shannon, C., \& Weaver, W. (1949). The mathematical theory of communication. Urbana, IL: University of Illinois Press. 
Shotter, J. (1990). Knowing of the third kind. Utrecht: University of Utrecht.

Shubik, M. (1970). Game theory, behavior, and the paradox of the Prisoners' dilemma: Three solutions. Journal of Conflict Resolution, 14(181-193).

Simon, H. A. (1992). What is an explanation of behavior? Psychological Science, 3, 150-161.

Sims, D. (1993). Coping with misinformation. Management Decision, 31, 18-21.

Slack, B. D., \& Cook, J. O. (1973). Authoritarian behavior in a conflict situation. Journal of Personality and Social Psychology, 25, 130-136.

Smith, H. J., Keil, M., \& Depledge, G. (2001). Keeping mum as the project goes under: Toward an explanatory model. Journal of Management Information Systems, 18, 189-227.

Spitzberg, B. H. (1983). Communication competence as knowledge, skill, and impression. Communication Education, 32, 323-328.

Spitzberg, B. H., \& Canary, D. J. (1985). Loneliness and relationally competent communication. Journal of Social and Personal Relationships, 2, 387-402.

Spitzberg, B. H., Canary, D. J., \& Cupach, W. R. (1994). A competence-based approach to the study of interpersonal conflict. In D. Cahn (Ed.), Conflict in personal relationships. Hillsdale, NJ: Lawrence Erlbaum.

Standish Group International, I. (1999). CHAOS: A recipe for success: Research report: Ordering information available at www.standishgroup.com.

Starbuck, W. H., \& Milliken, F. J. (1988). Executives' perceptual filters: What they notice and how they make sense. In D. C. Hambrick (Ed.), The executive effect: Concepts and methods for studying top managers (pp. 35-65). Greenwich, CT: JAI.

Steinfatt, T., \& Miller, G. R. (1974). Communication in game theoretic models of conflict. In G. R. Miller \& H. W. Simmons (Eds.), Perspectives on communication in social conflict (pp. 14-71). Englewood Cliffs, NJ: Prentice Hall.

Sternberg, R. J., \& Dobson, D. M. (1987). Resolving interpersonal conflicts: An analysis of stylistic inconsistency. Journal of Personality and Social Psychology, 52, 794-812. 
Sternberg, R. J., \& Soriano, L. J. (1984). Styles of conflict resolution. Journal of Personality and Social Psychology, 47, 115-126.

Stuckenbruck, L. C. (1982). The implementation of program management: The professional's handbook. Boston, MA: Addison-Wesley.

Taylor, J. E., \& Levitt, R. E. (2004). Understanding and managing systemic innovation in projectbased industries. In D. P. Slevin, D. I. Cleland \& J. K. Pinto (Eds.), Innovations: Project management research 2004 (pp. 83-99). Newtown Square, PA: Project Management Institute.

Thamhain, H. J. (2004). Team leadership effectiveness in technology-based project environments. Project Management Journal, 35, 35-46.

Thamhain, H. J., \& Wilemon, D. L. (1975). Conflict management in program life cycles. Sloan Management Review, 16, 31-50.

Thomas, K. W. (1988). The conflict-handling modes: Toward more precise theory. Management Communication Quarterly, 1, 430-436.

Thomas, K. W. (1992). Conflict and conflict management: Reflections and update. Journal of Organizational Behavior, 13, 265-274.

Thomas, K. W., \& Kilmann, K. W. (1977). Developing a forced-choice measure of conflicthandling behavior: The MODE instrument. Educational and Psychological Measurement, 37, 390-395.

Thomas, K. W., \& Kilmann, R. H. (1974). Thomas-Kilmann conflict mode instrument. Tuxedo, NY: Xicom.

Thompson, I., \& Richardson, B. (1996). Strategic and competitive success: Towards a model of the comprehensively competent organization. Management Decision, 34, 5-19.

Thorne, A. (1987). The press of personality: A study of conversations between introverts and extroverts. Journal of Personality and Social Psychology, 53, 718-726.

Ting-Toomey, S. (1988). Intercultural conflicts styles: A face-negotiation theory. In Y. Kim \& W. Gudykunst (Eds.), Theories in intercultural communication (pp. 213-235). Newbury Park: Sage. 
Ting-Toomey, S., \& Kurogi, A. (1998). Facework competence in intercultural conflict: An updated face-negotiation theory. International Journal of Intercultural Relations, 22, 187-225.

Tversky, A., \& Kahneman, D. (1974). Judgment under uncertainty: Heuristics and biases. Science, 185, 1124-1131.

Urli, B., \& Urli, D. (2000). Project management in North America: Stability and concepts. Project Management Journal, 31, 33-43.

van de Vliert, E., Nauta, A., Giebels, E., \& Janssen, O. (1999). Constructive conflict at work. Journal of Organizational Behavior, 20, 475-491.

Volkema, R., Farquhar, H., \& Bergmann, T. (1996). Third party sensemaking in interpersonal conflicts at work: A theoretical framework. Human Relations, 49, 1437-1454.

Wall Jr., V. D., Galanes, G. J., \& Love, S. B. (1987). Small, task-oriented groups: Conflict, conflict management, satisfaction, and decision quality. Small Group Behavior, 18, 3155.

Walton, R., \& McKersie, R. (1965). A behavioral theory of labor negotiations. New York: McGraw-Hill.

Wang, X. (2003). Developing a true sense of professional community: An important matter for PM professionalism. Project Management Journal, 33, 5-11.

Warren, C. A. B. (2001). Qualitative interviewing. In J. F. Gubrium \& J. A. Holstein (Eds.), Handbook of interview research: Context and method (pp. 83-101). Thousand Oaks: Sage.

Watzlawick, P. (1976). How real is real. New York: Random House.

Weick, K. E. (1979). The social psychology of organizing (2nd ed.). New York: McGraw-Hill.

Weick, K. E. (1987). Substitutes for strategy. In D. J. Teece (Ed.), The competitive challenge: Strategies for industrial innovation and renewal (pp. 221-233). Cambridge, MA: Ballinger.

Weick, K. E. (1995). Sensemaking in organizations. Thousand Oaks: Sage. 
Weick, K. E. (2001). Making sense of the organization. Malden: Blackwell.

Weick, K. E., \& Browning, L. (1986). Arguments and narration in organizational communication. Journal of Management, 12, 243-259.

Weinstein, D. (1979). Bureaucratic opposition: Challenging abuses in the workplace. New York: Pergamon Press.

Wellmon, T. A. (1988). Conceptualizing organizational communication competence: A rules perspective. Management Communication Quarterly, 1, 515-534.

Whittaker, B. (1999). Information management and computer security. Bradford, 7, 23-27.

Williams, T. (2005). Assessing and moving on from the dominant project management discourse in the light of project overruns. IEEE Transactions on Engineering Management, 52, 497-508.

Wilmot, W. W. (1987). Dyadic communication (3rd ed.). New York: McGraw-Hill.

Wilson, S. R., \& Waltman, M. (1988). Assessing the Putnam-Wilson organizational conflict instrument. Management Communication Quarterly, 1, 367-388.

Winch, G. M. (2004). Rethinking project management: Project organizations as information processing systems. In D. P. Slevin, D. I. Cleland \& J. K. Pinto (Eds.), Innovations: Project management research 2004 (pp. 41-56). Newtown Square, PA: Project Management Institute.

Winslade, J., \& Monk, G. (2001). Narrative Mediation. San Francisco: Jossey-Bass.

Womack, D. F. (1988a). Assessing the Thomas-Kilmann conflict MODE survey. Management Communication Quarterly, 1, 321-349.

Womack, D. F. (1988b). A review of conflict instruments in organizational settings. Management Communication Quarterly, 1, 437-445.

Wood, J. T., Dendy, L. L., Dordek, E., Germany, M., \& Varallo, S. M. (1994). Dialectic of differences: A thematic analysis of intimates' meanings for differences. In K. Carter \& M. Presnell (Eds.), Interpretive approaches to interpersonal communication (pp. 115-136). New York: State University of New York Press. 
Wood, R. (1986). Taks complexity: Definition of a construct. Organizational Behavior and Human Decision Processes, 37, 60-82.

Wright, C. R., Manning, M. R., Farmer, B., \& Gilbreath, B. (2000). Resourceful sensemaking in product development teams. Organization Studies, 21, 807-825.

Xi, J., Song, X. M., \& Stringfellow, A. (1998). Interfunctional conflict, conflict resolution styles, and new product success: A four culture comparison. Management Science, 44, 192-206. 


\section{VITA}

William Reed received a Bachelors of Science in Electronics Engineering from the University of Texas at Arlington (1976) with an emphasis on software and digital electronics. He received U.S. and international patents for his design and development of electronic security equipment. He has over 20 years of experience in project and departmental management occupying senior management positions in two organizations. After completing additional undergraduate and graduate coursework in management, he completed a Master of Science (2000) in Organizational Communication at the University of North Texas and a Ph.D. at the University of Missouri-Columbia (2006). 